\title{
Diagnostic and therapeutic strategies in renal artery stenosis
}

Citation for published version (APA):

van Onna, M. (2007). Diagnostic and therapeutic strategies in renal artery stenosis. [Doctoral Thesis, Maastricht University]. Datawyse / Universitaire Pers Maastricht. https://doi.org/10.26481/dis.20071130mo

Document status and date:

Published: 01/01/2007

DOI:

10.26481/dis.20071130mo

Document Version:

Publisher's PDF, also known as Version of record

\section{Please check the document version of this publication:}

- A submitted manuscript is the version of the article upon submission and before peer-review. There can be important differences between the submitted version and the official published version of record.

People interested in the research are advised to contact the author for the final version of the publication, or visit the DOI to the publisher's website.

- The final author version and the galley proof are versions of the publication after peer review.

- The final published version features the final layout of the paper including the volume, issue and page numbers.

Link to publication

\footnotetext{
General rights rights.

- You may freely distribute the URL identifying the publication in the public portal. please follow below link for the End User Agreement:

www.umlib.nl/taverne-license

Take down policy

If you believe that this document breaches copyright please contact us at:

repository@maastrichtuniversity.nl

providing details and we will investigate your claim.
}

Copyright and moral rights for the publications made accessible in the public portal are retained by the authors and/or other copyright owners and it is a condition of accessing publications that users recognise and abide by the legal requirements associated with these

- Users may download and print one copy of any publication from the public portal for the purpose of private study or research.

- You may not further distribute the material or use it for any profit-making activity or commercial gain

If the publication is distributed under the terms of Article $25 \mathrm{fa}$ of the Dutch Copyright Act, indicated by the "Taverne" license above, 
Diagnostic and therapeutic strategies in renal artery stenosis 
(C) Marieke van Onna, Maastricht 2007

ISBN: 978-90-5278-679-7

Cover design: Bas Verhoef, Toelgroep

Layout: Tiny Wouters

Production: Datawyse | Universitaire Pers Maastricht

Financial support by the Netherlands Heart Foundation, the Dutch Kidney Foundation, Astellas Pharma B.V., Baxter B.V., MSD and Sanofi-Aventis Netherlands B.V. for the publication of this thesis is gratefully acknowledged. 


\title{
Diagnostic and therapeutic strategies in renal artery stenosis
}

\author{
PROEFSCHRIFT \\ ter verkrijging van de graad van doctor \\ aan de Universiteit Maastricht, \\ op gezag van de Rector Magnificus, Prof. mr. G.P.M.F. Mols, \\ volgens het besluit van het College van Decanen, \\ in het openbaar te verdedigen \\ op vrijdag 30 november 2007 om 14.00 uur
}

door

Marieke van Onna

Geboren op 25 mei 1976 te Sittard

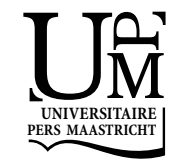




\section{Promotores}

Prof. dr. P.W. de Leeuw

Co-promotor

Dr. A.A. Kroon

Dr. A.J.H.M. Houben

Beoordelingscommissie

Prof. dr. J.M.A. van Engelshoven (voorzitter)

Prof. dr. P.J.E.H.M. Kitslaar

Prof. dr. H.A. Koomans, UMC Utrecht

Prof. dr. P.F. Plouin, Hôpital Européen George Pompidou, Paris, France

Prof. dr. M.H. Prins 
Aan mijn ouders 



\section{Contents}

$\begin{array}{llr}\text { Chapter } 1 & \text { Introduction } & 9\end{array}$

Chapter 2 The predictive value of established clinical clues for the 17 diagnosis of renal artery stenosis in clinical practice

Chapter 3 The additional value of measuring IMT, PWV and ambulatory 33 blood pressure in the diagnosis of renal artery stenosis

Chapter 4 Genetic risk of atherosclerotic renal artery disease: the candidate gene approach in a renal angiography cohort

Chapter 5 Asymmetry of renal blood flow in patients with moderate to severe hypertension

Chapter 6 Functional consequences of asymmetry in renal blood flow in hypertensive patients with unilateral atherosclerotic renal artery stenosis

Chapter 7 The effect of restoring renal patency on renal function in patients with hypertension and atherosclerotic renal artery stenosis. A systematic review.

Chapter 8 Main findings in perspective

Cgapter 9 Summary

Chapter 10 Samenvatting

Dankwoord

Curriculum vitae 



\section{Chapter 1}

Introduction 



\section{Introduction}

Hypertension is an important threat to public health affecting about $26 \%$ of the world's adult population, a number which is still rising. ${ }^{1}$ When left untreated, hypertension greatly enhances the risk of cardiovascular and renal disease. In developed countries, high blood pressure $(11 \%)$ is the second most common potentially modifiable risk to loss of healthy life, only the use of tobacco $(12 \%)$ ranking higher. ${ }^{2}$

In the great majority of patients with hypertension, no specific cause can be found. This "condition" is therefore called essential or primary hypertension. The pathogenesis of essential hypertension is multifactorial: genetic factors play an important role as do factors like obesity, smoking, insulin resistance, diet (sodium, potassium and calcium intake) and (excessive) use of alcohol. ${ }^{3,4}$ Comprehensive evaluation will identify a specific cause of the hypertension in approximately five percent of the patients with high blood pressure. The umbrella term for all specific causes is secondary hypertension. Renovascular hypertension is considered to be the second most common cause of secondary hypertension. ${ }^{5}$ About one percent of all individuals with elevated blood pressure in the general population has a stenosis in one or more of their renal arteries. $^{6-9}$ The presence of renal artery stenosis may lead to renovascular hypertension and renal dysfunction. ${ }^{10}$ Restoration of renal artery patency may, at least potentially, reverse hypertension and prevent (further) deterioration of kidney function. ${ }^{10}$

\section{The Maastricht angiography cohort}

Whereas the prevalence of renal artery stenosis is not so high in the general hypertensive population, this number rises dramatically in selected populations. For instance, in one study, $17 \%$ of the patients with severe or resistant hypertension that underwent diagnostic cardiac catheterisation had renal artery stenosis ${ }^{11}$ and the prevalence in patients entering dialysis programmes was estimated to be $11-14 \%$. ${ }^{12}$ The relatively high occurrence of renal artery stenosis in secondary care settings contrasts with the rather poor understanding of the interrelationships between renal artery lumen reduction, renal perfusion pressure and renal blood flow on the one hand and the eventual consequences of this, hypertension and renal dysfunction, on the other. This consideration has been the major impetus for the start of studies into renal blood flow in the University Hospital Maastricht. As of February 1994, all patients who are referred to our clinic for evaluation of their hypertension are subjected to a standard diagnostic protocol. This includes renal angiography with selective renal blood flow measurements and arterial and renal venous blood sampling when patients meet one or more of the following criteria: hypertension despite two or more antihypertensive drugs, accelerated hypertension, documented atherosclerotic vascular disease in two or more vascular beds, presence of an abdominal bruit or an unexplained impairment of renal function in response to antihypertensive treatment. In many of these patients endothelial function has been assessed by means of infusion of vasoactive substances. ${ }^{13-17}$ The percentage 
of patients that agree to participate is high: $98 \%$ patients who underwent selective baseline blood flow measurements consented to infusion of agents. Over time, the protocol has been adapted and extended in order to be able to elucidate other diagnostic and pathophysiological issues. From mid-1996 on, patients have been asked to donate a blood sample for genetic analysis. Since April 1999, measurements of intima-media thickness of the common carotid artery and aortic and brachial pulse wave velocity have been included in the protocol. Further, as part of the RADISH study, which investigated the value of computed tomographic angiography (CTA) and magnetic resonance angiography (MRA) for the diagnosis of renal artery stenosis, patients were asked to undergo CTA and MRA on the day prior to angiography between October 1998 and October 2001. ${ }^{18}$ In addition, renal scintigraphy and/or renal ultrasound were commonly applied as non-invasive screenings tests. Nowadays, these latter two tests have been largely abandoned in our hospital because of low sensitivity for the diagnosis of renal artery stenosis. ${ }^{19}$ Figure 1.1. details the diagnostic protocol of patients with hypertension referred to our outpatient clinic and Table 1.1 shows the characteristics of the angiography cohort.

Figure 1.1 Schematic outline of the diagnostic protocol of the Maastricht Angiography cohort.

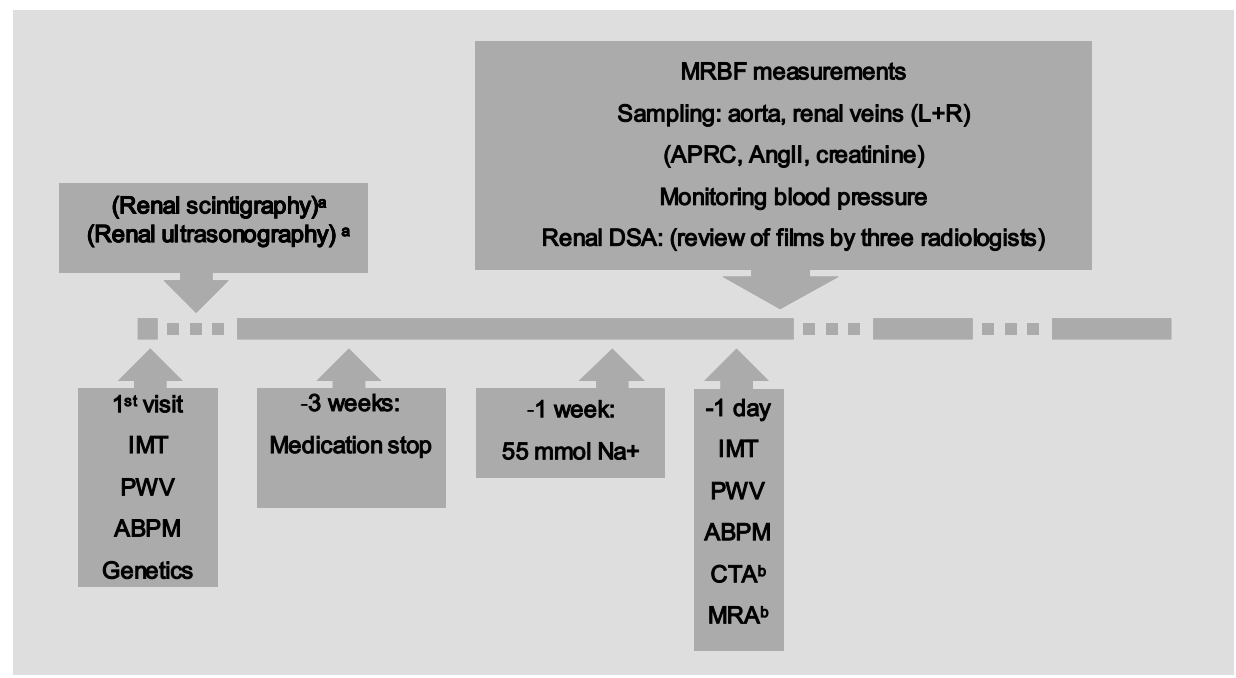

${ }^{a}$ whether this test was performed was to the discretion of the physician; ${ }^{b}$ these tests were performed only in patients who participated in the RADISH study. Dotted lines indicate that the duration of these time periods was not fixed. Usually the time between the first visit to the outpatient clinic and renal angiography did not exceed twelve months.

Table $1.1 \quad$ Patient characteristics.

\begin{tabular}{lccccc} 
& RAS- & FMD & ARAS & FMD and ARAS & Total \\
& $\mathrm{n}=303$ & $\mathrm{n}=29$ & $\mathrm{n}=154$ & $\mathrm{n}=4$ & $\mathrm{n}=490$ \\
\hline Age $\pm \mathrm{SD}$ & $50 \pm 13$ & $51 \pm 10$ & $61 \pm 10$ & 44 & $54 \pm 13$ \\
Gender $(\%$ male $)$ & 53 & 27 & 64 & 50 & 55
\end{tabular}




\section{Outline of this thesis}

The studies that form the main body of this thesis investigated diagnostic, pathophysiological and therapeutic aspects of renal artery stenosis in the Maastricht angiography cohort. These studies were designed and conducted to assess whether these aspects could 1) discern patients with renal artery stenosis out of a group of patients with moderate to severe hypertension in a secondary/tertiary care population and/or 2) shed light on the pathophysiology of renal artery stenosis and/or renovascular hypertension.

The gold standard for the diagnosis of renal artery stenosis still is intra-arterial digital subtraction angiography. However, this cannot be used routinely considering its invasive nature and costs. Unfortunately, none of the noninvasive screenings tests has proven to satisfy the requirements yet. ${ }^{18}$ Hence, patients are selected for renal angiography on the basis of clinical clues obtained in anamnesis and physical examination. Most textbooks and review articles that have proposed diagnostic strategies refer to either the clinical clues formulated in the 1987 report of the Working Group on Renovascular Hypertension or the Mann-Pickering criteria. ${ }^{9,20}$ Yet, the diagnostic accuracy of these clues has not been evaluated properly. Chapter 2 describes the prevalence and predictive value of these clues in our cohort. In addition, we tested whether a model incorporating clinical clues and risk factors for cardiovascular disease in general, would be able to select patients in whom digital subtraction angiography should be performed. In Chapter 3, the question has been addressed whether the prediction of the presence of renal artery stenosis can be further improved by knowledge about structural (intima media thickness of the common carotid artery) and functional (aortic pulse wave velocity) vessel wall properties and more accurate blood pressure measurements (24 hour ambulatory blood pressure measurements).

It is still largely unknown if and to what extent genetic abnormalities contribute to the development of atherosclerotic renal artery disease. Polymorphisms in genes of the renin-angiotensin system and endothelial nitric oxide synthase rank high among the potential candidates and their association with atherosclerotic renal artery stenosis is reported in Chapter 4. Because association studies are especially prone to (selection) bias, the prevalence of the polymorphisms was also studied in an independent sample of patients from a general care practice.

Evaluation of split renal function in patients with elevated blood pressure may be useful as a screening procedure to detect (unilateral) renal artery stenosis. The implicit assumption underlying diagnostic tests making use of this principle, is that, in the absence of a hemodynamically significant renal artery stenosis, blood flow through the kidneys will be roughly symmetrical. Whether this is truly the case has been investigated in Chapter 5 . Chapter 6 discusses differences between kidneys supplied by (unilaterally 
stenosed) atherosclerotic renal arteries and kidneys contralateral to these kidneys with respect to renal blood flow and its functional characteristics.

In Chapter 7 an excursion is made to the therapy of renal artery stenosis. The goals of restoration of renal artery patency, are nowadays usually two-fold: to improve blood pressure control and to stabilize renal function. The interest in the possibe effects on renal function is however of relatively recent date. None of the published randomized clinical trials included an adequate number of patients with sufficient long duration of follow-up to reach a definite conclusion about the effect on renal outcome on its own. Chapter 7 presents a meta-analysis of all available evidence of the effect of revascularisation on intermediate renal outcome.

A general discussion and summary of the main findings of this thesis are provided in Chapter 8 and 9, Chapter 10 provides a summary in Dutch. 


\section{References}

1. Kearney PM, Whelton M, Reynolds K, Muntner P, Whelton PK, He J. Global burden of hypertension: analysis of worldwide data. Lancet. 2005;365:217-23.

2. Ezzati M, Lopez AD, Rodgers A, Vander Hoorn S, Murray CJ. Selected major risk factors and global and regional burden of disease. Lancet. 2002;360:1347-60.

3. Staessen JA, Wang J, Bianchi G, Birkenhager WH. Essential hypertension. The Lancet. 2003;361:1629-41.

4. Carretero OA, Oparil S. Essential hypertension. Part I: definition and etiology. Circulation. 2000;101:329-35.

5. 2003 European Society of Hypertension-European Society of Cardiology guidelines for the management of arterial hypertension. J Hypertens. 2003;21:1011-53.

6. Berglund G, Andersson O, Wilhelmsen L. Prevalence of primary and secondary hypertension: studies in a random population sample. Br Med J. 1976;2:554-6.

7. Sigurdsson JA, Bengtsson C, Tibblin E, Wojciechowski J. Prevalence of secondary hypertension in a population sample of Swedish women. Eur Heart J. 1983;4:424-33.

8. Lewin A, Blaufox MD, Castle H, Entwisle G, Langford H. Apparent prevalence of curable hypertension in the Hypertension Detection and Follow-up Program. Arch Intern Med. 1985; 145:424-7.

9. Detection, evaluation, and treatment of renovascular hypertension. Final report. Working Group on Renovascular Hypertension. Arch Intern Med. 1987;147:820-9.

10. Safian RD, Textor SC. Renal-artery stenosis. N Eng/ J Med. 2001;344:431-42.

11. Buller CE, Nogareda JG, Ramanathan K, Ricci DR, Djurdjev O, Tinckam KJ, Penn IM, Fox RS, Stevens LA, Duncan JA, Levin A. The profile of cardiac patients with renal artery stenosis. J Am Coll Cardiol. 2004;43:1606-13.

12. Preston RA, Epstein M. Ischemic renal disease: an emerging cause of chronic renal failure and end-stage renal disease. J Hypertens. 1997;15:1365-77.

13. Wierema TK, Houben AJ, de Leeuw PW. Acetylcholine-induced vasodilatation in the human hypertensive kidney: inhibition by muscarinic receptor antagonism. $J$ Hypertens. 1997;15:1649-51.

14. Wierema TK, Postma CT, Houben AJ, Kroon AA, Thien T, Smits P, de Leeuw PW. Adenosine-induced renal vasodilatation is prolonged in renal artery stenosis. $J$ Hypertens. 1998;16:2109-12.

15. Wierema TK, Houben AJ, Kroon AA, Koster D, van der Zander K, van Engelshoven JM, de Leeuw PW. Nitric oxide dependence of renal blood flow in patients with renal artery stenosis. J Am Soc Nephrol. 2001;12:1836-43.

16. van Uum SH, Houben AJ, Hermus AR, Kroon AA, Walker BR, Sweep CG, Smits P, de Leeuw $\mathrm{PW}$, Lenders JW. Acute intrarenal administration of cortisol has no effect on renal blood flow in hypertensive individuals. J Hypertens. 2002;20:2275-83.

17. van der Zander K, Houben AJHM, Kroon AA, Wierema TKA, Fuss-Lejeune MJMJ, Koster D, de Leeuw PW. Does Brain Natriuretic Peptide Have a Direct Renal Effect in Human Hypertensives? Hypertension. 2003;41:119-23.

18. Vasbinder GB, Nelemans PJ, Kessels AG, Kroon AA, Maki JH, Leiner T, Beek FJ, Korst MB, Flobbe K, de Haan MW, van Zwam WH, Postma CT, Hunink MG, de Leeuw PW, van Engelshoven JM. Accuracy of computed tomographic angiography and magnetic resonance angiography for diagnosing renal artery stenosis. Ann Intern Med. 2004;141:674-82.

19. Vasbinder GBC, Nelemans PJ, Kessels AG, Kroon AA, De Leeuw PW, Van Engelshoven JM. Diagnostic tests for renal artery stenosis in patients suspected of having renovascular hypertension: a meta-analysis. Ann Intern Med. 2001;135:401-11.

20. Mann SJ, Pickering TG. Detection of renovascular hypertension. State of the art: 1992. Ann Intern Med. 1992;117:845-53. 



\section{Chapter 2}

The predictive value of established clinical clues for the diagnosis of renal artery stenosis in clinical practice

Marieke van Onna, Patty J. Nelemans, Abraham A. Kroon, Alfons G.H. Kessels, Karin Flobbe, Michiel W. de Haan, Peter W. de Leeuw 


\section{Abstract}

The gold standard for the diagnosis of renal artery stenosis is digital subtraction angiography. However, this cannot be used routinely considering its invasive nature and costs. Presently, patients are selected for renal angiography on the basis of clinical clues. Yet, the diagnostic accuracy of these clues has not been evaluated properly. Aims of this study were: 1 . to evaluate the ability of established clinical clues as proposed by the 1987 report of the Working Group on Renovascular Hypertension and the Mann-Pickering criteria to predict the presence of renal artery stenosis 2. to develop a model for more efficient patient selection for renal angiography. Clinical clues and patient characteristics were abstracted from the medical records of all patients who were referred to our outpatient clinic for the evaluation of hypertension and who had undergone renal angiography $(1998-2003, n=326)$. Renal angiography films were examined by three radiologists. Most clinical clues had only limited predictive value because of a low prevalence, lack of discriminative ability or lack of additional information over and above other patient characteristics. Three clues (length difference between kidneys $\geq 10 \%$, the presence (and extent of) extra-renal atherosclerosis and recent onset of hypertension), proved to be independent predictors. A model combing these three clues with information regarding age, pulse pressure and smoking achieved an optimism-corrected area under the receiver-operating characteristic curve of 0.75 . Our model may be used as a guide to select patients for angiography and a reduction of the number of patients undergoing angiography may be feasible. 


\section{Introduction}

The prevalence of the renal artery stenosis among unselected patients with hypertension is low. Yet, clinicians often search thoroughly for this condition, since it is one of the few causes of (potentially) reversible hypertension and/or renal failure. The gold standard for diagnosis is digital subtraction angiography, but in view of its costs, invasive nature and potentially serious complications, angiography cannot be used routinely. Major effort has been put in the development and evaluation of non-invasive screening tests during the last decades. Unfortunately, however, none of these tests has an adequate diagnostic accuracy. ${ }^{1,2}$ Hence, we must still rely on clinical features in the process of selecting candidates for angiography. Most textbooks and review articles that have proposed diagnostic strategies refer to either the clinical clues formulated in the 1987 report of the Working Group on Renovascular Hypertension ${ }^{3}$ or the MannPickering criteria, ${ }^{4}$ which are primarily based on one systematic survey. ${ }^{5}$ Remarkably though, the diagnostic accuracy of these criteria has not been properly evaluated. Therefore, the present study assessed the predictive value of these clinical clues in patients clinically suspected of having renovascular hypertension. In addition we tested whether a model incorporating clinical clues and risk factors for cardiovascular disease in general, would be able to select patients in whom digital subtraction angiography should be performed.

\section{Materials and methods}

\section{Patients}

The study group consisted of consecutive patients who had been referred to the outpatient clinic of the department of internal medicine of the University Hospital Maastricht for the evaluation of hypertension and who had undergone digital subtraction renal angiography between October 1998 and October 2003. The outpatient clinic of the department of internal medicine is a referral center for hypertension, but since the University Hospital Maastricht is the only hospital in the Maastricht region, general practitioners also refer patients to our clinic. Patients were eligible for this study if they were between 18 and 75 years of age, had a diastolic blood pressure equal to or exceeding $95 \mathrm{mmHg}$, and had one or more clinical characteristics suggestive of the presence of renovascular hypertension ${ }^{3,4}$ and/or signs commonly associated with renal artery disease like high serum cholesterol concentration, low serum potassium concentration, and/or left ventricular hypertrophy. Patients suspected of restenosis after treated renovascular hypertension were also included (Table 2.1). It should be noted that the criterion "hypertension refractory to three-drug treatment" as defined by the Working Group on Renovascular Hypertension was changed into "hypertension refractory to twodrug treatment", as was proposed by the DRASTIC investigators. ${ }^{6}$ Finally, suspicion of renovascular hypertension had to have been the main indication for angiography. Subjects with previously diagnosed yet untreated renal artery stenosis were excluded. 


\section{Data Collection}

Data were collected using standardized forms, which included information on the abovementioned clinical clues as well as age, gender, body height, weight, smoking habits (ever-smoker versus never-smoker), duration of hypertension (in years), number of antihypertensive drugs used by the patient, systolic and diastolic blood pressure (defined as the average of the office blood pressure measurements obtained during the visit in which the decision was taken to perform angiography), concentrations of creatinine $(\mu \mathrm{mol} / \mathrm{l})$, cholesterol $(\mathrm{mmol} / \mathrm{l})$ and potassium $(\mathrm{mmol} / \mathrm{l})$ and results of renal ultrasound (length of both kidneys) and echocardiography. Diabetes mellitus was considered to be present when the patient used insulin or oral anti-diabetic drugs, or when postprandial plasma glucose levels exceeded $11.0 \mathrm{mmol} / \mathrm{l}$. The diagnosis of hyperlipidemia was based on a cholesterol level above $7.0 \mathrm{mmol} / \mathrm{l}$ or the use of cholesterol-lowering drugs.

\section{Evaluation of Digital Subtraction Angiography}

Two experienced radiologists evaluated all angiographic films independently. They used standardized forms to report on whether or not a lesion was present as well as on the site, nature of the abnormality and, only in case of atherosclerotic disease, the percentage of (maximum) luminal reduction. If the two estimates of the degree of stenosis differed by $10 \%$ or more (of the highest assessment), the opinion of a third radiologist was decisive. A patient was considered to have renal artery stenosis if the angiogram showed fibromuscular dysplasia (FMD) or $\geq 50 \%$ luminal reduction in case of atherosclerotic disease, consistent with our hospitals policy to perform revascularisation in these cases.

\section{Statistical Analysis}

\section{Predictive ability of clinical clues and derivation of the prediction model}

We explored the prevalence and predictive abilities of individual clinical clues by univariable regression analysis. Next, multivariable analysis was performed in order to calculate the contribution of each factor while accounting for all other factors in the model. We entered all clinical clues and risk factors into the model except for: 1 clinical clues that were present in less than $5 \%$ of the patients; 2 candidate predictors that (partially) measure the same concepts as other candidate predictors, these predictors were combined; and 3 candidate predictors that showed high collinearity with other candidate predictors. In these cases of combinations of highly correlated variables, we tested which variable performed best, as determined by the change in -2 Log Likelihood following the addition of such a variable to a model containing all other candidate predictors. Furthermore we introduced interaction terms into the model to test whether interactions between blood pressure and age and between blood pressure and smoking were present. We checked for any nonlinear relationship between presence of renal artery stenosis and any continuous variable. The final model was obtained by 
multivariable logistic regression analysis using a backward elimination procedure variables with the $p$-value for removal set at 0.1 .

\section{Model evaluation}

The reliability of predictions by the model was tested by the Hosmer-Lemeshow goodness-of-fit test. The predictive ability of the model was assessed by the area under the receiver operating characteristic curve (AUC). The internal validity of the model was evaluated by the use of bootstrapping. For this, the multivariable logistic regression analysis with backward elimination of variables was replicated in 200 bootstrap samples. The AUC of each of these 200 models was compared to the AUC of the model in the original dataset. The mean absolute difference between these 200 values and the value resulting from the original dataset is an estimation of the optimism in the fit of the model and the AUC was adjusted for this estimation. ${ }^{7}$ We estimated the degree of overfitting likely to be present by the method by Van Houwelingen and Le Cessie ${ }^{8}$ and adjusted the regression coefficients accordingly. The rationale behind this adjustment is to improve the calibration of the model, i.e. to prevent low predictions from being too low and high predictions from being too high. ${ }^{7}$ To enable the use of the model in practice, the predictor variables were given weighted scores based on their regression coefficients. We determined the predicted probability of renal artery stenosis for all possible sum scores. $^{9}$

\section{Missing data}

Missing values of candidate predictors were handled by multiple imputation from an imputation model that contained the dependent variable (presence or absence of renal artery stenosis), all candidate variables that were intended to enter into the prediction model and factors that were related to the occurrence of missing data. ${ }^{10}$ For any missing value, ten values were drawn from the posterior distribution of the imputation model. ${ }^{11}$ Thus, ten completed data sets were created. All multivariable analyses were conducted on each of the ten datasets and the results and standard errors were combined. ${ }^{12,13}$ Analyses were performed with SPSS 11.5 , StataSE 8.0 and Amelia. ${ }^{14}$

This study was approved by the Institutional Review Board of the Maastricht University Hospital.

\section{Results}

Between October 1998 and October 2003, 372 patients had undergone renal angiography, of whom 326 were judged eligible for this study. Main reasons for exclusion were: age lower than 18 or higher than 75 years, absence of clinical clues suggesting renovascular hypertension and a history of untreated renal artery abnormalities. Angiography revealed atherosclerotic renal artery stenosis of $50 \%$ or 
more in 49 patients, FMD in fourteen patients and a combination of atherosclerotic and fibromuscular abnormalities in one patient.

The prevalence of the clinical clues in the study population is presented in Table 2.1. The prevalence of most clues was rather low, i.e. below $25 \%$. Criteria which were more frequently observed were "hypertension refractory to appropriate treatment", "sudden onset or worsening of hypertension", "unexplained impairment of renal function", "raised serum cholesterol" and "left ventricular hypertrophy". Patients with renal artery stenosis presented with more inclusion criteria and also a higher number of clues according to the Working Group on Renovascular Hypertension than patients with patent arteries (mean \pm SD: $4 \pm 2$ vs. $3 \pm 2, p<0.0001$ and $2 \pm 1$ vs. $1 \pm 1, p<0.0001$, respectively).

The results of univariable analysis of all candidate predictors are presented in Table 2.2. Statistitical significant predictors $(p<0.05)$ of renal artery stenosis were: age, body mass index, pulse pressure, smoking, serum creatinine, and the criteria "unexplained renal impairment or renal disease", "kidney lenght difference", "presence of a renal bruit", "recent-onset hypertension" and "extra-renal atherosclerosis". The great majority of variables with $p>0.05$ had an odds ratio near 1 , indicating, in case of a dichotomous variable, that these risk factors appear with the same frequency in patients with and without renal artery stenosis.

Table 2.2 also shows the results of the multivariable analysis. The model obtained with backward elimination (simplified model) contained only six predictors of renal artery stenosis: age, pulse pressure, smoking, "kidney length difference", "recent onset hypertension" and "extra-renal artherosclerosis". The AUC of the full model was 0.81 $(95 \%-\mathrm{Cl} 0.76-0.87)$, that of the simplified model $0.79(95 \%-\mathrm{Cl} 0.73-0.85)$. The small difference between these values suggested that the variables that were deleted during stepwise variable selection did not contribute much to the predictive ability. The bootstrap adjusted AUC of the simplified model was 0.75 .

The model fitted well according to the Hosmer-Lemeshow goodness-of-fit test $(p=0.57)$. Figure 2.1 presents the agreement between the predicted probability of renal artery stenosis by the simplified model versus the observed probability of renal artery stenosis. The overall calibration was quite good, except in the highest categories (with predicted probabilities $\geq 70 \%, 2 \%$ of the study group), where predicted probabilities were lower than observed probabilities. The AUC for patients with atherosclerotic disease was higher than for patients with FMD $(0.83(95 \%-\mathrm{Cl} 0.76-0.89)$ versus $0.68(95 \%-\mathrm{Cl}$ 0.59-0.78)). To obtain the predictor "kidney length difference", the patients will have to undergo additional testing. In a post-hoc analysis, we determined the AUC of the model without this predictor. The AUC was $0.77(95 \%-\mathrm{Cl} 0.71-0.89)$ and 0.74 after bootstrap correction. (Figure 2.1). 
Table 2.1 Inclusion criteria

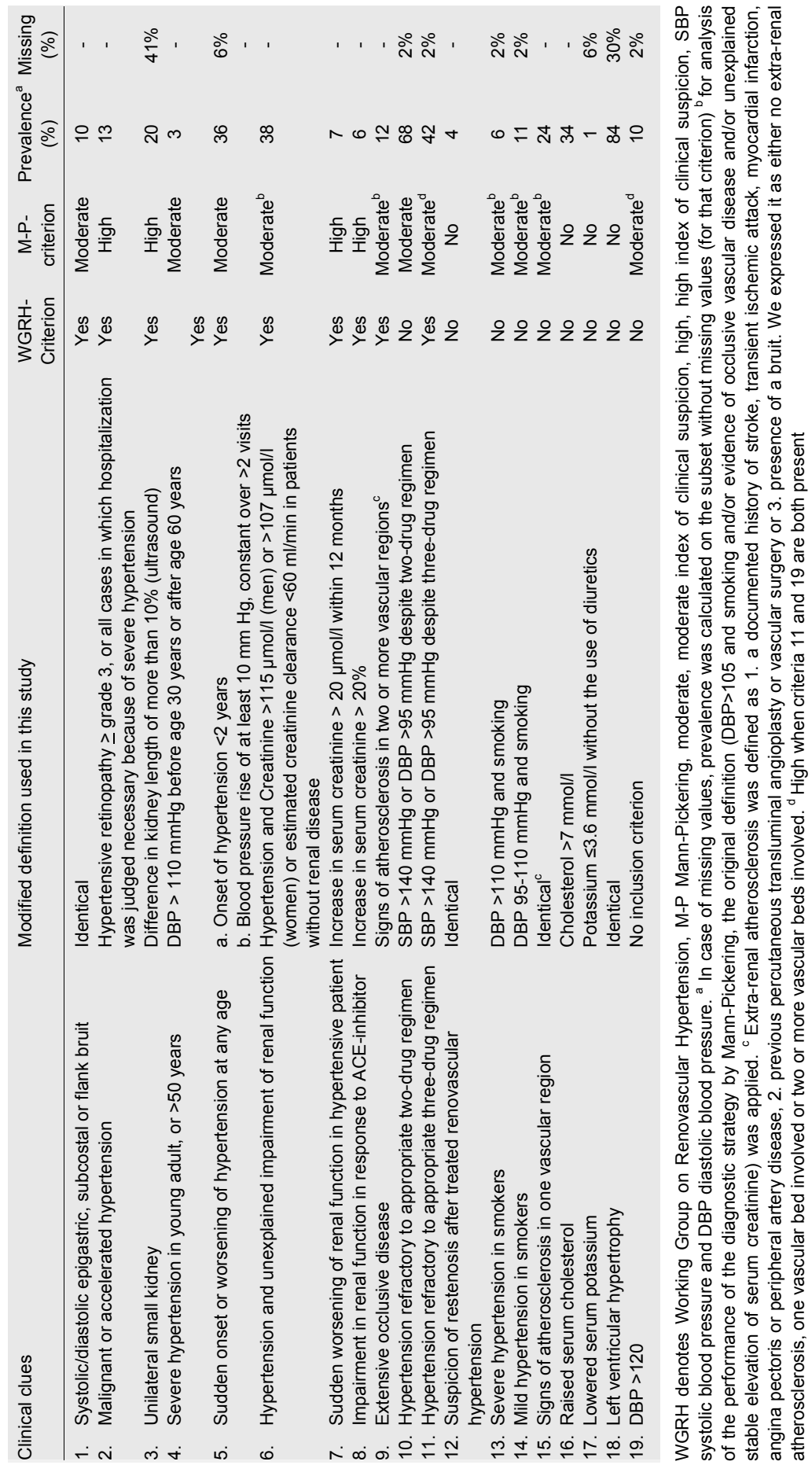


$24 \mid$ Chapter 2

Table 2.2 Predictive ability of clinical clues.

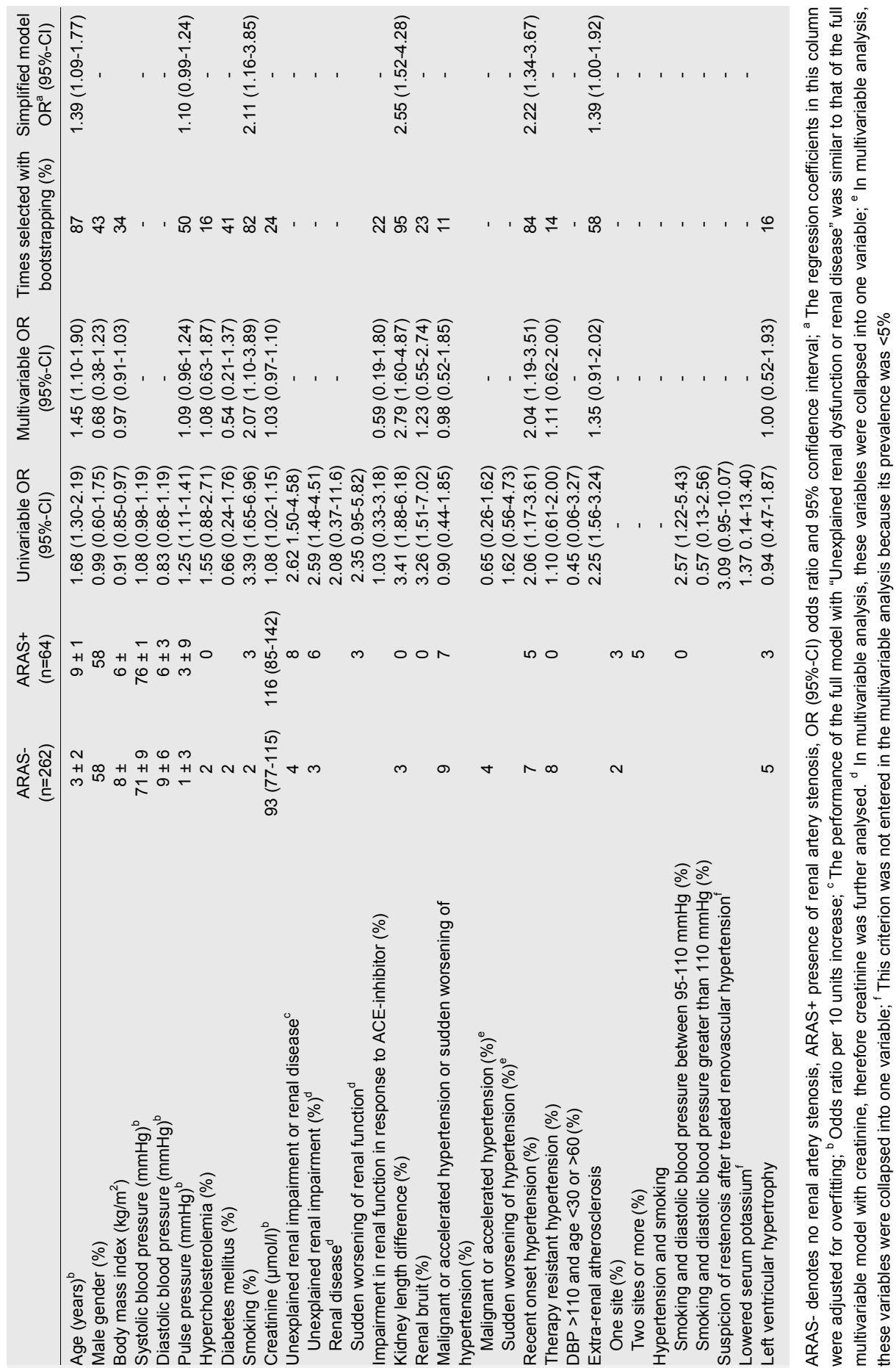


Figure 2.1 The agreement between the predicted and the observed probabilities of renal artery stenosis in patients suspected of renovascular hypertension.

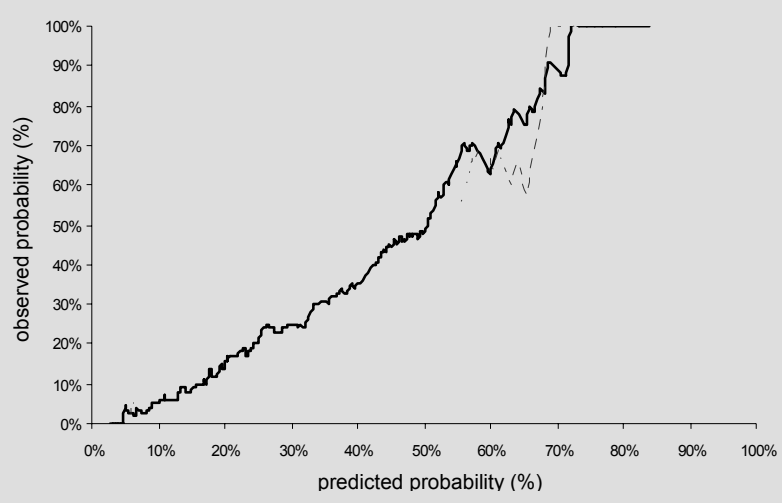

Agreement between the predicted probabilities of renal artery stenosis (the horizontal axis) and observed probabilities of renal artery stenosis (on the vertical axis). The thick line represents the smoothed calibration curve of predictions when information regarding renal lengths is available, the dotted line when this information is not available.

To obtain the predicted probability for renal artery stenosis in a patient, the first step is to add the scores that are associated with the level of the predictors (Table 2.3).

Table 2.3 Sum score.

\begin{tabular}{lcc} 
Predictor & & Number of points \\
\hline Age & $<30$ & -2 \\
& $30-39$ & 0 \\
& $40-49$ & 2 \\
& $50-59$ & 4 \\
Pulse pressure & $60-69$ & 6 \\
& $70-75$ & 8 \\
& $<50$ & -1 \\
& $50-59$ & 0 \\
& $60-79$ & 1 \\
Current or former smoker? & $80-99$ & 2 \\
& $100-109$ & 3 \\
Recent onset of hypertension & $\geq 110$ & 4 \\
& no & 0 \\
Number of vascular beds with manifest & yes & 5 \\
atherosclerosis (excluding renal vasculature) & no & 0 \\
>10\% difference between length of the kidneys as & yes & 5 \\
determined by renal ultrasound ? & 0 & 0 \\
& 1 & 2 \\
& $\geq 2$ & 4 \\
& absent & 0 \\
& present & 6 \\
& unknown & 0
\end{tabular}


This sum score corresponds to a predicted probability as is presented in the Figure 2.2. The probability plot can be used when renal ultrasound is part of routine work-up and when it is not. One may decide to perform angiography only when the probability of renal artery stenosis is higher than a certain threshold value. Table 2.4 represents sensitivity, specificity, the number patients of that will be referred for angiography and the number of cases with renal artery stenosis that are missed for various cut-off points.

Figure 2.2 Predicted probability of renal artery stenosis in patients suspected of renovascular hypertension.

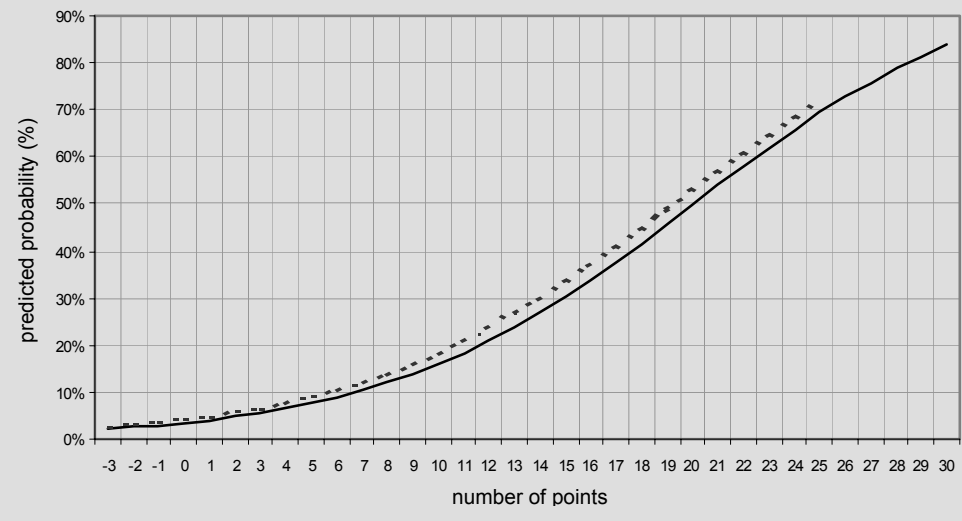

In order to obtain the risk estimate of a patient on renal artery the relevant numbers in the right column of Table 2.3 should be added. For each sum of scores, the probability of renal artery stenosis, as derived by logistic regression analysis (simplified model, Table 2.2), is plotted against the probability of renal artery stenosis. When a patient did undergo renal ultrasound, the bold curve applies, if not, the dotted one should be used. The probability can also by calculated with the following formula:

$$
\frac{\mathrm{e}^{\mathrm{S}}}{\mathrm{e}^{\mathrm{S}+1}}
$$

In case ultrasound performed was: $\mathrm{S}=-4.99+0.033^{*}$ age $+0.010^{*}$ pulse pressure + $0.75^{*}$ smoking behaviour $+0.80^{*}$ recent onset of hypertension $+0.33^{*}$ extra-renal atherosclerosis $+0.94^{*}$ kidney length difference $>10 \%$

In case ultrasound was not performed: $S=-4.738+0.032^{*}$ age $+0.010^{*}$ pulse pressure $+0.84^{*}$ smoking behaviour $+0.77^{\star}$ recent onset of hypertension + $0.33^{*}$ extra-renal atherosclerosis 
Table 2.4 The consequences of several threshold values for the predicted probability at which angiography is preformed.

\begin{tabular}{lrrcc}
$\begin{array}{l}\text { Predicted } \\
\text { probability (\%) }\end{array}$ & \multicolumn{2}{c}{ Sensitivity (\%) Specificity (\%) } & $\begin{array}{c}\text { Reduction of } \\
\text { angiographies (\%) }\end{array}$ & $\begin{array}{c}\text { Missed cases of renal } \\
\text { artery stenosis (\%) }\end{array}$ \\
\hline$>0$ & 100 & 0 & 0 & 0 \\
$>10$ & 93 & 38 & 38 & 11 \\
$>20$ & 74 & 69 & 60 & 29 \\
$>30$ & 49 & 85 & 74 & 43 \\
$>40$ & 29 & 91 & 86 & 59 \\
$>50$ & 20 & 97 & 89 & 69 \\
$>60$ & 10 & 99 & 95 & 81 \\
$>70$ & 5 & 100 & 98 & 90
\end{tabular}

\section{Discussion}

We evaluated the ability of several established and widely quoted clinical clues to predict the presence of renal artery stenosis in a population of consecutive patients suspected of having renovascular hypertension. Although the number of positive criteria was higher in patients with renal artery stenosis than in those without, most of these clues had only limited predictive value. Reasons for this finding were a low prevalence of these clues, lack of ability to discriminate between patients with and without renal artery stenosis, or lack of additional information over and above other patient characteristics. For example, several previous studies identified an abdominal bruit as an important predictor, ${ }^{5,16-19}$ a finding which we could confirm in univariable analysis. However, when all potential predictors were tested simultaneously using multivariable analysis, the odds ratio dropped from 3.26 to 1.23 , and was no longer statistically significant. Only three clues, namely a length difference between the kidneys exceeding $10 \%$, as diagnosed by renal ultrasound, the presence (and extent of) extra-renal atherosclerosis and onset of hypertension within two years, proved to be independent predictors of renal artery stenosis. A model that combined these three clues with information regarding age, pulse pressure and smoking had a reasonable predictive value. The criterion "kidney length difference" involves testing that may not be part of the diagnostic work-up of renovascular hypertension in all centers. When this information is not available, the predictive ability of the model is lower but still fairly good.

The difficulty of the diagnostic work-up of patients in whom renovascular hypertension is suspected, is well reflected by the amount of articles proposing diagnostic strategies. The most popular ones is probably the Mann-Pickering strategy. Applying the classification by Mann and Pickering to our study group, led to 172 patients (among whom 45 with renal artery stenosis) with a high index of clinical suspicion, 118 with a moderate index (among whom twelve with renal artery stenosis) and 36 (among whom seven with renal artery stenosis) with a low index of suspicion. Mann and Pickering state that patients with a high index of clinical suspicion should undergo angiography, 
patients with a moderate index of clinical suspicion should undergo non-invasive screening, and patients with a low index should not be tested any further. We assumed a sensitivity of $69 \%$ and a specificity of $91 \%$ for the non-invasive screening test, as was found for the best non-invasive screening test in a recent comparative study between computed tomography angiography and magnetic resonance angiography. ${ }^{1}$ In that case, digital subtraction angiography would have been performed in 190 patients, and would have detected 53 out of the 64 cases with renal artery stenosis. This diagnostic strategy would have a sensitivity of $83 \%$ and a specificity of $48 \%$ in our study population, while reducing the number of angiographies from 326 to 190 , a reduction by $42 \%$. Using the prediction model as developed in the present study, a sensitivity of $83 \%$ is associated with a specificity of $59 \%$. In other words, to detect the same number of stenoses as with the Mann and Pickering strategy, 160 instead of 326 angiographies would have to be performed, a reduction by $51 \%$. The major disadvantage of the Mann and Pickering strategy is the incorporation of a screening test, with associated costs and relatively low gain in the absence of satisfactory noninvasive tests. If a better screening test would become available, the specificity of the strategy on its whole would rise.

Another well-known model is the prediction rule developed in the DRASTIC-study. ${ }^{18}$ The use of this prediction rule is restricted to patients with either treatment resistant hypertension or an increase in serum creatinine during therapy with an ACE-inhibitor. In this respect, we believe that the prediction rule developed in this study better approaches common clinical practice since it is applicable to all patients who have hypertension and at least one clinical clue pointing to renal artery stenosis. For example, it is likely that a clinician will suspect renovascular hypertension in a smoking 65-year old male patient with a renal bruit even when his blood pressure is controlled with two drugs. Moreover, our study enabled evaluation of the use of potential predictors that were not investigated in the model of the DRASTIC-investigators. An overview of differences between our model and the DRASTIC rule as well as the Mann Pickering strategy is presented in Table 2.5 .

There are more studies on the predictive value of clinical clues for the presence of renal artery stenosis, but the implementation of findings from these studies is hampered by the use of screening tests with sub-optimal characteristics, ${ }^{20-23}$ the development on small data-sets ${ }^{17,24}$ or lack of proof of renal artery stenosis by digital subtraction angiography. ${ }^{24,25}$

The present study also has limitations. First of all, a relatively high number of patients did not undergo renal ultrasound (41\%) and/or echocardiography (30\%). Renal duplex ultrasound was abandoned as a screeningtest during the study period because of its low sensitivity for the diagnosis of renal arty stenosis and the amount of missing data was therefore expected. However, we dealt with these missing values using a statistical technique that gives unbiased estimates of the odds ratio's assuming that the probability of missing data on a certain variable is unrelated to the value of that certain variable 
after controlling for observed variables, in other words when data are missing at random (MAR). ${ }^{12}$ Secondly, the number of candidate predictors is relatively high in comparison to the number of patients with renal artery stenosis. We partly tackled this problem by reducing the number of candidate predictors by combining criteria that (partially) measure the same concepts and by adjusting the predictions by the model with shrinkage. Whether these measures were sufficient can only be assessed by external validation, but the stability of the variable selection across the bootstrap samples was encouraging. Since it is well known that any prediction model performs best in the data set it was built on, external validation is always needed. Thirdly, we acknowledge that preferably any test in this field should aim at detecting only those patients with renal artery stenosis who, at least potentially, benefit from treatment. However, adopting (partly) reversible hypertension as outcome in a prediction model would neglect the fact that revascularisation sometimes fails because of technical reasons, restenosis and the fact that patients may be diagnosed with renal artery stenosis at a time when secondary changes have already diminished the chance of a favorable outcome. Finally, all predictors, with the exception of "recent onset of hypertension", seem to be related more to atherosclerosis than to renovascular hypertension per se, probably because atherosclerotic abnormalities were much more prevalent than FMD. Not surprisingly, therefore, the AUC for patients with atherosclerotic disease was higher than for patients with FMD, indicating that our model performed better at selecting patients with atherosclerotic than with fibromuscular abnormalities. It was not feasible, however, to make a distinctive model for the prediction of FMD because of its low prevalence.

Our model may be used as a guide to select patients for angiography. In order to do so, a certain cut-off value for the predicted probability above which patients are subjected to angiography should be decided on. The choice of such a threshold value is always a trade-off between sensitivity and specificity of a test. Cutting back on specificity will unduly subject more patients without renal artery stenosis to digital subtraction angiography, while compromising on sensitivity will increase the number of patients with renal artery stenosis that are declared healthy. In order to make a good choice, the consequences of either strategy should be taken into account. Subjecting more patients to angiography is costly and leads in a minority of patients to serious complications like deterioration of renal function by contrast agents or cholesterol embolism. On the other hand, when the diagnosis of renal artery stenosis is missed, patients do not benefit from specific treatment which is in most cases revascularisation. However, whereas in terms of blood pressure, the value of revascularisation is well established in FMD, the merit for patients with atherosclerotic disease is much less clear. Until large prospective trials determine the benefits of revascularisation, it may be justified to give preference to decreasing the number of patients subjected to angiography over identifying all patients with renal artery stenosis. 
Table 2.5 Comparison between the present prediction model and other prediction models.

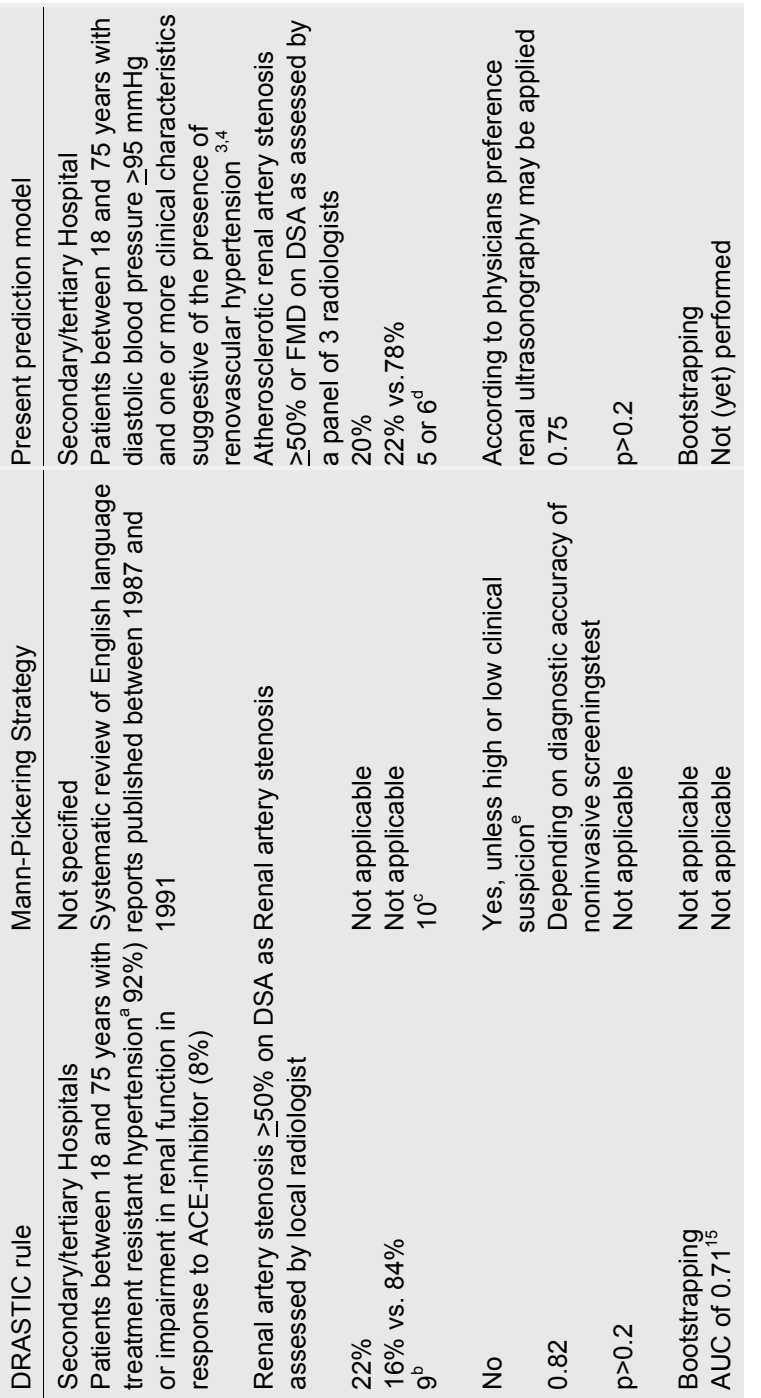

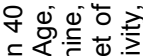

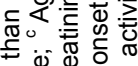
ষ্口. 要 ब

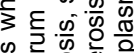
为

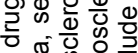
$\sim$ ․․․

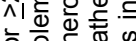
政 का 为

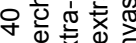
प产市 ब) ब

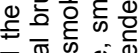

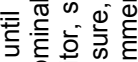
온융을 엉 시은

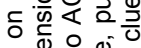

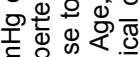
ह 오음응

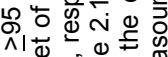

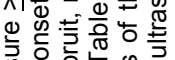

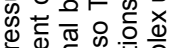

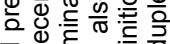
으의 응 $\frac{\omega}{5} \mathbb{D} \Phi$ 은 응 造 $\frac{\bar{N}}{\omega} \bar{c}$ क्ष

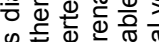
뉸 ब元政心 过 क ธิ㇒

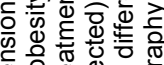

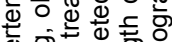
¿ำ

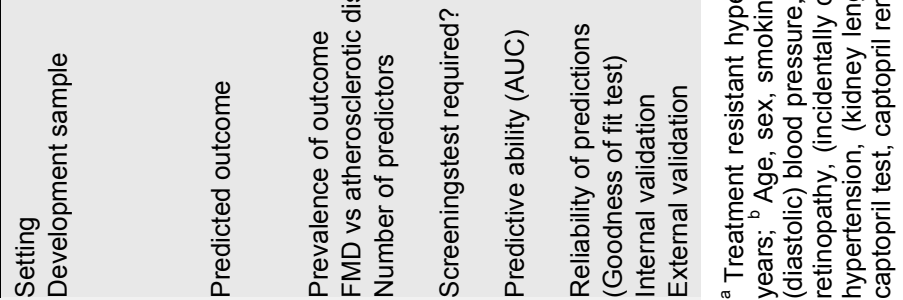




\section{References}

1. Vasbinder GB, Nelemans PJ, Kessels AG, Kroon AA, Maki JH, Leiner T, Beek FJ, Korst MB, Flobbe K, de Haan MW, van Zwam WH, Postma CT, Hunink MG, de Leeuw PW, van Engelshoven JM. Accuracy of computed tomographic angiography and magnetic resonance angiography for diagnosing renal artery stenosis. Ann Intern Med. 2004;141:674-82.

2. Vasbinder GBC, Nelemans PJ, Kessels AG, Kroon AA, De Leeuw PW, Van Engelshoven JM. Diagnostic tests for renal artery stenosis in patients suspected of having renovascular hypertension: a meta-analysis. Ann Intern Med. 2001;135:401-11.

3. Detection, evaluation, and treatment of renovascular hypertension. Final report. Working Group on Renovascular Hypertension. Arch Intern Med. 1987;147:820-9.

4. Mann SJ, Pickering TG. Detection of renovascular hypertension. State of the art: 1992. Ann Intern Med. 1992;117:845-53.

5. Simon N, Franklin SS, Bleifer $\mathrm{KH}$, Maxwell $\mathrm{MH}$. Clinical characteristics of renovascular hypertension. JAMA. 1972;220:1209-18.

6. Van Jaarsveld BC, Krijnen P, Derkx FH, Deinum J, Woittiez AJ, Postma CT, Schalekamp MA. Resistance to antihypertensive medication as predictor of renal artery stenosis: comparison of two drug regimens. J Hum Hypertens. 2001;15:669-76.

7. Harrell FE, Jr., Lee KL, Mark DB. Multivariable prognostic models: issues in developing models, evaluating assumptions and adequacy, and measuring and reducing errors. Stat Med. 1996;15:361-87.

8. Van Houwelingen JC, Le Cessie S. Predictive value of statistical models. Stat Med. 1990;9:1303-25.

9. Sullivan LM, Massaro JM, D'Agostino RB, Sr. Presentation of multivariate data for clinical use: The Framingham Study risk score functions. Stat Med. 2004;23:1631-60.

10. Van Buuren S, Boshuizen HC, Knook DL. Multiple imputation of missing blood pressure covariates in survival analysis. Stat Med. 1999;18:681-94.

11. King G, Honaker J, Joseph A, Scheve K. Analyzing incomplete political science data: an alternative algorithm for multiple imputation. American Political Science Review. 2001;95: 49-69.

12. Allison PD. Missing data. Thousand Oaks, CA: Sage Publications Inc.; 2001.

13. Schafer JL, Graham JW. Missing data: our view of the state of the art. Psychol Methods. 2002;7:147-77.

14. Honaker J, Joseph A, King G, Scheve K, Singh N. Amelia: a program for missing data (Windows version) Cambridge, MA: Harvard University ; 2001. Available as freeware from http://GKing.Harvard.edu. Accessed 10 Sept 2004.

15. Krijnen P, Steyerberg EW, Postma CT, Flobbe K, de Leeuw PW, Hunink MG. Validation of a prediction rule for renal artery stenosis. J Hypertens. 2005;23:1583-8.

16. Perera GA, Haelig AW. Clinical characteristics of hypertension associated with unilateral renal disease. Circulation. 1952;6:549-52.

17. Svetkey LP, Helms MJ, Dunnick NR, Klotman PE. Clinical characteristics useful in screening for renovascular disease. South Med J. 1990;83:743-7.

18. Krijnen P, Van Jaarsveld BC, Steyerberg EW, Man in 't Veld AJ, Schalekamp MA, Habbema JD. A clinical prediction rule for renal artery stenosis. Ann Intern Med. 1998;129:705-11.

19. Hunt JC, Sheps SG, Harrison EG, Jr., Strong CG, Bernatz PE. Renal and renovascular hypertension. A reasoned approach to diagnosis and management. Arch Intern Med. 1974; 133:988-99.

20. Vaughan ED, Jr., Case DB, Pickering TG, Sosa RE, Sos TA, Laragh JH. Clinical evaluation of renovascular hypertension and therapeutic decisions. Urol Clin North Am. 1984;11: 393-407.

21. Horvath JS, Waugh RC, Tiller DJ, Duggin GG. The detection of renovascular hypertension: a study of 490 patients by renal angiography. $Q J$ Med. 1982;51:139-46.

22. Bijlstra PJ, Postma CT, De Boo T, Thien T. Clinical and biochemical criteria in the detection of renal artery stenosis. J Hypertens. 1996;14:1033-40. 
23. Alcazar JM, Rodicio JL. How to handle renovascular hypertension. $J$ Hypertens. 2001;19:2109-11.

24. Shurrab AE, Mamtora H, O'Donoghue D, Waldek S, Kalra PA. Increasing the diagnostic yield of renal angiography for the diagnosis of atheromatous renovascular disease. $\mathrm{Br} J$ Radiol. 2001;74:213-8.

25. Anderson GH, Jr., Blakeman N, Streeten DH. Prediction of renovascular hypertension. Comparison of clinical diagnostic indices. Am J Hypertens. 1988;1:301-4. 


\section{Chapter 3}

The additional value of measuring IMT, PWV, and ambulatory blood pressure in the diagnosis of renal artery stenosis

Marieke van Onna, Alfons J. Houben, Abraham A. Kroon, Monique J. Fuss-Lejeune, Peter W. de Leeuw 


\section{Abstract}

The gold standard for the diagnosis of renal artery stenosis is digital subtraction angiography. However, this cannot be used routinely considering its invasive nature and costs. This study investigated wether intima media thickness (IMT), pulse wave velocity (PWV) and ambulatory blood pressure (ABPM) may contribute to the prediction of renal artery stenosis when added to previously identified predictors. In order to do so, IMT, PWV and ABPM were measured in 294 consecutive patients referred to the outpatient clinic of a tertiary referral hospital who underwent renal angiography because of clinical suspicion on renovascular hypertension. Multivariable regression analysis and the area under the receiver operating characteristic curve (AUC) were used to evaluate the added predictive value of IMT, PWV and ABPM. IMT, PWV and ABPM were associated with renal artery stenosis in univariable regression analysis. The AUC of a model with age, office pulse pressure, smoking, onset of hypertension within two years, extra-renal atherosclerosis and a difference in kidney length was 0.77 . When either IMT or PWV or ambulatory pulse pressure or any combination of the three was added, the predictive ability did not change. Our results do not support the use of IMT, PWV and ABPM for the assessment of risk on renal artery stenosis in an individual patient. 


\section{Introduction}

Common carotid intima media thickness (IMT) and aortic pulse wave velocity (PWV) are markers of structural and functional vessel wall properties. It has been shown repeatedly that they are associated with cardiovascular disease and traditional risk factors. $^{1-5}$ Ambulatory blood pressure measurements (ABPM) have a higher reproducibility than office blood pressure measurements and predict cardiovascular risk over and above conventional blood pressure measurements. ${ }^{6}$ The presence of cardiovascular disease, however, can also be predicted relatively effectively with multivariable risk models composed of traditional risk factors. ${ }^{7}$ Therefore, in the evaluation of a novel risk marker, the additive effect should be confirmed in the presence of other established risk factors in a given population to determine its true value. Few studies have addressed this in populations at high risk for cardiovascular disease.

The objective of the present study was to evaluate the additional value of measuring IMT, PWV and ABPM in a cohort of patients at high risk for cardiovascular disease. All patients in this report underwent renal angiography because of the clinical suspicion of renal artery stenosis. Previously, we developed a risk score for the diagnosis of renal artery stenosis in this group of patients. [van Onna M, Nelemans PJ, Kessels AGH, Kroon AA, Flobbe K, de Haan M, de Leeuw PW. The predictive value of clinical clues for the diagnosis of renal artery stenosis in clinical practice. Submitted for publication. 2007] We considered recognized risk factors for cardiovascular disease as well as established clinical clues for renovascular hypertension as candidate predictors. Six predictors were identified: age, office pulse pressure, smoking, onset of hypertension within two years, extra-renal atherosclerosis and a difference in kidney length exceeding $10 \%$ (as measured by renal ultrasound). This model discriminated well between patients with and without renal artery stenosis in a group of consecutive patients who underwent renal angiography. Yet, we hypothesized that the precision of predictions could be improved by adding IMT, PWV and ABPM because they might provide a more refined measure of extra-renal atherosclerosis and pulse pressure respectively.

\section{Materials and methods}

\section{Patients}

As of April 1999, all patients referred to our outpatient clinic for work-up of their hypertension, undergo carotid ultrasonography, PWV measurement and ABPM after their antihypertensive medication has been discontinued for three weeks. In a subset of these patients renal angiography is performed eventually. Previously, we have studied the clinical characteristics of our angiography patients and constructed a prediction 
model for the diagnosis of renal artery stenosis. [van Onna M, Nelemans PJ, Kessels $A G H$, Kroon AA, Flobbe $K$, de Haan $M$, de Leeuw PW. The predictive value of established clinical clues for the diagnosis of renal artery stenosis in clinical practice. Submitted for publication. 2007] In short, patients were judged eligible for that study if they were between 18 and 75 years of age, had a diastolic blood pressure equal to or exceeding $95 \mathrm{mmHg}$, and had one or more clinical characteristics suggestive of the presence of renovascular hypertension ${ }^{8,9}$ and/or signs commonly associated with renal artery disease. Suspicion of renovascular hypertension had to have been the main indication for angiography. We retrieved clinical files from these patients and collected data using standardized forms. The present report comprises all subjects that contributed to the prediction model and underwent angiography between April 1999 and October 2003. Only examinations of B-mode ultrasonography, aortic pulse wave velocity and ambulatory blood pressure measurements that were performed within a 6 month-period before renal angiography were considered.

\section{B-mode ultrasonography}

B-mode ultrasound examination is performed with an ultrasound apparatus with a transducer frequency of $7.5 \mathrm{Mhz}$ and a three-lead ECG modality (Sigma 44, Kontron Instruments). The IMT of the right and left common carotid artery (CCA) is measured with the patient in recumbent position. The CCA is scanned longitudinally and the lumen-intima and the media-adventitia interfaces of the far wall are visualized. We obtain an image in the end-diastolic phase of the cardiac cycle (R-wave on the ECG) and store it on a personal computer. Before June 2001 only one image per side was acquired, thereafter, we have depicted the CCA in four angles per side $\left(90^{\circ}, 120^{\circ}, 150^{\circ}\right.$, $180^{\circ}$ on the right side and $270^{\circ}, 240^{\circ}, 210^{\circ}$, and $180^{\circ}$ on the left side respectively) and thus four images per side are obtained. On each image, the mean distance between the lumen-intima and the media-adventitia interfaces is measured using an automated edge detection algorithm (M'Ath 2.0.1; Metris) in the ten $\mathrm{mm}$ segment proximal to the bifurcation of the carotid artery. For the current analysis, we averaged mean IMT of the left and right CCA.

\section{Pulse wave velocity}

Carotid-femoral PWV was measured by an automated device (Complior, Colson) after at least fifteen minutes rest with the patient in supine position. With the use of this apparatus, the technician obtains transcutaneously waveforms at the right common carotid artery and at the right femoral artery and the time delay (dt) is assessed between the feet of the waveforms. At least ten measurements with a good upstroke are acquired. The distance (D) between the recording sites is measured. The PWV is calculated automatically by the device as D/dt. 


\section{Ambulatory blood pressure monitoring}

Ambulatory blood pressure is measured with either Spacelabs 90207, Spacelabs 90217-b (Spacelabs Medical, Inc.) or the TM-2430 (A \& D Engineering, Inc.) during 24 hours, with the cuff applied to the non-dominant arm. Blood pressure is measured every fifteen minutes during daytime (07:00 a.m. -11.00 p.m.) and every 30 minutes during night hours (11:00 p.m. - 07:00 a.m.) Patients are instructed to adhere to their normal daily activities and sleeping hours.

\section{Statistical analysis}

Since deleting cases with missing data potentially causes bias, we replaced missing values by multiple imputation. Multiple imputation involves imputing values for each missing item and creating $\mathrm{m}$ completed data sets. Across these completed data sets, the observed values are the same, but the missing values are filled in with different imputations to reflect uncertainty. For the purpose of imputation, we selected all variables that were intended to enter analyses, and factors that were related to the occurrence of missing data. ${ }^{10}$ We created $\mathrm{m}=20$ datasets with an ExpectationMaximization algorithm with importance sampling. ${ }^{11}$

We investigated the relationships between IMT, ABPM and PWV with bivariate correlation analysis according to Pearson. Differences between the patient groups were tested with a two-sided t-tests or Chi-Square-tests. We explored the predictive ability of IMT, PWV and ABPM for the diagnosis of renal artery stenosis with univariable logistic regression analysis. We tested whether IMT, abdominal PWV and ABPM improved the prediction of renal artery stenosis by use of multivariable logistic regression analysis. In order to do so, we produced five models: 1. IMT added to the variables of the prediction model, 2. PWV added to the variables of the prediction model, 3. Ambulatory PP instead of Office PP in the model 4. IMT, PWV and ABPM added to the variables of the prediction model. 5. IMT and PWV added to the variables of the prediction model. The additive value was evaluated by the change in the -2 Log Likelihood and the (change

in) the area under the curve of the receiver operating characteristic curve (AUC). ${ }^{12,13}$ Analyses were performed with SPSS 11.5 , StataSE 8.0 and Amelia. ${ }^{14}$

\section{Results}

Between April 1999 and October 2003, 294 eligible patients had undergone renal angiography. Results of IMT, PWV and ABPM within the 6 months before angiography, were not available in respectively $12 \%, 29 \%$ and $6 \%$. For various reasons, some people underwent investigations while still being on antihypertensive medication; since this will interfere with the results of PWV and ABPM, these measurements were not further considered (22\%). Angiography showed abnormalities in 79 patients of whom 29 had 
$<50 \%$ atherosclerotic renal artery disease, 38 atherosclerotic renal artery stenosis of $50 \%$ or more, eleven patients fibromuscular dysplasia and one a combination of atherosclerotic and fibromuscular abnormalities. A patient was considered to have significant renal artery stenosis when the angiogram showed fibromuscular dysplasia or $\geq 50 \%$ luminal reduction in case of atherosclerotic disease. Thus, analyses were performed on 50 patients with and 244 without renal artery stenosis.

Table 3.1 shows characteristics of patients with and without renal artery stenosis. Patients with renal artery stenosis presented with a worse cardiovascular risk profile: they were older, had a higher systolic and a lower diastolic blood pressure, a higher serum creatinine and had more often wide-spread atherosclerosis. Table 3.1 also shows that patients with renal artery stenosis had a higher IMT, a higher aortic PWV and higher ambulatory pulse pressure. Noticeably, ambulatory blood pressure measurements were performed while patients were off blood-pressure lowering drugs as opposed to the office measurements which were taken while patients used their medication. Still, ambulatory blood pressure measurements were lower in both patients with and without renal artery stenosis.

Table 3.1 Patient characteristics

\begin{tabular}{lccc} 
Patient characteristic & $\begin{array}{c}\text { No abnormalities } \\
(\mathrm{n}=244)\end{array}$ & $\begin{array}{c}\text { Renal artery stenosis } \\
(\mathrm{n}=50)\end{array}$ & $p$ \\
\hline Age (years) & $53 \pm 12$ & $58 \pm 11$ & 0.002 \\
Male gender $(\%)$ & 57 & 58 & 0.851 \\
Body mass index $\left(\mathrm{kg} / \mathrm{m}^{2}\right)$ & $28 \pm 5$ & $26 \pm 4$ & 0.004 \\
Office systolic blood pressure $(\mathrm{mmHg})$ & $171 \pm 28$ & $178 \pm 20$ & 0.035 \\
Office diastolic blood pressure $(\mathrm{mmHg})$ & $100 \pm 16$ & $95 \pm 12$ & 0.041 \\
Office pulse pressure $(\mathrm{mmHg})$ & $71 \pm 23$ & $83 \pm 20$ & 0.001 \\
Hypercholesterolemia $(\%)$ & 32 & 46 & 0.057 \\
Diabetes mellitus $(\%)$ & 11 & 10 & 0.891 \\
Smoking $(\%)$ & 63 & 88 & 0.001 \\
Creatinine $(\mu$ mol/l) & $100 \pm 37$ & $113 \pm 37$ & 0.029 \\
Kidney volume difference $(\%)$ & 15 & 32 & 0.008 \\
Recent onset hypertension $(\%)$ & 28 & 44 & 0.043 \\
Extra-renal atherosclerosis & & & $<0.0001$ \\
$\quad$ site $(\%)$ & 22 & 28 & \\
2 sites or more $(\%)$ & 9 & 30 & \\
Ambulatory systolic blood pressure $(\mathrm{mmHg})$ & $165 \pm 23$ & $168 \pm 17$ & 0.484 \\
Ambulatory diastolic blood pressure $(\mathrm{mmHg})$ & $103 \pm 15$ & $98 \pm 12$ & 0.057 \\
Ambulatory pulse pressure $(\mathrm{mmHg})$ & $63 \pm 16$ & $70 \pm 14$ & 0.013 \\
Intima media thickness $(\mathrm{mm})$ & $0.68 \pm 0.12$ & $0.73 \pm 0.11$ & 0.012 \\
Aortic pulse wave velocity $(\mathrm{m} / \mathrm{s})$ & $13.7 \pm 3.8$ & $15.6 \pm 4.7$ & 0.007
\end{tabular}

Correlations between ABPM, IMT and PWV are depicted in Table 3.2. Of the blood pressure indices, pulse pressure correlated best with IMT and PWV with correlation coefficients of 0.36 and 0.46 respectively ( $p<0.0001$ for both). IMT and PWV were also statistically significantly correlated with each other $(r=0.38, p<0.0001)$. 
Table 3.2 Matrix of correlations between mean 24-hour ambulatory blood pressure, intima media thickness of the common carotid artery and aortic pulse wave velocity in 144 patients who underwent all three investigations.

\begin{tabular}{llllll} 
Test & aDBP & aPP & aMAP & IMT & PWV \\
\hline aSBP & $0.77^{\mathrm{c}}$ & $0.75^{\mathrm{c}}$ & $0.93^{\mathrm{c}}$ & $0.23^{\mathrm{b}}$ & $0.35^{\mathrm{c}}$ \\
aDBP & & 0.16 & $0.94^{\mathrm{c}}$ & 0.00 & 0.08 \\
aPP & & & $0.46^{\mathrm{c}}$ & $0.36^{\mathrm{c}}$ & $0.46^{\mathrm{c}}$ \\
aMAP & & & 0.13 & $0.22^{\mathrm{b}}$ \\
IMT & & & & & $0.38^{\mathrm{c}}$
\end{tabular}

${ }^{a} p<0.05,{ }^{b} p<0.01,{ }^{c} p<0.001$ aSBP denotes ambulatory systolic blood pressure, aDBP ambulatory diastolic blood pressure, aPP ambulatory pulse pressure, aMAP ambulatory mean arterial pressure, IMT intima media thickness and PWV aortic pulse wave velocity.

Univariable analysis of predictors with renal artery stenosis are presented in Table 3.3. Besides the predictors we identified in previous research (age, pulse pressure, smoking behaviour, length difference between the kidneys, onset of hypertension within two years and extra-renal atherosclerosis), also ambulatory PP, IMT and aortic PWV were predictors of renal artery stenosis. Univariable diagnostic odds ratio's for 24-hour ambulatory blood pressure and office blood pressure were similar.

Table 3.3 Multivariable analysis of original prediction model and suppplemented models.

\begin{tabular}{|c|c|c|c|c|}
\hline \multirow[t]{3}{*}{ Predictor } & \multirow{3}{*}{$\begin{array}{l}\text { Univariable } \\
\text { OR }(95 \%-\mathrm{Cl})\end{array}$} & \multicolumn{3}{|c|}{ Multivariable } \\
\hline & & Model 1 & Model 2 & Model 3 \\
\hline & & OR $(95 \%-\mathrm{Cl})$ & OR $(95 \%-\mathrm{Cl})$ & OR $(95 \%-\mathrm{Cl})$ \\
\hline Age (per 10 years) & $1.55(1.17-2.04)$ & $1.35(0.96-1.89)$ & $1.40(1.00-1.95)$ & $1.20(0.81-1.78)$ \\
\hline Office pulse pressure (per $10 \mathrm{mmHg}$ ) & $1.25(1.09-1.38)$ & $1.18(1.01-1.39)$ & - & $1.16(0.99-1.37)$ \\
\hline Smoking & $4.29(1.76-10.45)$ & $3.61(1.41-9.28)$ & $3.66(1.42-9.40)$ & $3.61(1.38-9.48)$ \\
\hline Kidney volume difference & $2.64(1.32-5.26)$ & $2.69(1.24-5.85)$ & $2.94(1.33-6.50)$ & $2.73(1.24-6.01)$ \\
\hline Recent onset hypertension & $1.92(1.03-3.57)$ & $2.51(1.23-5.11)$ & $2.63(1.28-5.41)$ & $2.49(1.21-5.08)$ \\
\hline Extra-renal atherosclerosis & $2.32(1.56-3.43)$ & $1.61(1.03-2.51)$ & $1.55(0.98-2.45)$ & $1.56(0.98-2.48)$ \\
\hline Ambulatory pulse pressure (per $10 \mathrm{mmHg}$ ) & $1.26(1.05-1.45)$ & - & $1.22(0.97-1.54)$ & - \\
\hline Intima media thickness (per $0.1 \mathrm{~mm}$ ) & $1.40(1.07-1.67)$ & - & - & $1.14(0.80-1.42)$ \\
\hline Aortic pulse wave velocity & $1.11(1.04-1.20)$ & - & - & $1.04(0.57-3.98)$ \\
\hline Area under the ROC-curve & & $0.78(0.71-0.84)$ & $0.77(0.70-0.84)$ & $0.78(0.72-0.85)$ \\
\hline
\end{tabular}

Multivariable analysis of the original prediction model (model 1 ) and of the prediction model when office pulse pressure was replaced by ambulatory pulse pressure (model 2) and when supplemented by intima media thickness and aortic pulse wave velocity (model 3).

Table 3.3 shows that the predictive ability of most univariable predictors was attenuated in multivariable analysis. The AUC of the original model was 0.78 (0.71-0.84) (Table 3.3, Model 1). When either ambulatory pulse pressure or IMT or PWV was added, the predictive ability did not change (AUC was 0.77 (0.70-0.84), $0.78(0.71-0.85)$, and 0.78 (0.71-0.85) respectively (Table 3.3, Model 2)) and the same was true when both IMT and PWV were added (AUC $0.78(0.72-0.85)$ ). (Table 3.3, Model 3). The addition of IMT and PWV did not improve model fit (change of the -2 Log Likelihood of $-1.58, p>0.05$ ). 


\section{Discussion}

We have demonstrated that intima media thickness, pulse wave velocity and ambulatory pulse pressure were interrelated in a population at high risk for cardiovascular disease. Yet, since the correlation coefficients did not exceed 0.5 , their determinants are presumably, at least in part, different. All three additional investigations were associated with renal artery stenosis, but their contribution to a correct diagnosis of renal artery stenosis was negligible in the presence of other risk predictors.

Our findings confirm the results of previous evaluations of the additional value of structural and functional vessel wall properties in the diagnosis of cardiovascular disease in both high ${ }^{15,16}$ and low risk populations. ${ }^{1,17-19}$ Herrington et al. ${ }^{15}$ studied the predictive value of lower extremity arterial compliance in patients suspected of coronary artery stenosis. When added to established risk factors like age, sex, tobacco use, (the presence of) hypertension, hyperlipidemia, diabetes mellitus and obesity, the AUC changed from 0.73 to 0.74 . Held et al. ${ }^{16}$ studied a cohort of patients with stable angina pectoris and found that, whereas IMT predicted the combined endpoint of cardiovascular death, myocardial infarction and cardiac revascularization, it did not when classical risk factors were accounted for. The investigators of the Atherosclerosis Risk in Communities (ARIC) study ${ }^{17}$ investigated incident coronary heart disease in the general population, subdivided according to race (black or white) and gender. On average, the AUC rose with 0.1 when IMT was added to a model containing age, cholesterol blood pressure, smoking behavior, antihypertensive medication and diabetes. In the Rotterdam study ${ }^{18}$ the AUC for the predictions of incident myocardial infarction and stroke in the general population rose from 0.72 to 0.75 when IMT was added. Störk et al. ${ }^{19}$ found that the AUC for prediction of incident cardiovascular mortality in independently living men over 70 years, rose from 0.71 to 0.77 when both carotid plaque score and arterial stiffness were added to a model containing age, medical history, medication-use, body mass index, blood pressure and lipid profile. The improvement of the AUC when carotid stiffness was added to a model already containing carotid plaque score was small ( 0.75 to 0.77 ), IMT did not improve the predictive ability. IMT independently predicted incident myocardial infarction and stroke in the Cardiovascular Health Study. ${ }^{1}$ The additional predictive value, however, was not quantified by means of change in AUC. The multivariable OR of an increase of the maximum common carotid IMT with $0.20 \mathrm{~mm}$ was, however, only 1.27 and this may be comparable to our results although we measured mean IMT and not maximum IMT; we found an multivariable OR of 1.14 per $0.10 \mathrm{~mm}$ increase which is equivalent to an OR of 1.29 per $0.20 \mathrm{~mm}$ increase.

The European Society of Hypertension and the European Society of Cardiology have recommended the measurement of IMT in patients with hypertension to improve risk stratification in their guidelines for the management of arterial hypertension. ${ }^{20}$ They 
considered pulse wave velocity of importance, but they acknowledged that it should be tested further to establish the predictive value. Our results and results from previous studies do not sustain the guideline on this point. The presence of cardiovascular disease can be predicted relatively effectively with multivariable risk models composed of traditional risk factors: in diverse populations with diverse outcome measures the average AUC is about 0.72 in literature. ${ }^{15-19}$ and in our model, in which risk factors specific for renal artery stenosis were considered, the AUC was 0.78. IMT and arterial stiffness measurements did not increase the predictive ability substantially in any of these studies. Therefore, our results and results from previous studies do not support the use of IMT and PWV for risk stratification of an individual patient.

Several issues should be considered in order to fully appreciate our findings. First, the outcome under study was renal artery stenosis which was caused by atherosclerosis in $78 \%$ and by fibromuscular dysplasia in $22 \%$ of the patients. Whereas PWV and IMT have been associated with atherosclerotic disease at multiple sites in numerous studies, to our knowledge only one such report is available in patients with renal fibromuscular dysplasia without carotid artery involvement. ${ }^{21}$ That study demonstrated an association of both common carotid intima media thickness and carotid distensibility with angiographically documented renal artery fibromuscular dysplasia. Still, this should be kept in mind when comparing our data with studies that used only atherosclerotic manifestations like myocardial infarction and stroke as outcome measures. Secondly, a relatively high number of patients did not undergo all three additional investigations. Yet, we dealt with these missing values using state-of-the-art statistical techniques that would be $98 \%$ efficient even when $50 \%$ of the entire dataset would have been missing. ${ }^{22}$ Thirdly, our evaluation is confined to the predictive value of IMT, PWV and ABPM. The value of $A B P M$ is beyond dispute in measuring the "actual blood pressure" for the diagnosis of hypertension and evaluation of treatment. IMT is a valuable intermediate outcome measure that may increase efficiency and feasibility of randomized clinical trials. $^{23}$ For the individual patient, however, the potential value of IMT and PWV assumes that they parallel risk on cardiovascular disease and that favorable changes in these measures indicate a reduction in risk, which has not been proven yet. ${ }^{24}$

\section{Conclusions}

Our results indicate that for the prediction of the presence of renal artery stenosis, more sophisticated measurements (ABP, IMT, PWV) do not perform better than rather crude measurements (anamnesis, medical history, physical examination, office blood pressure). 


\section{References}

1. O'Leary DH, Polak JF, Kronmal RA, Manolio TA, Burke GL, Wolfson SK, Jr. Carotid-artery intima and media thickness as a risk factor for myocardial infarction and stroke in older adults. Cardiovascular Health Study Collaborative Research Group. $N$ Engl J Med. 1999;340:14-22.

2. Raitakari OT, Juonala M, Kahonen M, Taittonen L, Laitinen T, Maki-Torkko N, Jarvisalo MJ, Uhari M, Jokinen E, Ronnemaa T, Akerblom HK, Viikari JS. Cardiovascular risk factors in childhood and carotid artery intima-media thickness in adulthood: the Cardiovascular Risk in Young Finns Study. Jama. 2003;290:2277-83.

3. Laurent S, Boutouyrie P, Asmar R, Gautier I, Laloux B, Guize L, Ducimetiere P, Benetos A. Aortic stiffness is an independent predictor of all-cause and cardiovascular mortality in hypertensive patients. Hypertension. 2001;37:1236-41.

4. Blacher J, Asmar R, Djane S, London GM, Safar ME. Aortic pulse wave velocity as a marker of cardiovascular risk in hypertensive patients. Hypertension. 1999;33:1111-7.

5. Simons PC, Algra A, Bots ML, Grobbee DE, van der Graaf Y. Common carotid intima-media thickness and arterial stiffness: indicators of cardiovascular risk in high-risk patients. The SMART Study (Second Manifestations of ARTerial disease). Circulation. 1999;100:951-7.

6. Staessen JA, Thijs L, Fagard R, O'Brien ET, Clement D, de Leeuw PW, Mancia G, Nachev C, Palatini P, Parati G, Tuomilehto J, Webster J. Predicting cardiovascular risk using conventional vs ambulatory blood pressure in older patients with systolic hypertension. Systolic Hypertension in Europe Trial Investigators. Jama. 1999;282:539-46.

7. Grundy SM, D'Agostino Sr RB, Mosca L, Burke GL, Wilson PW, Rader DJ, Cleeman JI, Roccella EJ, Cutler JA, Friedman LM. Cardiovascular risk assessment based on US cohort studies: findings from a National Heart, Lung, and Blood institute workshop. Circulation. 2001;104:491-6.

8. Mann SJ, Pickering TG. Detection of renovascular hypertension. State of the art: 1992. Ann Intern Med. 1992;117:845-53.

9. Detection, evaluation, and treatment of renovascular hypertension. Final report. Working Group on Renovascular Hypertension. Arch Intern Med. 1987;147:820-9.

10. Van Buuren S, Boshuizen HC, Knook DL. Multiple imputation of missing blood pressure covariates in survival analysis. Stat Med. 1999;18:681-94.

11. King G, Honaker J, Joseph A, Scheve K. Analyzing incomplete political science data: an alternative algorithm for multiple imputation. American Political Science Review. 2001;95: 49-69.

12. Hanley JA, McNeil BJ. The meaning and use of the area under a receiver operating characteristic (ROC) curve. Radiology. 1982;143:29-36.

13. Hanley JA, McNeil BJ. A method of comparing the areas under receiver operating characteristic curves derived from the same cases. Radiology. 1983;148:839-43.

14. Honaker J, Joseph A, King G, Scheve K, Singh N. Amelia: a program for missing data (Windows version) Cambridge, MA: Harvard University ; 2001. Available as freeware from http://GKing.Harvard.edu. Accessed 10 Sept 2004.

15. Herrington DM, Kesler K, Reiber JC, Davis W, Brown WV, Helms R, Mallon SM, Raines J. Arterial compliance adds to conventional risk factors for prediction of angiographic coronary artery disease. Am Heart J. 2003;146:662-7.

16. Held C, Hjemdahl P, Eriksson SV, Bjorkander I, Forslund L, Rehnqvist N. Prognostic implications of intima-media thickness and plaques in the carotid and femoral arteries in patients with stable angina pectoris. Eur Heart J. 2001;22:62-72.

17. Chambless LE, Folsom AR, Sharrett AR, Sorlie P, Couper D, Szklo M, Nieto FJ. Coronary heart disease risk prediction in the Atherosclerosis Risk in Communities (ARIC) study. J Clin Epidemiol. 2003;56:880-90.

18. del Sol AI, Moons KG, Hollander M, Hofman A, Koudstaal PJ, Grobbee DE, Breteler MM, Witteman JC, Bots ML. Is carotid intima-media thickness useful in cardiovascular disease risk assessment? The Rotterdam Study. Stroke. 2001;32:1532-8. 
19. Störk S, van den Beld AW, von Schacky C, Angermann CE, Lamberts SW, Grobbee DE, Bots ML. Carotid artery plaque burden, stiffness, and mortality risk in elderly men: a prospective, population-based cohort study. Circulation. 2004;110:344-8.

20. 2003 European Society of Hypertension-European Society of Cardiology guidelines for the management of arterial hypertension. J Hypertens. 2003;21:1011-53.

21. Boutouyrie P, Gimenez-Roqueplo AP, Fine E, Laloux B, Fiquet-Kempf B, Plouin PF, Jeunemaitre $X$, Laurent $S$. Evidence for carotid and radial artery wall subclinical lesions in renal fibromuscular dysplasia. J Hypertens. 2003;21:2287-95.

22. Schafer JL, Graham JW. Missing data: our view of the state of the art. Psychol Methods. 2002;7:147-77.

23. Zanchetti A, Bond MG, Hennig M, Neiss A, Mancia G, Dal Palu C, Hansson L, Magnani B, Rahn KH, Reid JL, Rodicio J, Safar M, Eckes L, Rizzini P. Calcium antagonist lacidipine slows down progression of asymptomatic carotid atherosclerosis: principal results of the European Lacidipine Study on Atherosclerosis (ELSA), a randomized, double-blind, long-term trial. Circulation. 2002;106:2422-7.

24. Bots ML, Dijk JM, Oren A, Grobbee DE. Carotid intima-media thickness, arterial stiffness and risk of cardiovascular disease: current evidence. J Hypertens. 2002;20:2317-25. 



\section{Chapter 4}

Genetic risk of atherosclerotic renal artery

disease: the candidate gene approach in a renal angiography cohort

Marieke van Onna, Abraham A. Kroon, Alfons J.H.M. Houben, Derk Koster, Maurice P.A. Zeegers, Léon H.G. Henskens, Arian W. Plat, Henri E.J.H. Stoffers, Peter W. de Leeuw

Hypertension. 2004;44:448-453 


\section{Abstract}

It is largely unknown to what extent genetic abnormalities contribute to the development of atherosclerotic renal artery disease. Among the potential candidate genes, those of the reninangiotensin system and the endothelial nitric oxide synthase (eNOS) rank high, because of their importance in the atherosclerotic process. We investigated the association of polymorphisms in these genes (the angiotensinogen Met235Thr, the angiotensin converting enzyme insertion/deletion, the angiotensin II type 1 receptor A1166C and the eNOS Glu298Asp) with the presence or absence of atherosclerotic renovascular disease, in 456 consecutive hypertensive patients referred for renal angiography on the suspicion of renovascular hypertension. Nondiseased normotensive $(n=200)$ and hypertensive $(n=154)$ patients from a family practice served as external controls. Renal artery disease was present in $30 \%$ of our angiography group. The Aspallele of the endothelial nitric oxide synthase Glu298Asp polymorphism was associated with atherosclerotic renal artery stenosis with an odds ratio (95\% confidence interval) of 1.44 (1.00-2.09) versus hypertensives with angiographically-proven patent arteries, of 1.89 (1.24-2.87) versus hypertensive family practice controls and of 2.09 (1.29-3.38) versus normotensive family practice controls. However, this allele differed also significantly between patients with patent renal arteries and both normotensive and hypertensive controls. No differences were found with respect to the other genetic polymorphisms. 


\section{Introduction}

Atherosclerotic renal artery stenosis (ARAS) is a relatively common cause of secondary hypertension. ${ }^{1}$ Nowadays, most investigators consider this abnormality to be part of a generalized atherosclerotic complex with an etiological background similar to extrarenal atherosclerosis.

Although it is still largely unknown if and to what extent genetic abnormalities contribute to the development of this disease, polymorphisms in genes of the renin-angiotensin system rank high among the potential candidates. For instance, the D-allele of the insertion/deletion variant of the angiotensin converting enzyme (ACE) gene has been associated with atherosclerotic disease, ${ }^{2,3}$ and it is conceivable that this is true also for ARAS. Another genetic variant that could have a bearing on the renal vasculature is the angiotensin II type 1 receptor $A 1166 C \quad\left(A T_{1} R A{ }^{1166} C\right)$ polymorphism. Patients homozygous for the $\mathrm{C}$-allele of this polymorphism have increased sensitivity to angiotensin $\mathrm{II}^{4}$ and may, in combination with the DD genotype of the ACE gene, run an increased risk of cardiovascular complications. ${ }^{5}$ Finally, the angiotensinogen Met235Thr (AGT Met235Thr) polymorphism is of interest because the Thr-allele of this polymorphism is associated with high plasma levels of AGT and increased responsiveness to angiotensin $11 .{ }^{6}$

In addition to studies of angiotensin II and its precursors, many studies have highlighted the importance of endothelium-derived nitric oxide (NO) in inhibiting atherosclerotic disease and the possibility that impairment of endothelial NO production promotes atherosclerosis. NO is produced by endothelial nitric oxide synthase (eNOS). The gene encoding eNOS has a G to T polymorphism at position 894 leading to substitution of Glu by Asp at codon 298 of the eNOS protein. The mutant allele is associated with a reduced response to the eNOS inhibitor L-NMMA in healthy volunteers, ${ }^{7}$ and may be related to the occurrence of atherosclerotic complications.

Although a few earlier studies have addressed the possible role of candidate genes such as the ones described above in renovascular disease,${ }^{8-11}$ none of these have been performed in patient populations that were primarily selected for their risk of having ARAS. This is important because of the complex, yet unresolved relation between anatomical abnormalities and clinical symptoms of renovascular disease. ${ }^{12}$ In the present study, therefore, we have examined the distribution of the alleles of the abovementioned four candidate genes in a group of patients in whom renovascular hypertension was clinically suspected and who all underwent renal angiography for this reason. 


\section{Methods}

\section{Patients}

Between January 1995 and December 2002, 456 patients referred to our outpatient clinic for evaluation of their hypertension underwent renal angiography. Hypertension was defined as systolic blood pressure above $140 \mathrm{mmHg}$ or diastolic blood pressure above $90 \mathrm{mmHg}$ on at least three occasions. All patients fulfilled one or more of the following criteria: persistent elevation of blood pressure despite the use of two or more antihypertensive drugs, accelerated hypertension, documented atherosclerotic vascular disease in two or more vascular beds, the presence of an abdominal bruit or unexplained impairment of renal function in response to antihypertensive treatment.

Since mid-1996 we ask written permission from all newly referred patients to draw a $3 \mathrm{ml}$ blood sample for genetic analysis, as approved by the local Medical Ethics Committee. Patients who had undergone angiography between January 1995 and June 1996 were sent a letter with the request to visit our laboratory to donate a blood sample. Medical history and risk factor profiles were derived from the clinical files

Two experienced readers evaluated all angiographic films and reported on the site and nature of the aberration and the (maximum) \%-stenosis via standardized forms. If the two estimates of the degree of stenosis differed by more than $10 \%$, a third opinion was decisive. Since there is no consensus on what degree of renal artery narrowing is hemodynamically significant and atheromateous renal artery abnormalities are likely to progress in time, ${ }^{13}$ we divided our study participants into two groups: (1) patent renal arteries and (2) (any grade of) atheromateous renal artery disease. Patients with nonatheromateous renal artery disease (mainly fibromuscular dysplasia) were excluded from analysis.

\section{Control populations}

Hypertensive $(n=154)$ and normotensive $(n=200)$ subjects from the HIPPOCRATES study, which is an ongoing study on cardiovascular risk and genetics in a general practice in Kerkrade, The Netherlands, served as control groups for the angiotensin converting enzyme insertion/deletion, the angiotensin II type 1 receptor $A 1166 \mathrm{C}$ and the endothelial nitric oxide synthase Glu298Asp genotype distributions. The details of the enrollment of the study participants have been published previously. ${ }^{14}$

\section{Genetic analysis}

Genetic analysis was performed as previously described, ${ }^{14}$ Table 4.1 shows the primers that were used. 
Table 4.1 Primers used for detection of ACE I/D, AT1R A ${ }^{1166} \mathrm{C}$, AGT Met235Thr and eNOS Glu298Asp polymorphisms.

\begin{tabular}{ll} 
Polymorphism & Primer \\
\hline ACE I/D & 5'-GATGTGGCCATCACATTCGTCAGAT-3' \\
5'-CTGGAGACCACTCCCATCCTTTCT-3' \\
AT1R A1166C & 5'-GAAGGAGCAAGAGAACATTCGACTG \\
CAGCACTTCACTACCAAATGAGAC-3' & 5'-CTCTGCAGCACTTCACTACCAAATGATCA-3' \\
5'-TTTCTGACATTGTTCTTCGAGCAGCCGT-3' & \\
AGT Met235Thr & 5'-CCGTTTGTGCAGGGCCTGGCTCTCT-3' \\
5'-CAGGGTGCTGTCCACACTGGACCCC-3' \\
eNOS Glu298Asp \\
5'-AACGGTCGCTTCGACGTGCACCCCCT- \\
'GCTGCTGCAGGCCCAGATGGT-3' \\
5'-TGCCCCTGCTGCTGCAGGCCCCAGATTAG-3' \\
5'-TGTGGGATCAGCACCCCCTTGCAGGCCC-3'
\end{tabular}

\section{Statistical analysis}

Statistical significance for differences in quantitative variables was tested by unpaired t-tests or Mann-Whitney tests when appropriate. Allele and genotype frequencies and other qualitative data were analysed using $\chi^{2}$-tests. Hardy-Weinberg equilibrium was tested using standard methods. ${ }^{15}$ Genetic models were further evaluated by logistic regression analysis, in order to "correct" for those variables (confounders), which would alter the interpretation of the relationship between genotype and presence of renal artery abnormalities when not included in the model. ${ }^{16}$ We regarded age, sex, body mass index, pulse pressure and renal function as potential confounders. We choose to regard only pulse pressure (PP) of the blood pressure parameters in our analysis because imputing systolic blood pressure (SBP) and diastolic blood pressure (DBP) as well would inevitably lead to collinearity. (Pearson's correlation coefficients were 0.83 for PP vs. SBP, 0.28 for PP vs. DBP and 0.76 for SBP vs. DBP; $p<0.0001$ for all). We introduced the potential confounders independently into a model that contained genotype and assessed the change of the regression coefficient of the central determinant (genotype). The variable that led to the greatest change was retained in the model, provided that this change was at least $10 \%$. The remaining potential confounders were then introduced again into the new model (containing genotype and the confounder). This was repeated until no more variables had to be retained. Interactions between two polymorphisms were tested in models that included the alleles (risk allele present or not present), the interaction between the two, and confounders when applicable. We assumed that both groups from the family practice contained no subjects with renal artery stenosis. However, previous studies suggest that secondary hypertension is present in about $5 \%$ of hypertensive patients and renovascular hypertension in approximately $1 \% .{ }^{17-20}$ To the best of our knowledge no such data are 
available on the presence of incidental renal artery stenosis in subjects without hypertension, but conceivably, this value is much lower. We tested the robustness of statistically significant results in the hypertensive group by a sensitivity analysis ${ }^{21}$ by modelling odds ratios after deleting $5 \%$ of the subjects in this group with all possible alleles. Data are expressed as mean $\pm S D$ or median (interquartile range) or odds ratio (95\% confidence interval) unless indicated otherwise. A two-sided p-value of less than 0.05 was considered statistically significant. Statistical analyses were performed with SPSS 10.0 for Windows (SPSS Inc).

\section{Results}

\section{Patient characteristics}

Out of the 456 patients who underwent angiography, genotyping was available in 401 $(88 \%)$. The remainder did not differ from the genotyped patients regarding demographics or important potential confounders. Thirty-three of the 401 patients were diagnosed with fibromuscular dysplasia and, therefore, excluded from analysis. Thus, the present report is based upon the remaining 368 subjects. In $109(30 \%)$ of these, atherosclerotic renal artery abnormalities were present, among which 70 (19\%) with a renal arterial lumen reduction of more than $50 \%$.

General characteristics of the four study groups are presented in Table 4.2. As expected, both normotensive and hypertensive controls from the general practice had lower systolic, diastolic and pulse pressures in comparison to the subjects with renal artery stenosis. In addition, they were more often female and had higher BMl's (although not statistically significant in the normotensive group). Normotensive controls were slightly older than the angiography group whereas the hypertensive controls were a bit younger. In the angiography group, patients with renal artery stenosis (ARAS+) presented with a worse cardiovascular risk profile than individuals with non-diseased renal arteries (ARAS-): they were older, more often male, and had a longer history of hypertension $(6(2-17)$ vs. $4(1-11)$ years, $p=0.014)$. In addition they had higher pulse pressure, a lower estimated creatinine clearance $(56 \pm 22$ vs. $87 \pm 32 \mathrm{ml} / \mathrm{min}, p<0.001)$ and were more often hypercholesterolemic $(45 \%$ vs. $29 \%, p=0.003)$ and diabetic $(16 \%$ vs. $9 \%, p=0.043)$. Finally, they had more often suffered from atherosclerotic manifestations in other vascular beds $(43 \%$ vs. $22 \%, p<0.001)$. 
Table 4.2. Clinical characteristics of the study groups.

\begin{tabular}{|c|c|c|c|c|c|c|c|}
\hline \multirow[t]{3}{*}{ Clinical characteristics } & \multicolumn{4}{|c|}{ General practice } & \multicolumn{3}{|c|}{ Angiography } \\
\hline & \multicolumn{2}{|c|}{ normotensive } & \multicolumn{2}{|c|}{ hypertensive } & ARAS- & & ARAS+ \\
\hline & & $\mathrm{p}$ & & $\mathrm{p}$ & \multicolumn{3}{|c|}{$\mathrm{p}$} \\
\hline Age (years) & $63 \pm 12$ & 0.039 & $57 \pm 11$ & 0.034 & $50 \pm 13$ & $<0.001$ & $60 \pm 10$ \\
\hline Sex (male/female) & $95 / 105$ & 0.003 & $72 / 82$ & 0.013 & $137 / 122$ & 0.031 & $71 / 38$ \\
\hline BMI $\left(\mathrm{kg} / \mathrm{m}^{2}\right)$ & $27 \pm 4$ & 0.075 & $29 \pm 6$ & 0.007 & $28 \pm 5$ & $<0.001$ & $26 \pm 4$ \\
\hline $\mathrm{SBP}(\mathrm{mmHg})$ & $133 \pm 15$ & $<0.001$ & $158 \pm 20$ & $<0.001$ & $176 \pm 28$ & 0.015 & $184 \pm 28$ \\
\hline $\mathrm{DBP}(\mathrm{mmHg})$ & $78 \pm 7$ & $<0.001$ & $92 \pm 11$ & $<0.001$ & $105 \pm 16$ & 0.016 & $100 \pm 18$ \\
\hline $\mathrm{PP}(\mathrm{mmHg})$ & $54 \pm 13$ & $<0.001$ & $65 \pm 16$ & $<0.001$ & $71 \pm 21$ & $<0.001$ & $84 \pm 22$ \\
\hline
\end{tabular}

Values are presented as mean $\pm S D$. ARAS denotes atherosclerotic renal artery stenosis, $p$ refers to the $p$-value of the test of the difference with ARAS+, BMI denotes body mass index, SBP systolic blood pressure, DBP diastolic blood pressure.

\section{Genetic analyses}

The distribution of the polymorphisms in the control populations was compatible with the Hardy-Weinberg equilibrium, whereas this was not the case in the angiography group with respect to the eNOS Glu298Asp (only ARAS) and the AGT Met235Thr polymorphisms. (Table 4.3) No differences in either genotype or allele frequencies were found between the normotensive and the hypertensive control group.

Table 4.3 Distribution of genotypes in controls and angiography patients.

\begin{tabular}{|c|c|c|c|c|c|}
\hline \multirow[t]{2}{*}{ Polymorphism } & \multirow[t]{2}{*}{ Genotype/allele } & \multicolumn{2}{|c|}{ General care population n (\%) } & \multicolumn{2}{|c|}{ Angiography n (\%) } \\
\hline & & normotensive & hypertensive & ARAS- & ARAS+ \\
\hline \multirow[t]{5}{*}{ AGT } & Met/Met & - & - & $104(40.2)$ & $38(34.9)$ \\
\hline & Met/Thr & - & - & $147(56.5)$ & $70(64.2)$ \\
\hline & Thr/Thr & - & - & $8(3.1)$ & $1(0.9)$ \\
\hline & $\operatorname{Met}^{a}$ & - & - & $355(69)$ & $146(67)$ \\
\hline & $\operatorname{Thr}^{\mathrm{a}}$ & - & - & $163(32)$ & $72(33)$ \\
\hline \multirow[t]{5}{*}{ ACE } & Ins/Ins & $46(23.3)$ & $42(27.3)$ & $51(19.5)$ & $22(20.2)$ \\
\hline & Ins/Del & $98(49.7)$ & $68(44.2)$ & $132(50.6)$ & $58(53.2)$ \\
\hline & Del/Del & $53(26.9)$ & $44(28.6)$ & $78(29.9)$ & $29(26.6)$ \\
\hline & $\operatorname{lns}^{a}$ & $190(48)$ & $154(49)$ & $234(45)$ & $102(47)$ \\
\hline & $\mathrm{Del}^{\mathrm{a}}$ & $204(52)$ & $156(51)$ & $288(55)$ & $116(53)$ \\
\hline \multirow[t]{5}{*}{$\mathrm{AT}_{1} \mathrm{R}$} & AA & $104(52.5)$ & $79(51.3)$ & $140(53.6)$ & $52(47.7)$ \\
\hline & $A C$ & 77 (38.9) & $65(42.4)$ & $99(37.9)$ & $47(43.1)$ \\
\hline & $\mathrm{CC}$ & $17(8.6)$ & $10(6.5)$ & $22(8.4)$ & $10(9.2)$ \\
\hline & $A^{a}$ & $285(72)$ & $223(72)$ & $379(73)$ & $151(69)$ \\
\hline & $C^{a}$ & $111(28)$ & $85(28)$ & $143(27)$ & $67(31)$ \\
\hline \multirow[t]{5}{*}{ eNOS } & Glu/Glu & $87(43.5)$ & $63(40.9)$ & $104(40.2)$ & $33(30.3)$ \\
\hline & Glu/Asp & $97(48.5)$ & $75(48.7)$ & $94(36.6)$ & $49(45.0)$ \\
\hline & Asp/Asp & $16(8.0)$ & $16(10.4)$ & $61(23.6)$ & $27(24.8)$ \\
\hline & $\mathrm{Glu}^{\mathrm{a}}$ & $271(68)$ & $201(65)$ & $302(58)$ & $115(53)$ \\
\hline & $\mathrm{Asp}^{\mathrm{a}}$ & $129(32)$ & $107(35)$ & $216(42)$ & $103(47)$ \\
\hline
\end{tabular}

${ }^{a} \mathrm{n}$ refers to the number of alleles. In bold genotype distributions that are not in Hardy-Weinberg equilibrium, ARAS denotes atherosclerotic renal artery stenosis. The AGT Met235Thr polymorphism was not investigated in the HIPPOCRATES study, and this information is therefore lacking in these subjects. 


\section{Normotensive controls versus patients with ARAS+}

The distribution of the eNOS Glu298Asp polymorphism was different between normotensive controls and subjects with renal artery disease $\left(\chi^{2}=17.6, p<0.0001\right)$. When the presence of renal artery disease was taken as dependent variable in logistic regression and the Glu/Glu as reference category, the odds ratios of the Glu/Asp and Asp/Asp genotypes were $1.33(0.79-2.26)$ and 4.45 (2.13-9.30) respectively. After correction for pulse pressure, sex and age, the odds ratios were $1.39(0.61-3.16)$ and 5.08 (1.61-16.0) respectively. To allow comparison with data from the angiography group, we also performed analysis of allele frequencies, which yielded comparable results (Table 4.4). The frequency of the ACE ins/del and the ATR1 A1166C polymorphisms did not differ between controls and patients with renal artery stenosis. There were no interactions between the polymorphisms.

Table 4.4 Logistic regression analyses: the association of eNOS Glu298Asp with renal artery disease.

\begin{tabular}{|c|c|c|}
\hline Model & $\begin{array}{l}\text { Univariable analysis } \\
\text { Odds Ratio }(95 \%-\mathrm{Cl})\end{array}$ & $\begin{array}{l}\text { Multivariable analysis } \\
\text { Odds Ratio }(95 \%-\mathrm{Cl})\end{array}$ \\
\hline \multicolumn{3}{|l|}{ Normotensive controls versus ARAS+ } \\
\hline $\begin{array}{l}\text { eNOS (Asp-allele) })^{a} \\
\text { Pulse pressure }^{b}\end{array}$ & $1.82(1.28-2.58)$ & $\begin{array}{l}2.09(1.29-3.38) \\
2.97(2.49-3.55)\end{array}$ \\
\hline \multicolumn{3}{|l|}{ Hypertensive controls versus ARAS+ } \\
\hline $\begin{array}{l}\text { eNOS (Asp-allele) } \\
\text { Pulse pressure }^{\mathrm{b}}\end{array}$ & $1.68(1.18-2.40)$ & $\begin{array}{l}1.89(1.24-2.87) \\
1.73(1.54-1.94)\end{array}$ \\
\hline \multicolumn{3}{|l|}{ ARAS- versus ARAS+ } \\
\hline $\begin{array}{l}\text { eNOS (Asp-allele) }{ }^{a} \\
\text { Creatinine clearance }^{b}\end{array}$ & $1.25(0.91-1.72)$ & $\begin{array}{l}1.44(1.00-2.09) \\
0.67(0.62-0.72)\end{array}$ \\
\hline
\end{tabular}

a Odds ratio compared to eNOS Glu 298 (=reference category); ${ }^{\text {b }}$ Per 10 units ARAS- refers to patients with angiographically proven patent arteries, ARAS+ to those with renal artery stenosis.

\section{Hypertensive controls versus patients with ARAS+}

Genotype frequencies of the eNOS Glu298Asp polymorphism were different between hypertensive controls and subjects with renal artery disease $\left(\chi^{2}=10.2, p=0.006\right)$. When the presence of renal artery disease was taken as dependent variable in logistic regression and the Glu/Glu as reference category, the odds ratios of the Glu/Asp and Asp/Asp genotypes were $1.22(0.69-2.18)$ and 2.88 (1.33-6.23) respectively. After correction for pulse pressure and sex, the odds ratios were $1.29(0.66-2.53)$ and 4.17 (1.73-10.05), respectively. Analysis of allele frequencies yielded comparable results (Table 4.4). This association remained even when one would assume that $5 \%$ of the hypertensive controls had incidental renal artery stenosis (and therefore would have had to be excluded from analyses). For details, please see Appendix. The frequencies of the 
ACE ins/del and the ATR1 A1166C polymorphisms did not differ between controls and patients with renal artery stenosis. There were no interactions between the polymorphisms.

\section{Angiography group: ARAS- versus ARAS+}

The genotype frequency distribution was not different with regard to the polymorphisms under study. Because there was no Hardy-Weinberg equilibrium with respect to the eNOS Glu298Asp and the AGT Met235Thr polymorphisms, we also tested differences in allele frequency. Again, this did not yield significant results. However, logistic regression analysis showed that the Asp-allele had an odds ratio of 1.44 in comparison to the Glu-allele on ARAS, conditionally on estimated creatinine clearance (Table 4.4). There were no interactions between the polymorphisms.

\section{ARAS- versus controls}

When the ARAS- group was contrasted with the two general care control groups, the Asp-allele appeared to be significantly more frequent in ARAS- than in both control groups (Table 4.5).

Table 4.5 Logistic regression analyses: the association of eNOS Glu298Asp with differences between the control groups.

\begin{tabular}{|c|c|c|}
\hline Model & $\begin{array}{l}\text { Univariable analysis } \\
\text { Odds Ratio }(95 \%-\mathrm{Cl})\end{array}$ & $\begin{array}{l}\text { Multivariable analysis } \\
\text { Odds Ratio }(95 \%-\mathrm{Cl})\end{array}$ \\
\hline \multicolumn{3}{|c|}{ Normotensive vs hypertensive controls } \\
\hline $\begin{array}{l}\text { eNOS (Asp-allele) }{ }^{a} \\
\text { Pulse pressure }^{b}\end{array}$ & $1.12(0.82-1.53)$ & $\begin{array}{l}1.33(1.03-1.71) \\
1.71(1.52-1.94)\end{array}$ \\
\hline \multicolumn{3}{|c|}{ Normotensive controls versus ARAS- } \\
\hline $\begin{array}{l}\text { eNOS (Asp-allele) } \\
\text { Pulse pressure }^{\mathrm{b}} \\
\text { Age }^{\mathrm{b}}\end{array}$ & $1.50(1.14-1.97)$ & $\begin{array}{l}1.58(1.22-2.06) \\
2.59(2.23-3.00) \\
0.29(0.24-0.34)\end{array}$ \\
\hline \multicolumn{3}{|c|}{ Hypertensive controls versus ARAS- } \\
\hline $\begin{array}{l}\text { eNOS (Asp-allele) })^{a} \\
\text { Pulse pressure }^{b}\end{array}$ & $1.34(1.00-1.80)$ & $\begin{array}{l}1.30(1.06-1.59) \\
1.19(1.10-1.29)\end{array}$ \\
\hline
\end{tabular}

a Odds ratio compared to eNOS Glu 298 (=reference category); ${ }^{\text {b }}$ Per 10 units; ARAS- refers to patients with angiographically proven patent arteries.

\section{Discussion}

The objective of our study was to investigate the association of several candidate genes with atherosclerotic renal artery stenosis (ARAS) in a population of patients with hypertension and clinical clues suggesting the presence of renal artery stenosis. All 
patients were subjected to angiography and the prevalence of renal artery abnormalities in this group was $30 \%$. We found that patients with ARAS (ARAS+) were more often carrier of the Asp-allele with regard to the eNOS Glu298Asp polymorphism. Since this association only just reached statistical significance, we sought for additional evidence that the association was a genuine one. Therefore, we compared ARAS+ to two sets of non-diseased controls: one of hypertensive and one of normotensive subjects which were drawn from a family practice. Again, the Asp-allele had a significantly higher prevalence in patients with renovascular disease. Nevertheless, even the use of these control groups is not free of assumptions. The prevalence of renal artery stenosis among hypertensive patients in population samples is, indeed, reported to be low, around $1 \%{ }^{17-20}$ Still, we remain uncertain about the true prevalence of ARAS in controls. However, model analysis showed that our results are robust to a low prevalence of this condition in the control group. Furthermore, the University Hospital Maastricht is the nearest academic centre, but it is not the hospital closest at hand. This implies that patients from Kerkrade with a moderate severe clinical picture have a lower probability of undergoing renal angiography in Maastricht than patients with similar complaints from the Maastricht region, and will, therefore, be underrepresented in this study. Yet, we have no clues that our current controls from a general care practice are any different from a random selection of subjects from a general practice in Maastricht.

Positive findings in an association study can be attributed to three situations. First, the allele itself directly affects the expression of the phenotype. Second, the allele is in linkage disequilibrium with another allele that is responsible for the effect. Third, the association is brought about by confounding or selection bias. ${ }^{22}$ Whereas the first two situations do not hamper the use of a polymorphism for predicting the presence of a condition, the third obviously does. One of the major concerns in this respect is the presence of selection bias. The routine use of the Hardy-Weinberg equilibrium test has been advocated in order to reduce false-positive findings. ${ }^{23}$ Hardy-Weinberg equilibrium was absent in ARAS-. We can only speculate about the explanation for this phenomenon, but it is very likely to be related to the fact that these patients are not truly healthy controls. Especially the underrepresentation of heterozygotes in ARASsuggests that the presence or absence of the Asp-allele itself may have influenced the chance for an individual of being selected for angiography according to our criteria.

Our finding of an association between the presence of renal artery stenosis and the eNOS Glu298Asp polymorphism should be interpreted cautiously since the prevalence of Asp-carriers was also higher in the ARAS- group than in the control groups. This implies that the ARAS- group differed from controls by an (unmeasured) variable (or possibly variables) that coincides with the differences in genotype distribution of the eNOS Glu298Asp polymorphism. Although we cannot rule out that the mutant gene polymorphism is associated with more severe hypertension, as both ARAS- and ARAS+ patients had substantially higher blood pressure than controls, this option does not fully 
explain the data either since we accounted for blood pressure differences by logistic regression analysis. Although the introduction of pulse pressure in the logistic regression analysis may seem debatable since we cannot rule out causality (via the possible scenario that the Asp-allele leads to a high pulse pressure, which subsequently causes angiographically detectable ARAS), we found that the odds ratio on renal artery stenosis rose when pulse pressure was accounted for. In case of causality we would have expected a decrease of the odds ratio in the adjusted model. Moreover, all reports regarding blood pressure or presence of hypertension and the eNOS Glu298Asp polymorphism in Caucasians are negative. ${ }^{24-27}$ Alternatively, (differences in) the total burden of atherosclerosis may account for our findings. Indeed, other investigators previously found the eNOS Glu298Asp polymorphism to be related to the presence of atherosclerotic manifestations such as coronary artery disease $e^{28-30}$ and carotid atherosclerotic plaques, ${ }^{31}$ although negative reports have also been published. ${ }^{27}$ Since atherosclerotic renovascular disease is generally viewed upon as a local manifestation of systemic atherosclerotic disease, at least our data in ARAS+ are in line with these earlier observations. But even the results in ARAS- patients may be compatible with more severe atherosclerosis in these individuals. The absence of atherosclerotic lesions in renal arteries does not at all exclude the presence of atherosclerosis elsewhere in the vascular system and, indeed, $22 \%$ of the ARAS- patients had experienced signs of extrarenal atherosclerosis. Moreover, it is likely that normotensive and hypertensive patients with relatively mild forms of hypertension in a general care practice have lower amounts of (extra-renal) atherosclerosis than patients in an outpatient clinic of a tertiary referral hospital who are suspected of having renal artery stenosis. In this respect, the difference between ARAS- and ARAS+, namely proven renal artery atherosclerosis, may just be a marker of more wide-spread atherosclerosis. Indirectly, this hypothesis is supported by the differences between the groups regarding pulse pressure, which is commonly associated with the severity of atherosclerosis, although it cannot be ignored that ARAS+ had a far worse risk profile. Yet, since the extent of extra-renal atherosclerosis was not investigated in our study, we cannot exclude other explanations. $^{22,23,32}$

The most obvious candidate genes to look at when renal artery disease is the object of study, the ones of the renin-angiotensin system, were evenly distributed among all groups, at least with respect to the polymorphisms in the genes that we studied. Only few studies have focused on the association between ARAS and genetic polymorphisms and all have addressed the ACE ins/del polymorphism. ${ }^{8-11}$ Two groups compared the distribution of the ACE ins/del genotypes between ARAS and normotensive groups and found the $D$ allele to be more prevalent in ARAS ${ }^{9,11}$. The AGT Met235T and the ATR1 A1166C polymorphisms were not found to be associated with ARAS. ${ }^{11}$ When, on the other hand, patients with ARAS were compared to patients with diffuse atherosclerotic disease who underwent angiography on the suspicion of aortic aneurysm no association was found with the ACE genotype. ${ }^{8}$ The only study that 
compared hypertensive patients with renal artery stenosis to hypertensive patients without renal artery stenosis, found an overrepresentation of DD homozygotes in the renal artery stenosis group, ${ }^{10}$ but it must be emphasized that in only $38 \%$ of the patients angiography of the renal arteries was performed on the suspicion of renovascular hypertension. Our study is the first to examine three different polymorphisms of the renin-angiotensin system in hypertensive patients who were all selected according to the same criteria for angiography. With our standardized approach, we did not find an association between these genotypes and ARAS.

\section{Perspectives}

Taking all data together, we hypothesize that the Asp-allelle of the eNOS Glu298Asp polymorphism predisposes to (systemic) atherosclerosis but that the extent of the lesions, among which those in the renal arteries, depends also on additional risk factors. Considering the importance of endothelium-derived nitric oxide in atherosclerosis, our findings fit very well in the theoretical framework of atherosclerotic disease and merit further research into the mechanisms whereby altered nitric oxide production in conjunction with other cardiovascular risk factors may cause systemic and/or renal atherosclerosis. 


\section{References}

1. McLaughlin K, Jardine AG, Moss JG. ABC of arterial and venous disease. Renal artery stenosis. BMJ. 2000;320:1124-7.

2. Agerholm-Larsen B, Nordestgaard BG, Tybjaerg-Hansen A. ACE gene polymorphism in cardiovascular disease: meta-analyses of small and large studies in whites. Arterioscler Thromb Vasc Biol. 2000;20:484-92.

3. Staessen JA, Wang JG, Ginocchio G, Petrov V, Saavedra AP, Soubrier F, Vlietinck R, Fagard $\mathrm{R}$. The deletion/insertion polymorphism of the angiotensin converting enzyme gene and cardiovascular-renal risk. J Hypertens. 1997;15:1579-92.

4. Spiering W, Kroon AA, Fuss-Lejeune MM, Daemen MJ, de Leeuw PW. Angiotensin II sensitivity is associated with the angiotensin II type 1 receptor $A(1166) C$ polymorphism in essential hypertensives on a high sodium diet. Hypertension. 2000;36:411-6.

5. Tiret L, Bonnardeaux A, Poirier O, Ricard S, Marques-Vidal P, Evans A, Arveiler D, Luc G, Kee F, Ducimetiere $P$, Soubrier $F$, Cambien $F$. Synergistic effects of angiotensin-converting enzyme and angiotensin-II type 1 receptor gene polymorphisms on risk of myocardial infarction. Lancet. 1994;344:910-3.

6. Hopkins PN, Hunt SC, Jeunemaitre X, Smith B, Solorio D, Fisher NDL, Hollenberg NK, Williams GH. Angiotensinogen Genotype Affects Renal and Adrenal Responses to Angiotensin II in Essential Hypertension. Circulation. 2002;105:1921-7.

7. Veldman BA, Spiering W, Doevendans PA, Vervoort G, Kroon AA, de Leeuw PW, Smits P. The Glu298Asp polymorphism of the NOS 3 gene as a determinant of the baseline production of nitric oxide. J Hypertens. 2002;20:2023-7.

8. Losito A, Parente B, Cao PG, Jeffery S, Afzal AR. ACE gene polymorphism and survival in atherosclerotic renovascular disease. Am J Kidney Dis. 2000;35:211-5.

9. Missouris CG, Barley J, Jeffery S, Carter ND, Singer DR, MacGregor GA. Genetic risk for renal artery stenosis: association with deletion polymorphism in angiotensin 1-converting enzyme gene. Kidney Int. 1996;49:534-7.

10. Olivieri O, Grazioli S, Pizzolo F, Stranieri C, Trabetti E, Beltrame F, Girelli D, Pignatti PF, Corrocher R. Different impact of deletion polymorphism of gene on the risk of renal and coronary artery disease. J Hypertens. 2002;20:37-43.

11. Olivieri O, Trabetti E, Grazioli S, Stranieri C, Friso S, Girelli D, Russo C, Pignatti PF, Mansueto G, Corrocher R. Genetic polymorphisms of the renin-angiotensin system and atheromatous renal artery stenosis. Hypertension. 1999;34:1097-100.

12. Safian RD, Textor SC. Renal-artery stenosis. N Engl J Med. 2001;344:431-42.

13. Caps MT, Perissinotto C, Zierler RE, Polissar NL, Bergelin RO, Tullis MJ, Cantwell-Gab K, Davidson RC, Strandness DE, Jr. Prospective study of atherosclerotic disease progression in the renal artery. Circulation. 1998;98:2866-72.

14. Henskens LH, Spiering W, Stoffers HE, Soomers FL, Vlietinck RF, de Leeuw PW, Kroon AA. Effects of ACE I/D and AT1R-A1166C polymorphisms on blood pressure in a healthy normotensive primary care population: first results of the Hippocates study. $J$ Hypertens. 2003;21:81-6.

15. Sham P. Statistics in human genetics. London: Arnold; 1998.

16. Confounding and interaction in regression. In: Kleinbaum DG, Kupper LL, Muller KE, eds. Applied regression analysis and other multivariable methods. Second ed. Boston: PWSKENT Publishing Company; 1988:163-80.

17. Berglund G, Andersson O, Wilhelmsen L. Prevalence of primary and secondary hypertension: studies in a random population sample. BMJ. 1976;2:554-6.

18. Sigurdsson JA, Bengtsson C, Tibblin E, Wojciechowski J. Prevalence of secondary hypertension in a population sample of Swedish women. Eur Heart J. 1983;4:424-33.

19. Lewin A, Blaufox MD, Castle H, Entwisle G, Langford H. Apparent prevalence of curable hypertension in the Hypertension Detection and Follow-up Program. Arch Intern Med. $1985 ; 145: 424-7$. 
20. Detection, evaluation, and treatment of renovascular hypertension. Final report. Working Group on Renovascular Hypertension. Arch Intern Med. 1987;147:820-9.

21. Hollis S. A graphical sensitivity analysis for clinical trials with non-ignorable missing binary outcome. Stat Med. 2002;21:3823-34.

22. Cardon LR, Palmer LJ. Population stratification and spurious allelic association. Lancet. 2003;361:598-604.

23. Tiret L, Cambien F. Departure from Hardy-Weinberg equilibrium should be systematically tested in studies of association between genetic markers and disease. Circulation. 1995;92:3364-5.

24. Karvonen J, Kauma H, Kervinen K, Rantala M, Ikaheimo M, Paivansalo M, Savolainen MJ, Kesaniemi YA. Endothelial nitric oxide synthase gene Glu298Asp polymorphism and blood pressure, left ventricular mass and carotid artery atherosclerosis in a population-based cohort. J Intern Med. 2002;251:102-10.

25. Jachymova M, Horky K, Bultas J, Kozich V, Jindra A, Peleska J, Martasek P. Association of the Glu298Asp polymorphism in the endothelial nitric oxide synthase gene with essential hypertension resistant to conventional therapy. Biochem Biophys Res Commun. 2001;284:426-30.

26. Markus HS, Ruigrok Y, Ali N, Powell JF. Endothelial nitric oxide synthase exon 7 polymorphism, ischemic cerebrovascular disease, and carotid atheroma. Stroke. 1998;29:1908-11.

27. Jeerooburkhan N, Jones LC, Bujac S, Cooper JA, Miller GJ, Vallance P, Humphries SE, Hingorani AD. Genetic and environmental determinants of plasma nitrogen oxides and risk of ischemic heart disease. Hypertension. 2001;38:1054-61.

28. Colombo MG, Andreassi MG, Paradossi U, Botto N, Manfredi S, Masetti S, Rossi G, Clerico A, Biagini A. Evidence for association of a common variant of the endothelial nitric oxide synthase gene (Glu298-->Asp polymorphism) to the presence, extent, and severity of coronary artery disease. Heart. 2002;87:525-8.

29. Hingorani AD, Liang CF, Fatibene J, Lyon A, Monteith S, Parsons A, Haydock S, Hopper RV, Stephens NG, O'Shaughnessy KM, Brown MJ. A common variant of the endothelial nitric oxide synthase (Glu298- >Asp) is a major risk factor for coronary artery disease in the UK. Circulation. 1999;100:1515-20.

30. Paolo Rossi G, Cesari M, Zanchetta M, Colonna S, Maiolino G, Pedon L, Cavallin M, Maiolino $\mathrm{P}$, Pessina AC. The T-786C endothelial nitric oxide synthase genotype is a novel risk factor for coronary artery disease in Caucasian patients of the GENICA study. Journal of the American College of Cardiology. 2003;41:930-7.

31. Lembo G, De Luca N, Battagli C, lovino G, Aretini A, Musicco M, Frati G, Pompeo F, Vecchione C, Trimarco B. A common variant of endothelial nitric oxide synthase (Glu298Asp) is an independent risk factor for carotid atherosclerosis. Stroke. 2001;32:735-40.

32. Deng HW, Chen WM, Recker RR. Population admixture: detection by Hardy-Weinberg test and its quantitative effects on linkage-disequilibrium methods for localizing genes underlying complex traits. Genetics. 2001;157:885-97. 


\section{Appendix}

\section{Sensitivity analysis}

For the purpose of genetic analysis, we assumed renal artery stenosis to be absent in the hypertensive subjects from the general care practice. However, in the past, several studies reported a prevalence of secondary hypertension, among which renal artery stenosis, of 1-5 \% in such groups. ${ }^{1-4}$ We tested the robustness of the association between eNOS 298Asp and renal artery stenosis by modeling OR's when we excluded $5 \%$ ( 8 subjects, 16 alleles) of the hypertensive subjects of the general care practice. This was done with all possible allele combinations since deleting 16 Glu allele has a different effect on the OR, than deleting 16 Asp alleles. Since the control group consisted of 201 Glu alleles and 107 Asp allele, modeled allele numbers of the hypertensive controls the sensitivity analysis are thus (with $0 \leq r \leq 16$ ) 201-r Glu alleles and $91+r$ Asp alleles.

Odds ratios were calculated as:

$\frac{\text { no. Glu-alleles (controls) / no. Asp-alleles (controls) }}{\text { no. Glu-alleles (ARAS+) / no. Asp-alleles (ARAS }+ \text { ) }}=\frac{(201-r) /(91+r)}{115 / 103}$

Figure 4.I and Table 4.II show the modeled OR's (95\%-Confidence interval) for all values of $r$. Even in the unlikely event that in reality $5 \%$ of the hypertensive patients from the general practice had renal artery stenosis and would therefore have to be omitted, the relationship we found between the eNOS Glu298Asp polymorphism and renal artery stenosis is still statistically significant. This holds true for the model in which all omitted subjects had the Asp/Asp genotype (OR 1.98 (1.38-2.85)), as well as for the model in which all excluded subjects had the Glu/Glu genotype (OR 1.55 (1.08-2.21)), and also for all intermediate possibilities.

\section{Refrences}

1. Berglund G, Andersson O, Wilhelmsen L. Prevalence of primary and secondary hypertension: studies in a random population sample. Br Med J. 1976;2:554-6.

2. Sigurdsson JA, Bengtsson C, Tibblin E, Wojciechowski J. Prevalence of secondary hypertension in a population sample of Swedish women. Eur Heart J. 1983;4:424-33.

3. Lewin A, Blaufox MD, Castle H, Entwisle G, Langford H. Apparent prevalence of curable hypertension in the Hypertension Detection and Follow-up Program. Arch Intern Med. 1985;145:424-7.

4. Detection, evaluation, and treatment of renovascular hypertension. Final report. Working Group on Renovascular Hypertension. Arch Intern Med. 1987;147:820-9. 
Figure 4.I Sensitivity analysis

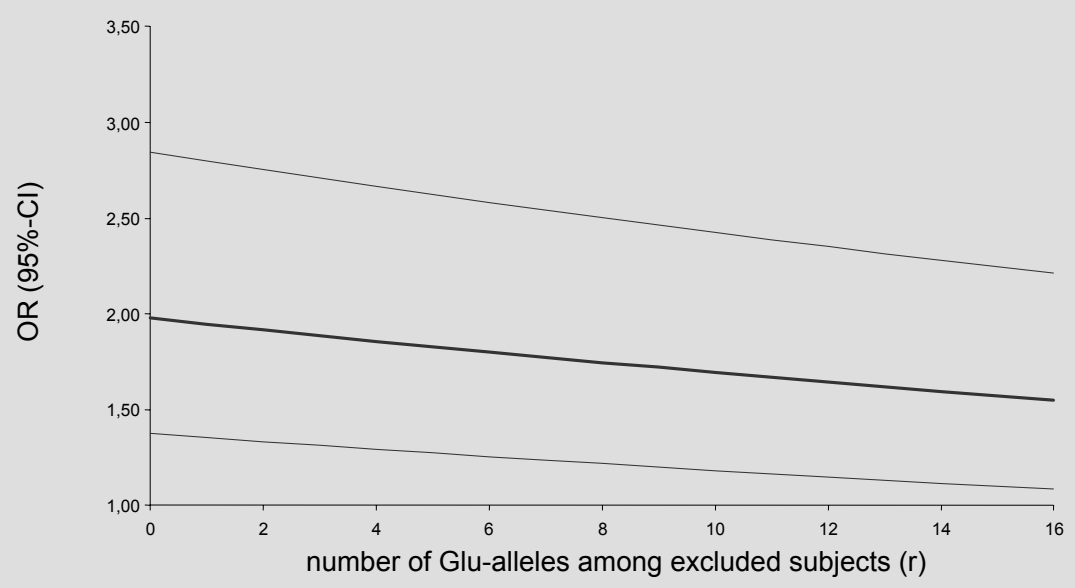

Modelling the effect of $5 \%$ prevalence of renal artery stenosis among the hypertensives from the general practice, who would therefore have to be excluded, on the relationship between renal artery abonormalities and the eNOS Glu298Asp.

Table 4.II Sensitivity analysis.

\begin{tabular}{lccc}
$r^{\mathrm{a}}$ & Glu $^{\mathrm{b}}$ & Asp $^{\mathrm{c}}$ & OR (95\%-CI) \\
\hline 0 & 201 & 91 & $1.98(1.38-2.85)$ \\
1 & 200 & 92 & $1.95(1.36-2.80)$ \\
2 & 199 & 93 & $1.92(1.33-2.75)$ \\
3 & 198 & 94 & $1.89(1.31-2.71)$ \\
4 & 197 & 95 & $1.86(1.26-2.67)$ \\
5 & 196 & 96 & $1.83(1.27-2.62)$ \\
6 & 195 & 97 & $1.80(1.26-2.58)$ \\
7 & 194 & 98 & $1.77(1.24-2.54)$ \\
8 & 193 & 99 & $1.75(1.22-2.50)$ \\
9 & 192 & 100 & $1.72(1.20-2.46)$ \\
10 & 191 & 101 & $1.69(1.18-2.43)$ \\
11 & 190 & 102 & $1.67(1.17-2.39)$ \\
12 & 189 & 103 & $1.64(1.15-2.35)$ \\
13 & 188 & 104 & $1.62(1.13-2.31)$ \\
14 & 187 & 105 & $1.60(1.12-2.28)$ \\
15 & 186 & 106 & $1.57(1.10-2.25)$ \\
16 & 185 & 107 & $1.55(1.08-2.21)$
\end{tabular}

Modelling the effect of $5 \%$ prevalence of renal artery stenosis among the hypertensives from the general practice, who would therefore have to be excluded, on the relationship between renal artery abonormalities and the eNOS Glu298Asp polymorphism ${ }^{a}$ denotes number of deleted Glualleles in the sensitivity analysis; ${ }^{b}$ Number of Glu alleles in the group of hypertensives from the family practice; ${ }^{c}$ Number of Glu alleles in the group of hypertensives from the family practice; ${ }^{d}$ Odds ratio $(95 \%-\mathrm{Cl})$ : hypertensive controls versus ARAS+. 


\section{Chapter 5}

\section{Asymmetry of Renal Blood Flow in Patients with moderate to severe Hypertension}

Marieke van Onna, Alfons J.H.M. Houben, Abraham A. Kroon, Thomas K.A. Wierema, Derk Koster, Jos M.A. van Engelshoven, Peter W. de Leeuw Hypertension 2003;41:108-113 


\section{Abstract}

It is generally assumed that renal blood flow is symmetric in the absence of renal artery stenosis. The aim of the present study was to evaluate whether this really is the case. From a group of consecutive hypertensive patients who had undergone renal angiography, we selected those with patent renal arteries. In all of them selective renal blood flow measurements $\left({ }^{133}\right.$ Xenon washout technique) had been performed with blood sampling from aorta and both renal veins $(n=148)$. Asymmetry of RBF, defined as $\geq 25 \%$ difference in renal blood flow between left and right kidney, was present in $51 \%$ of the patients. Subjects with and without asymmetry did not differ in age, body mass index, blood pressure, creatinine clearance, renal volume or activity of the renin-angiotensin system. The presence of asymmetry coincided with an increased rate of false-positive results on renal scintigraphy. Preliminary data suggest that there may be a relation between asymmetry and renal sympathetic nerve activity. This study demonstrates that asymmetry of renal blood flow is a frequent finding in severe hypertension, which may confound the results of diagnostic tests for renal artery stenosis. Furthermore, the present results underscore the importance of studying the function of both kidneys separately, since it cannot be assumed that they are functionally equal. 


\section{Introduction}

Evaluation of split renal function in patients with elevated blood pressure may be useful as a screening procedure to detect (unilateral) renal artery stenosis. Renal scintigraphy and, more recently, Doppler ultrasonography ${ }^{1}$ are among the tests that have been developed for this purpose. The implicit assumption underlying these diagnostic tests is that, in the absence of a hemodynamically significant renal artery stenosis, blood flow through the kidneys will be roughly symmetrical. Whether this is truly the case, however, has not been proven.

In our clinic, we routinely perform selective renal blood flow (RBF) studies in all patients in whom renal angiography is performed on the suspicion of renovascular hypertension. During these investigations we frequently noted differences in renal blood flow between the left and the right kidney in patients without angiographic abnormalities. Whereas asymmetry of RBF is the hallmark of (unilateral) renal artery stenosis, inequality in RBF between two kidneys in patients with patent renal arteries is a more elusive phenomenon.

To assess how often asymmetry of RBF exists in patients without renal artery stenosis and to search for possible determinants of this asymmetry, we have re-evaluated the blood flow data that were obtained in a large group of patients who had all undergone the same study protocol.

\section{Methods}

\section{Study protocol}

Since February 1994, all patients who are referred to our clinic for evaluation of their hypertension (i.e. diastolic blood pressure above $90 \mathrm{mmHg}$ on at least three occasions) are subjected to a standard diagnostic protocol. This includes renal angiography with selective renal blood flow measurements and arterial and renal venous blood sampling when patients fulfil one or more of the following criteria: hypertension despite two or more antihypertensive drugs, accelerated hypertension, documented atherosclerotic vascular disease in two or more vascular beds, the presence of an abdominal bruit or an unexplained impairment of renal function in response to antihypertensive treatment.

For reasons of standardisation, all antihypertensive medication is discontinued for three weeks and patients are requested to use a sodium-restricted diet $(55 \mathrm{mmol} / 24 \mathrm{~h})$ during the week preceding the study. Compliance with the diet is estimated from a 24 hour collection of urine. After an overnight fast, the aorta and both renal veins are cannulated via the femoral route and blood samples are drawn simultaneously from the aorta and 
renal veins for determination of active plasma renin concentration (APRC) in all patients and angiotensin II, aldosterone and norepinephrine in subgroups as part of other study protocols. Subsequently, we measure selective mean renal blood flow (MRBF) by means of the ${ }^{133}$ Xenon washout technique ${ }^{2,3}$ as described previously. ${ }^{4,5}$ As a matter of routine, we start ${ }^{133}$ Xenon washout studies in the left kidney and the whole Xenon-washout procedure for both kidneys is completed within approximately ten minutes. Blood pressure and heart rate are monitored during the MRBF measurements and no contrast material is administered before the flow studies have been completed. Thereafter, intraarterial renal angiography is performed with a commercially available digital subtraction system (Integris 5000; Philips Medical Systems; Best, The Netherlands). A radiologist, who is unaware of the results from the renal blood flow studies, evaluates the angiographic films.

The ${ }^{133}$ Xenon washout curves are analysed off-line using a two-phase exponential model, after subtraction of background radiation. ${ }^{2,3}$ (GraphPad Software, Inc., San Diego, CA). In our hands, the $95^{\text {th }}$ percentile of differences between two repeated flow measurements in the same kidney is $25 \%$ (mean variability of two measurements plus two times standard deviation), with no systematic left-right differences between the first and the second measurements.

All patients gave written informed consent and the Maastricht University Hospital Medical Ethics Committee approved the study.

\section{Renal scintigraphy}

In the early phase of the study period, renal scintigraphy was still used as a screening test for renal artery stenosis. Renography was always performed with Technetium-99m labelled mercaptoacetyltriglycine $\left({ }^{99 \mathrm{~m}} \mathrm{Tc}-\mathrm{MAG}_{3}\right)$, initially under baseline conditions, later on also after the ingestion of $50 \mathrm{mg}$ of captopril. Since a recent meta-analysis did not demonstrate any superiority of carrying out both a baseline and a postcaptopril study above a postcaptopril study alone, ${ }^{6}$ results from all renographic observations were pooled for analysis.

\section{Kidney volume measurements}

As of January 1999, all patients clinically suspected of having a renal artery stenosis underwent computed tomography angiography (CTA) of the renal arteries on the day prior to intra-arterial angiography. Spiral CT examinations were performed with a CT Twin RTS (Elscint, Haifa, Israel) during inspiratory breath-hold with the administration of $140 \mathrm{ml}$ iohexol (Omnipaque 300; Nycomed, Oslo, Norway) and a scan delay time of 18 seconds. The contrast-enhanced scans were obtained using a $2.7 \mathrm{~mm}$ collimation slice thickness and a pitch of 0.7. A 430-mm field of view and a $512 \times 512$ matrix with a reconstruction interval of $1.3 \mathrm{~mm}$ were used. CT-imaging series were only used to 
calculate kidney volume when both kidneys were depicted completely and the subject had been able to hold his/her breath sufficiently long to allow acceptable imaging. The original images were reconstructed in a coronal plane with a $5 \mathrm{~mm}$ slice thickness without an intersection gap. On each created image the boundaries of both kidneys were traced manually. In cases of partial voluming, i.e. a voxel containing both kidney and surroundings, the segmentation line was drawn halfway. The renal volume was then calculated automatically by adding all voxel volumes lying within the borders of the kidney.

\section{Assay methods}

APRC was measured using a two-side immunoradiometric assay method. ${ }^{7}$ Angiotensin II and aldosterone were determined after extraction from plasma by radio-immunoassay using highly specific antibodies. ${ }^{8,9}$ A sensitive HPLC method with fluorimetric detection was used to determine norepinephrine concentrations. ${ }^{10}$

\section{Calculations and statistics}

Creatinine clearance was estimated from the Cockcroft formula. ${ }^{11}$ MRBF ratio was calculated by dividing flow in the kidney with the highest MRBF by that in the kidney with the lowest MRBF. In absence of asymmetry in MRBF, we expected a maximum difference between left and right kidney MRBF of approximately 25\% (mean variability of two measurements in the same kidney plus two times the standard deviation). We therefore defined symmetry of MRBF as $<25 \%$ difference between left and right MRBF and asymmetry of MRBF as $\geq 25 \%$ difference. According to this definition we divided study participants into two groups. Differences between the symmetry and the asymmetry group in patient characteristics and MRBF were analysed using unpaired t-tests and the Pearson Chi-Square test. Friedman's test was applied for analysis of repeated measurements of blood pressure and heart rate. Since the distributions of MRBF ratio, kidney volume and neurohormones were not normal, nonparametric tests, including the Mann-Whitney $U$ test, the Wilcoxon signed rank test and the Spearman analysis for correlations, were applied to test differences in these variables. The nonparametric Chi-Square test was used to assess differences in the rate of false-positive results on scintigraphy. For statistical analyses, SPSS 10.0 was used. A p-value of $<0.05$ was considered statistically significant. Data with a normal distribution are presented as means $\pm S D$, otherwise medians and interquartile ranges are given. 


\section{Results}

Between February 1994 and February 2001, combined angiography and selective renal blood flow studies were successfully performed in 233 patients who were without treatment at that time. Of these, 85 patients were excluded from analysis. Reasons for exclusion are listed in Table 5.1. The study group consisted of 90 males and 58 females with a mean age of $51 \pm 13$ years. None had angiographic or biochemical abnormalities suggestive of secondary hypertension. Prior to study, patients were taking medication of $2 \pm 1$ (range $0-5$ ) classes of antihypertensive drugs. The withdrawal of treatment was tolerated well in the majority of patients, no patients were endangered. Systolic blood pressure at admission averaged $175 \pm 28 \mathrm{mmHg}$ and the diastolic blood pressure $105 \pm 15 \mathrm{mmHg}$. One patient had a blood pressure $<135 / 85$, but on other occasions he had blood pressures $>140 / 90$. The average blood pressure during ${ }^{133}$ Xenon washout $(178 \pm 30 / 101 \pm 17)$ was similar to the average blood pressure at admission, and, also, during our measurements, mean arterial pressure and heart rate did not change (data not shown). The average amount of sodium in a 24 hour urine collection was 82 (53-125) $\mathrm{mmol}$. Average creatinine clearance was $93 \pm 34 \mathrm{ml} / \mathrm{min}$; in 23 patients $(16 \%)$ creatinine clearance was lower than $60 \mathrm{ml} / \mathrm{min}$.

Table 5.1. Reasons for exclusion of patients from the analyses.

Reasons for exclusion

Renal artery abnormalities

Atrophic kidney

History of percutaneous transluminal renal angioplasty (PTRA)

Vasovagal collapse during measurements

Inaccurate positioning of catheter

Other

Total

\section{Number of persons}

71

3

3

2

5

85

\section{Renal blood flow}

Mean values for left and right MRBF in the entire group were not different $(183 \pm 68$ vs. $193 \pm 78 \mathrm{ml} / 100 \mathrm{~g} / \mathrm{min}, \mathrm{NS})$. Asymmetry of MRBF was found in 75 patients (51\%). Clinical characteristics of the symmetry and asymmetry groups are shown in Table 5.2. Groups did not differ with regard to age, gender, body mass index, blood pressure, heart rate and renal function.

In patients with an asymmetric MRBF, median ratio was 1.52 (1.34-1.88) and in eleven $(15 \%)$ cases MRBF ratio exceeded 2.00 (Figure 5.1). In subjects with symmetric MRBF, median flow ratio was 1.09 (1.05-1.15). In patients with asymmetric perfusion, left MRBF was on average lower than the right MRBF $(163 \pm 68$ vs. $187 \pm 90 \mathrm{ml} / 100 \mathrm{~g} / \mathrm{min}, p=0.008)$ 
When patients with asymmetric flow were compared to those with symmetric flow, left MRBF was again lower in the former $(163 \pm 68$ vs. $204 \pm 62 \mathrm{ml} / 100 \mathrm{~g} / \mathrm{min}, \mathrm{p}=0.001)$, while right MRBF was similar ( $187 \pm 90$ vs. $200 \pm 64 \mathrm{ml} / 100 \mathrm{~g} / \mathrm{min}$, NS). Although this suggests that asymmetry is predominantly caused by a reduction of left MRBF, right MRBF was lower than left MRBF in 31 out of the 75 subjects (41\%) with an asymmetric flow pattern. The MRBF ratio, or in other words the degree of asymmetry, was similar in those with dominance of left and those with dominance of right MRBF (1.57 (1.35-1.87) vs. 1.39 (1.31-1.88), NS). The percentage distribution of renal blood flow to the cortex (fast component of the ${ }^{133}$ Xe-washout curve) was similar between the symmetry and the asymmetry group. When patients with reduced kidney function (creatinine clearance equal or lower than $60 \mathrm{ml} / \mathrm{min}$ ) were left out of the analysis, similar findings were obtained with respect to asymmetry and left-right differences.

Table 5.2 Clinical characteristics of hypertensive subjects with symmetric and asymmetric renal blood flow.

\begin{tabular}{lccc} 
Clinical characteristics & Symmetry & Asymmetry & $\mathrm{p}$ \\
\hline Age (year) & $50 \pm 13$ & $51 \pm 13$ & $\mathrm{NS}$ \\
Male & $45(62)$ & $45(60)$ & $\mathrm{NS}$ \\
$\mathrm{BMI}\left(\mathrm{kg} / \mathrm{m}^{2}\right.$ ) & $28 \pm 5$ & $28 \pm 5$ & $\mathrm{NS}$ \\
No. of classes of antihypertensive drugs prior to study & $2 \pm 1$ & $2 \pm 1$ & $\mathrm{NS}$ \\
Calcium-antagonists & $44(60)$ & $32(43)$ & $\mathrm{NS}$ \\
$\beta$-blockers & $41(56)$ & $39(52)$ & $\mathrm{NS}$ \\
Diuretics & $27(37)$ & $19(25)$ & $\mathrm{NS}$ \\
Angiotensin-Converting Enzyme inhibitor & $25(34)$ & $25(33)$ & $\mathrm{NS}$ \\
Angiotensin Type 1 receptor antagonists & $14(19)$ & $14(19)$ & $\mathrm{NS}$ \\
Other (a-blockers, centrally-acting agents, direct vasodilators) & $9(12)$ & $11(15)$ & $\mathrm{NS}$ \\
$\mathrm{SBP}^{\mathrm{a}}$ (mmHg) & $180 \pm 31$ & $177 \pm 29$ & $\mathrm{NS}$ \\
$\mathrm{DBP}^{\mathrm{a}}$ (mmHg) & $101 \pm 17$ & $100 \pm 17$ & $\mathrm{NS}$ \\
$\mathrm{HR}^{\mathrm{a}}$ (beats per minute) & $71 \pm 15$ & $73 \pm 14$ & $\mathrm{NS}$ \\
Urinary sodium (mmol/24 hour) $_{\text {Creatinine clearance (ml/min) }}$ & $83(52-121)$ & $79(56-127)$ & $\mathrm{NS}$ \\
No. of persons with $>2$ renal arteries & $94 \pm 36$ & $91 \pm 32$ & $\mathrm{NS}$ \\
& 23 & 24 & $\mathrm{NS}$
\end{tabular}

Values are presented as means \pm SD or median (interquartile range) number (\% of group); BMI denotes body mass index, No. number, SBP systolic blood pressure, DBP diastolic blood pressure and HR heart rate. ${ }^{a}$ measured during ${ }^{133}$ Xenon washout.

\section{Renal scintigraphy}

Renal scintigraphy had been performed in twelve subjects from the symmetry group and sixteen patients of the asymmetry group. In seven of the patients with asymmetry of MRBF (44\%), renal scintigraphy suggested renal artery stenosis, whereas this was the case in only one of the subjects with symmetry of MRBF (9\%). This difference was statistically significant $(p=0.02)$. As judged by renal angiography, all of these subjects had patent renal arteries indicating that renal scintigraphy results that suggested renal artery stenosis must be false positive. Creatinine clearance was comparable between subjects with positive and negative results on scintigraphy. 
Figure 5.1 Frequency distribution of mean renal blood flow (MRBF) ratio in 148 patients with essential hypertension.

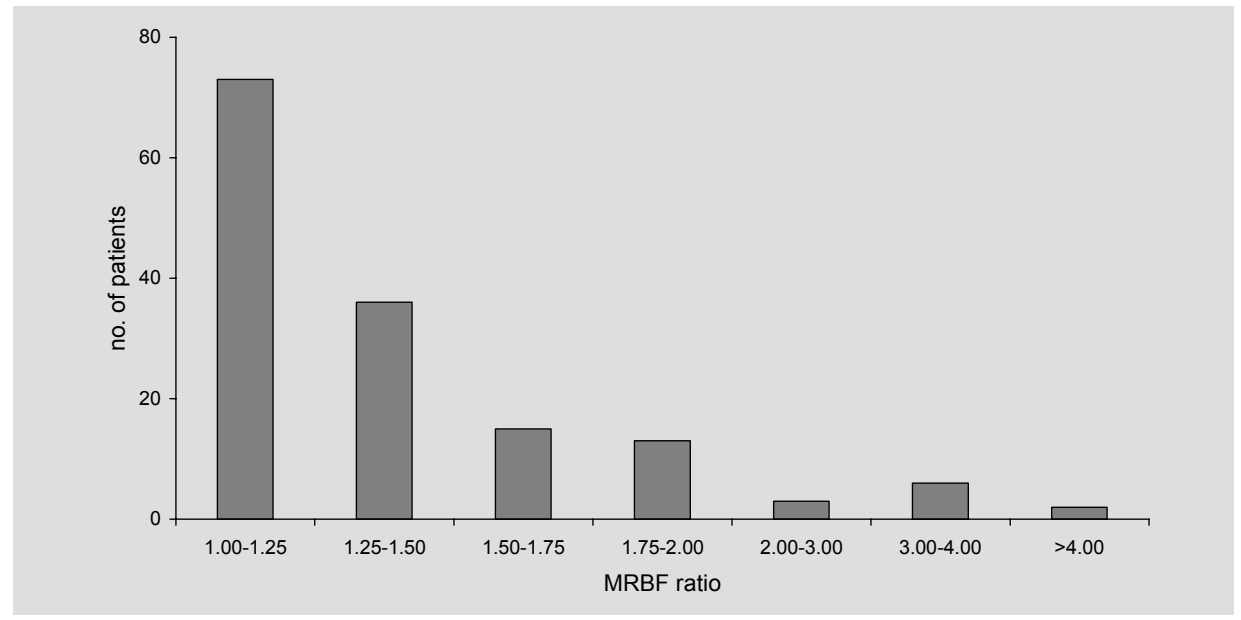

\section{Kidney volume}

Adequate kidney volume measurements could be obtained in fourteen patients with symmetric renal blood flow and nine patients with asymmetric perfusion. Renal volume did not differ between left and right kidneys, either in the symmetry group (187 $\mathrm{ml}$ (158$205)$ vs. $185 \mathrm{ml}$ (166-203), NS) or in the asymmetry group (154 ml (122-179) vs. $151 \mathrm{ml}$ (133-217), NS). When all volumes of the kidneys with the lowest flow were compared with those of kidneys with the highest flow, regardless of side, no differences were found either. This was true for patients with symmetry (184 ml (166-214) vs. 187 (153$196)$, NS) as well as for those with asymmetry of MRBF (151 (128-217) vs. 154 (130$178)$, NS). Although kidney volumes tended to be a bit lower in the asymmetry group as compared to the symmetry group, differences were not statistically significant. MRBF, which is expressed per $100 \mathrm{~g}$ kidney mass, and kidney volume did not correlate (Figure 5.2 ).

\section{Neurohormones}

Arterial and selective renal venous concentrations of renin, angiotensin II and aldosterone (Table 5.3) and veno-arterial differences of renin and angiotensin II did not differ between the two groups. In the asymmetry group, no differences in these hormones were found between the kidneys with the highest and the lowest MRBF. Since the left adrenal vein drains into the left renal vein, we used norepinephrine values from the left renal vein only when mixing with blood from the adrenal gland could virtually be excluded on the basis of concurrent aldosterone measurements. In each patient the difference between the arterial and the venous concentration of 
norepinephrine was calculated for the kidney with the lowest flow. The same was done for the kidney with the highest MRBF. Although numbers were low, there tended to be a relation between the veno-arterial differences of norepinephrine and MRBF ratio, in patients with a ratio up to 2.00. This was of borderline significance when the norepinephrine gradient across the kidneys with the lowest MRBF was taken as independent variable $(r=0.61, p=0.06)$ (Figure 5.3).

Figure 5.2 Scatterplot of mean renal blood flow (MRBF) (Y-axis) and kidney volume (X-axis).

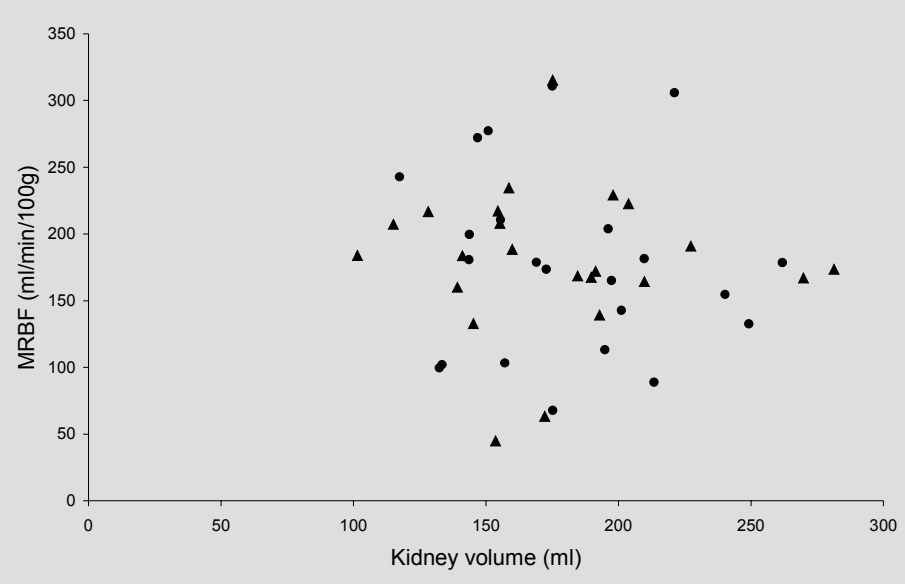

There is no correlation between left MRBF and left kidney volume (Spearman's rho, $r=-0.03$, NS) nor between right MRBF and right kidney volume (Spearman's rho, $r=-0.14, N S)$; $\Delta$ left kidney,

- right kidney.

Table 5.3 Arterial and selective renal vein concentrations of renin, angiotensin II and aldosterone in hypertensive subjects with symmetric and asymmetric renal blood flow.

\begin{tabular}{lllll} 
& & \multicolumn{1}{c}{ Symmetry } & \multicolumn{1}{c}{ Asymmetry } & $\mathrm{p}$ \\
\hline Renin $(\mu \mathrm{U} / \mathrm{ml})$ & Arterial & $17.9(11.8-28.4)$ & $20.9(11.2-38.9)$ & $\mathrm{NS}$ \\
$(\mathrm{n}=72 \mathrm{vs} . \mathrm{n}=75)$ & Right renal vein & $22.1(14.2-34.6)$ & $23.8(12.8-46.1)$ & $\mathrm{NS}$ \\
& Left renal vein & $21.7(14.1-36.5)$ & $24.1(12.8-40.0)$ & $\mathrm{NS}$ \\
Angiotensin II $(\mathrm{pg} / \mathrm{ml})$ & Arterial & $11.4(8.0-15.7)$ & $12.9(8.7-15.5)$ & $\mathrm{NS}$ \\
$(\mathrm{n}=22$ vs. $\mathrm{n}=21)$ & Right renal vein & $11.2(8.6-15.1)$ & $11.6(8.7-13.0)$ & $\mathrm{NS}$ \\
& Left renal vein & $12.5(10.1-18.7)$ & $11.4(9.9-14.9)$ & $\mathrm{NS}$ \\
Aldosterone $(\mathrm{pg} / \mathrm{ml})$ & Arterial & $0.38(0.20-0.73)$ & $0.26(0.17-0.51)$ & $\mathrm{NS}$ \\
$(\mathrm{n}=14$ vs. $\mathrm{n}=21)$ & Right renal vein & $0.36(0.23-0.65)$ & $0.22(0.14-0.44)$ & $\mathrm{NS}$ \\
& Left renal vein & $0.38(0.19-0.60)$ & $0.22(0.16-0.27)$ & $\mathrm{NS}$
\end{tabular}

Values are presented as median (interquartile range) 
Figure 5.3 Scatterplot of mean renal blood flow (MRBF) ratio and the veno-arterial differences for norepinephrine in kidneys with the lowest MRBF.

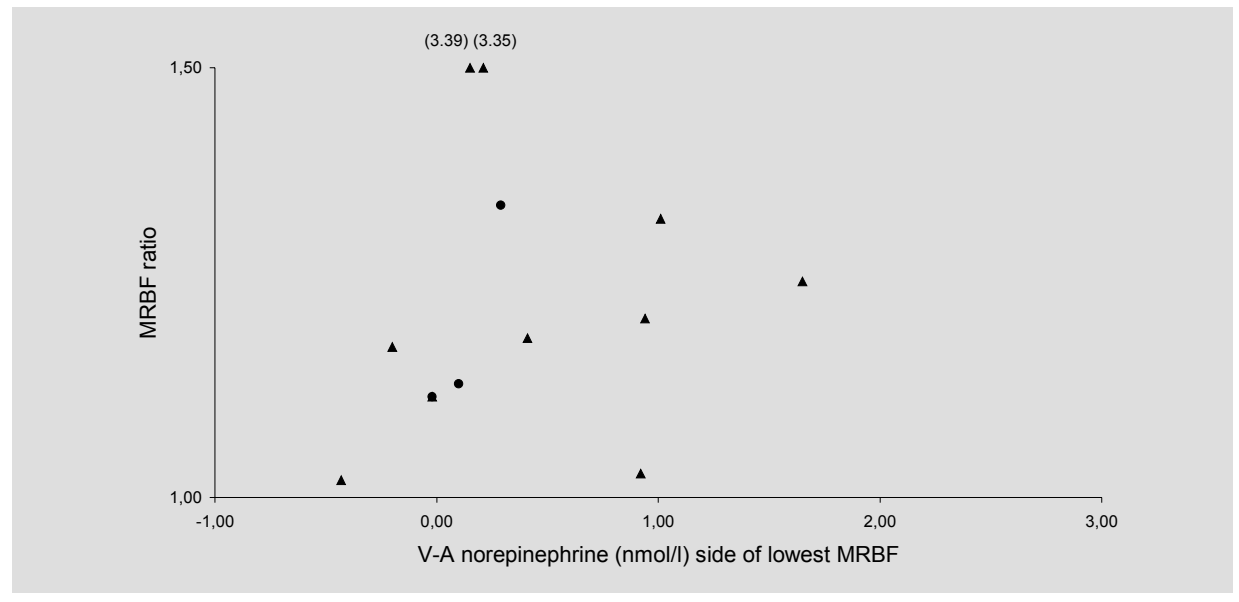

The correlation between the MRBF ratio and the veno-arterial difference for norepinephrine in the kidney with the lowest MRBF was borderline significant in subjects with MRBF ratio $<2.00$ (Spearman's rho, $r=0.61, p=0.06$ ). $\bullet$ right kidney $\boldsymbol{\Delta}$ left kidney Numbers in brackets correspond to the actual ratio of outlying values.

\section{Discussion}

This study shows that substantial differences between left and right renal blood flow may be present in up to $51 \%$ of hypertensive patients with angiographically proven patent renal arteries. Inequality in perfusion between kidneys has been observed in only a few studies. Kioschos et al. determined selective renal blood flow by means of the dye dilution technique in five normotensive controls and fifteen essential hypertensives, including eight subjects who were suspected of having nephrosclerosis. ${ }^{12}$ In six of the 15 essential hypertensives differences in flow between kidneys exceeded $25 \%$, while asymmetry was not found in the normotensives. In a study performed by Baldwin et al. selective clearances of inulin and $p$-aminohippurate were used to investigate subjects who were judged to be in the early stage of essential hypertension. ${ }^{13}$ They defined disparity of effective renal plasma flow (ERPF) as being abnormal when it exceeded the $90^{\text {th }}$ percentile of differences between two kidneys in subjects with normotension and found this to be present in 19 out of 36 patients. Interestingly, in 16 of these patients this was accompanied by a concordant disparity of the glomerular filtration rate (GFR). In a more recent study, Van Jaarsveld et al. evaluated in a large group of hypertensive patients Technetium-99 labeled diethylenetriamine pentaacetic acid ( ${ }^{99 m}$ Tc-DTPA) scintigraphy. ${ }^{14}$ They noticed that, without captopril challenge, the single kidney fractional uptake was statistically significantly lower on the left side than on the right side $(46 \%$ vs. 
54\%). Unfortunately, dye dilution and clearance techniques generate absolute flow values per kidney, whereas scintigraphy results are presented as a percentage contribution of each kidney. So, differences in kidney volume may have confounded these data. On the other hand, the ${ }^{133}$ Xenon-washout technique, which we applied in the present study, measures flow per unit of kidney mass. Therefore, our data on differences in flow between the left and right kidney cannot be explained by differences in kidney mass. To strengthen this point we have determined kidney volumes in a subset of our patients who underwent CT angiography and these data do not point to any association between kidney volume and asymmetry of renal blood flow in our study population either.

From sequential renal blood flow measurements in the same kidney in patients with essential hypertension, Hollenberg et al. ${ }^{15}$ observed differences in flow which were comparable to those between the two kidneys as found by Baldwin et al. ${ }^{13}$ Compared to normotensive controls, renal blood flow in patients with essential hypertension exhibited a 2.2 fold greater variability, which the authors contributed to sinusoid-like oscillations in renal blood flow with a similar cycle length (about 40 seconds) but increased maximal amplitude. ${ }^{15,16}$ It can be concluded from their experiments that the total variability of blood flow in each kidney will randomly result in significant flow differences between both kidneys. Although this phenomenon may have contributed to our results, in our opinion it is not sufficient to explain our observations. After all, we measured ${ }^{133}$ Xenon washout for three minutes, which is almost five times the duration of the oscillatory cycle length, thereby outweighing the effect of oscillations by far.

Inequality of renal blood flow between the kidneys, therefore, is likely to originate from structural or functional differences, or both. The degree of structural abnormalities at the level of interlobar and arcuate arteries has been shown to correlate with renal blood flow and renal vein renin levels. ${ }^{17,18}$ Regarding the symmetry of peripheral abnormalities on renal angiography, literature is inconclusive. Arlart et al. ${ }^{18}$ found approximately symmetric peripheral involvement, whereas Caralps et al. demonstrated asymmetric alterations of the interlobar and arcuate arteries in eleven out of 25 patients. ${ }^{19}$ Interestingly, the latter investigators reported in ten out of the eleven asymmetry cases that the left kidney was more affected than the right one. The smallest renal vessels have been studied in two large histopathologic studies using bilateral kidney biopsies of hypertensive subjects. The severity of arteriosclerotic changes differed between the kidneys in 25 to $39 \%$ of all cases. ${ }^{20,21}$ However, these numbers are difficult to interpret in the light of inter- and intraobserver disagreement rates of $13 \%$ and $20 \%$ respectively. ${ }^{21}$ Additionally, it has been observed that there is also intrarenal heterogeneity of arteriosclerotic changes in the renal cortex. ${ }^{21,22}$ Apart from structural abnormalities, asymmetry in renal perfusion could also be caused by functional differences between kidneys. However, in our population, renin levels did not differ between subjects with symmetry and those with asymmetry. Although not identical to 
norepinephrine release rates $^{23}$ and available only in a small subgroup, veno-arterial differences of norepinephrine were not symmetric in our study and tended to be the highest in patients with more pronounced grades of asymmetry. In patients with differences in MRBF exceeding $200 \%$ this relation is absent, which may indicate that with severe asymmetry different pathophysiological processes are involved. Interestingly, recent data suggest that in isolated renal arteries from WKY rats the vasoconstrictor responses to electric field stimulation are enhanced on the left side, which may be explained by a more dense sympathetic nerve innervation of the left kidney. ${ }^{24}$

Whatever the nature of the (patho)physiologic process(es) leading to asymmetry of renal blood flow, our study shows that the asymmetric phenotype coincides, at least in the subgroup that we were able to study, with an increased rate of false-positive results on renal scintigraphy. This provides evidence that the presence or absence of asymmetry is a consistent characteristic, which may interfere with the accuracy of diagnostic tests that depend on renal blood flow.

An obvious limitation of our study is that we measured MRBF only once in every kidney on one occasion. Therefore, we do not know whether asymmetry is really a consistent trait in a particular person. Secondly, our data were obtained in a selected population of patients who were suspected of renal vascular disease. To what extent our data can be extrapolated to the entire hypertensive population remains elusive. Thirdly, we perform blood flow studies in a fixed order, first left then right. One may argue that our findings are biased by an order effect, especially because we found asymmetry to occur more often at the expense of the left kidney. However, in 31 of the 75 subjects $(41 \%)$ with an asymmetric flow pattern, right RBF was lower than left RBF. In case of a strong and predominant order effect, we would have expected this fraction to be much less. A reason for an order effect may be that the stress related to the measurements would lower the first measurement. However, the fact that heart rate and blood pressure remained constant during the measurements, indicates that the level of arousal did not change during the wash-out studies. Moreover, we did not detect systematic differences between repeated measurements in the same kidney in reproducibility studies. In this context we also like to stress that all measurements are completed within ten minutes, thereby reducing time-dependent variation in renal blood flow as found by Persson et al. in conscious dogs. ${ }^{25}$ Finally, our data leave unanswered the question whether asymmetry is an inborn phenomenon or a consequence of (longstanding) hypertension.

\section{Perspectives}

Over 40 years ago, the first reports regarding asymmetric renal perfusion were published. However, the small study sizes and methodology hampered implement of their findings. We have revisited this subject, and have demonstrated considerable left- 
to-right differences in renal blood flow in a substantial number of subjects in a welldefined group of patients with severe hypertension. Clearly, more studies are necessary to define underlying mechanism(s) and the exact clinical importance of this characteristic. For now, we think our results underscore the importance of studying the function of both kidneys separately, since it cannot be assumed a priori that they are functionally equal. 


\section{References}

1. Radermacher J, Chavan A, Bleck J, Vitzthum A, Stoess B, Gebel MJ, Galanski M, Koch KM, Haller $\mathrm{H}$. Use of Doppler ultrasonography to predict the outcome of therapy for renal-artery stenosis. N Engl J Med. 2001;344:410-7.

2. Hollenberg NK, Mangel R, Fung HY. Assessment of intrarenal perfusion with radioxenon: a critical review of analytical factors and their implications in man. Semin NuCl Med. 1976;6:193-216.

3. Blaufox MD, Fromowitz A, Gruskin A, Meng CH, Elkin M. Validation of use of xenon 133 to measure intrarenal distribution of blood flow. Am J Physiol. 1970;219:440-4.

4. de Leeuw PW, Birkenhager WH. Use of the xenon-133 washout technique in diagnosing renovascular disease in hypertension. Nephron. 1986;44:5-7.

5. Wierema TK, Houben AJ, Kroon AA, Koster D, van der Zander K, van Engelshoven JM, de Leeuw PW. Nitric oxide dependence of renal blood flow in patients with renal artery stenosis. J Am Soc Nephrol. 2001;12:1836-43.

6. Vasbinder GBC, Nelemans PJ, Kessels AG, Kroon AA, de Leeuw PW, van Engelshoven JM. Diagnostic tests for renal artery stenosis in patients suspected of having renovascular hypertension: a meta-analysis. Ann Intern Med. 2001;135:401-11.

7. Simon D, Hartmann DJ, Badouaille G, Caillot G, Guyenne TT, Corvol P, Pau B, Marchand J. Two-site direct immunoassay specific for active renin. Clin Chem. 1992;38:1959-62.

8. Nussberger J, Waeber G, Waeber B, Bidiville J, Brunner HR. Plasma angiotensin-(1-8) octapeptide measurement to assess acute angiotensin-converting enzyme inhibition with captopril administered parenterally to normal subjects. J Cardiovasc Pharmacol. 1988;11: 716-21.

9. Tan SY, Noth R, Mulrow PJ. Direct non-chromatographic radioimmunoassay of aldosterone: validation of a commercially available kit and observations on age-related changes in concentrations in plasma. Clin Chem. 1978;24:1531-3.

10. van der Hoorn FA, Boomsma F, Man in 't Veld AJ, Schalekamp MA. Determination of catecholamines in human plasma by high-performance liquid chromatography: comparison between a new method with fluorescence detection and an established method with electrochemical detection. J Chromatogr. 1989;487:17-28.

11. Cockcroft DW, Gault MH. Prediction of creatinine clearance from serum creatinine. Nephron. 1976;16:31-41.

12. Kioschos JM, Kirkendall WM, Valenca MR, Fitz AE. Unilateral renal hemodynamics and characteristics of dye-dilution curves in patients with essential hypertension and renal disease. Circulation. 1967;35:229-49.

13. Baldwin DS HW, Biggs AW, Gombos EA, Chasis H. Renal function in the separate kidneys of man. II Hemodynamics and excretion of solute and water in essential hypertension. $J$ Clin Invest. 1960;39:395-404.

14. van Jaarsveld BC, Krijnen P, Derkx FH, Oei HY, Postma CT, Schalekamp MA. The place of renal scintigraphy in the diagnosis of renal artery stenosis. Fifteen years of clinical experience. Arch Intern Med. 1997;157:1226-34.

15. Hollenberg NK, Adams DF. The renal circulation in hypertensive disease. Am J Med. 1976;60:773-84.

16. Hollenberg NK, Sandor T. Vasomotion of renal blood flow in essential hypertension. Oscillations in xenon transit. Hypertension. 1984;6:579-85.

17. Hollenberg NK, Epstein M, Basch RI, Merrill JP. "No man's land" of the renal vasculature. An arteriographic and hemodynamic assessment of the interlobar and arcuate arteries in essential and accelerated hypertension. Am J Med. 1969;47:845-54.

18. Arlart IP, Rosenthal J. Peripheral renal vascular disease in essential hypertension: hemodynamic, angiographic, and endocrine assessment. Cardiovasc Intervent Radiol. 1984; 7:221-8.

19. Caralps A, Rius JM, Vilardell M, Magrina N, Sarrias J, Brulles A. Asymmetrical interlobar nephrosclerosis. Lancet. 1974;1:534-536. 
20. Castleman BC SR. The relation of vascular disease to the hypertensive state. II Adequacy of teh renal biopsy as determined from a study of 500 patients. N Engl J Med. 1948;239:729-32.

21. Sommers SC RA, Smithwick RH. Histologic studies of kidney biopsy specimens from patients with hypertension. Am J Pathol. 1958;34:685-715.

22. Kojima S, Yoshitomi Y, Yano M, Saotome M, Tanaka K, Endo M, Kuramochi M. Heterogeneity of renal cortical circulation in hypertension assessed by dynamic computed tomography. Am J Hypertens. 2000;13:346-52.

23. Esler M, Jackman G, Bobik A, Leonard P, Kelleher D, Skews H, Jennings G, Korner P. Norepinephrine kinetics in essential hypertension. Defective neuronal uptake of norepinephrine in some patients. Hypertension. 1981;3:149-56.

24. Sanders MW, Blanco CE, De Mey JGR. Left-right asymmetry of autonomic nervous response in renal (resistance) arteries of rats [Abstract]. Hypertension. 2001;38:983.

25. Persson PB, Ehmke H, Kirchheim HR, Janssen B, Baumann JE, Just A, Nafz B. Autoregulation and non-homeostatic behaviour of renal blood flow in conscious dogs. $J$ Physiol. 1993;462:261-73. 



\section{Chapter 6}

Functional consequences of asymmetry in renal blood flow in hypertensive patients with unilateral atherosclerotic renal artery stenosis

Marieke van Onna, Thomas K.A. Wierema, Alfons J.H.M. Houben, Abraham A. Kroon, Derk Koster, Michiel W. de Haan, Peter W. de Leeuw 


\section{Abstract}

Marked asymmetry of renal blood flow and renin activation are the corner stones of pathophysiology in experimental unilateral renovascular hypertension. In humans, the interrelationships between atherosclerotic unilateral renal artery stenosis (ARAS), renal blood flow and renin actitivity remain to be elucidated, especially since renal patency restoration is not always beneficial. Mean renal blood flow (MRBF; ${ }^{133}$ Xenon washout technique), renin activation and creatinine extraction of both kidneys were determined in hypertensive patients with $(n=59)$ and without $(n=261)$ unilateral ARAS. High(er) grade(s) (of) ARAS $(>50 \%)$ coincided with an increased volume-normalized difference ("asymmetry") in MRBF between the stenosed and the contralateral kidneys. Still, in half of the patients, flow through a kidney with ARAS was similar or even higher. Patients with an asymmetric pattern had a higher renin activity (both high grade and low grade ARAS) and lower extraction of creatinine (only in high grade ARAS) in a kidney with ARAS. Up to one year after revascularisation, patients with a symmetric perfusion had a lower pulse pressure than patients with an asymmetric perfusion, whereas mean arterial pressure was similar. Asymmetric perfusion, rather than the stenosis per se, may be associated with adverse functional consequences. 


\section{Introduction}

In experimental models, renal artery stenosis does not decrease blood flow through the affected kidney until the lumen is reduced by about $75 \% .{ }^{1,2}$ The fall in blood flow is associated with increased renin secretion, which, via angiotensin II mediated efferent vasoconstriction, acts to preserve glomerular filtration rate in the affected kidney. Blood flow through the contralateral kidney rises which leads to a marked perfusion asymmetry between the kidneys. ${ }^{3}$ However, in patients, in whom renal artery stenosis develops more gradually over time, the picture is more complicated. First of all, subtle changes in renal blood flow may already become apparent when the lumen is reduced by as little as $30 \% .{ }^{4}$ Also, the amount of renin activation does not discriminate well between patients with and without renal artery stenosis. ${ }^{5}$ Finally, as we showed previously, also about half of the patients with essential hypertension and patent renal arteries have a significant difference in blood flow through the left and the right kidney. ${ }^{6}$ Thus, the relationship between the degree of luminal reduction of the renal artery, renal blood flow and individual kidney function may be less straightforward than is generally thought. This prompted us to determine the prevalence of asymmetric renal perfusion in patients with unilateral renal artery stenosis, and to compare renin activation and individual kidney function in those with and without asymmetric perfusion. Further, the effect of restoration of renal patency on the blood pressure was compared between these two groups.

\section{Materials and methods}

\section{Study protocol}

Since February 1994, all patients who are referred to our clinic for evaluation of their hypertension (i.e. systolic blood pressure above $140 \mathrm{mmHg}$ and/or diastolic blood pressure above $90 \mathrm{mmHg}$ on at least three occasions) are subjected to a standard diagnostic protocol. This includes renal angiography with selective renal blood flow measurements and arterial and renal venous blood sampling when patients meet one or more of the following criteria: hypertension despite two or more antihypertensive drugs, accelerated hypertension, documented atherosclerotic vascular disease in two or more vascular beds, presence of an abdominal bruit or an unexplained impairment of renal function in response to antihypertensive treatment.

For reasons of standardisation, all antihypertensive medication is discontinued for three weeks and patients are requested to use a sodium-restricted diet $(55 \mathrm{mmol} / 24 \mathrm{~h})$ during the week preceding the study. After an overnight fast, the aorta and both renal veins are cannulated via the femoral route and blood samples are drawn simultaneously from the aorta and renal veins for measurement of active plasma renin concentration (APRC) and creatinine. Subsequently, we measure selective mean renal blood flow (MRBF) by means of the ${ }^{133}$ Xenon washout technique ${ }^{7,8}$ as described previously. ${ }^{9,10}$ We always 
commence ${ }^{133}$ Xenon washout studies in the left kidney and the whole Xenon wash-out procedure for both kidneys is completed within approximately ten minutes. Blood pressure and heart rate are monitored during the MRBF measurements and no contrast agent is administered before the flow studies have been completed. Thereafter, intraarterial renal angiography is performed with a commercially available digital subtraction system (Integris 5000; Philips Medical Systems; Best, The Netherlands).

The ${ }^{133}$ Xenon washout curves are analysed off-line using a two-phase exponential model, after subtraction of background radiation. ${ }^{7,8}$ (GraphPad Software, Inc., San Diego, CA). The first (fast) component is thought to represent cortical renal blood flow, whereas the other component probably cannot be attributed to specific parts. ${ }^{8,9}$ In our hands, the $95^{\text {th }}$ percentile of differences between two repeated flow measurements in the same kidney is $25 \%$ (mean variability of two measurements plus two times the standard deviation), without systematic left-right differences between first and second measurements.

\section{Angiographic films}

Two experienced readers evaluated all angiographic films. In case of abnormalities, they reported on the site and nature of the aberration on standardized forms. In the case of atherosclerotic disease, maximum stenosis was measured as:

(1-(narrowest diameter/diameter of the normal distal main renal artery)) $\times 100 \%$.

If the two estimates of the degree of stenosis differed by more than $10 \%$ (of the highest assessment), a third opinion was decisive.

\section{Clinical follow-up}

Of all patients who underwent angioplasty and/or stenting or surgical renal artery reconstruction, blood pressure values, number of antihypertensive drugs and serum creatinine concentrations were derived from their medical records (three months and one year after the intervention). If patients were not treated at our clinic in that period, we contacted their current physicians. Benefit of revascularisation was defined as a decrease of systolic and/or diastolic blood pressure by at least $15 \mathrm{mmHg}$ while using the same or less antihypertensive drugs. All other outcomes were classified as failure.

All patients gave written informed consent and the Maastricht University Hospital Medical Ethics Committee approved the study.

\section{Assay methods}

Active plasma renin concentration (APRC) was measured using a two-site immunoradiometric assay method. ${ }^{11}$ Standard methods were used for the measurement of creatinine. 


\section{Calculations and definitions}

Creatinine clearance was estimated by the Cockcroft formula. ${ }^{12}$ We estimated the extraction ratio of creatinine per kidney as the difference between the arterial and venous concentration of creatinine divided by the arterial concentration and the APRC ratio as the difference between the venous and arterial concentration of APRC divided by the arterial concentration.

For each patient, we calculated the absolute asymmetry index for renal blood flow as:

$$
\frac{\mid \text { left MRBF }- \text { right MRBF | }}{(\text { MRBF left }+ \text { MRBF right)/2 }}
$$

In the absence of asymmetry in MRBF and renal artery stenosis, we expected a maximum difference between the left and the right kidney MRBF of approximately $25 \%$ (mean variability of two measurements in the same kidney plus two times the standard deviation), which is equal to a value of 0.22 of the asymmetry index. Therefore, we defined symmetry of MRBF as an asymmetry index $<0.22$ and asymmetry as an index $\geq 0.22$. In previous work, we calculated the asymmetry index in a slightly different way namely as flow in the kidney with the highest MRBF divided by that in the kidney with the lowest MRBF. ${ }^{6}$ The main advantage of the present asymmetry index is the linear scale, which enables its use in more advanced statistical models without the otherwise inevitable need for transformation. The cut-off value of 0.22 of the new index is equal to the previously applied 1.25 of the MRBF ratio.

Experimental data suggest that renal blood flow is not affected until the lumen is obliterated by at least $75 \%{ }^{1,2}$ This is comparable to a stenosis of approximately $50 \%$ as measured by 2-dimensional digital subtraction angiography. ${ }^{13}$ Therefore, in the analyses, patients were divided in three groups: no abnormalities (ARAS-), renal artery stenosis $<50 \%$ (low grade ARAS) and renal artery stenosis $\geq 50 \%$ (high grade ARAS).

\section{Statistical analysis}

Differences between the groups were analysed using either unpaired parametric t-tests or non-parametric tests or Pearson Chi-square tests. We explored the relationship between the absolute asymmetry index and factors with potential effect on renal blood flow further by forward stepwise linear regression analysis (with $p$ to enter set at 0.05 and $p$ for removal at 0.1 ). We considered: age, sex, body mass index, pulse pressure, mean arterial pressure, heart rate, serum creatinine, \%-stenosis and APRC ratios. Transformation of variables was applied if necessary. We used SPSS 11.5 for all statistical analysis. A p-value of $<0.05$ was considered statistically significant. Data with a normal distribution are presented as means $\pm S D$, otherwise medians and interquartile ranges are given. 


\section{Results}

Successful measurements of MRBF on both sides were available in 381 patients: 261 without abnormalities and 119 with renal artery stenosis. The latter group consisted of 59 patients with atherosclerotic unilateral stenosis (median percentage luminal reduction $44 \%$ (25-74)), 30 patients with atherosclerotic bilateral stenosis (median percentage luminal reduction 59\% (40-68)), 28 patients with fibromuscular dysplasia (FMD: 18 unilateral, 10 bilateral) and two patients with FMD on one side and atherosclerosis on the other. The analyses presented in this paper will be confined to the group of patients without abnormalities on renal angiography (ARAS-) and the group with unilateral atherosclerotic renal artery stenosis (ARAS+). Data of patients with FMD and bilateral atherosclerotic renal artery stenosis will be presented only for the comparison of the prevalence of asymmetry.

\section{Patient characteristics}

Patient characteristics are presented in Table 6.1. As expected, patients with ARAS+ were older, more often male and hypercholesterolemic. In addition they had higher pulse pressure, lower estimated creatinine clearance and more often manifestations of extra-renal atherosclerosis.

Table 6.1 Patient characteristics

$\begin{array}{lccc} & \begin{array}{c}\text { ARAS- } \\ \mathrm{n}=261\end{array} & \begin{array}{c}\text { Unilateral ARAS }+ \\ \mathrm{n}=59\end{array} & \mathrm{p} \\ & 50 \pm 13 & 62 \pm 9 & <0.0001 \\ \text { Age (years) } & 144 / 117 & 42 / 17 & 0.03 \\ \text { Sex (male/female) } & 28 \pm 5 & 27 \pm 4 & 0.34 \\ \text { Body mass index }\left(\mathrm{kg} / \mathrm{m}^{2}\right) & 59 & 70 & 0.10 \\ \text { Smoking } \% \text { ) } & 169 \pm 29 & 175 \pm 25 & 0.07 \\ \text { Office systolic blood pressure }(\mathrm{mmHg}) & 100 \pm 16 & 92 \pm 13 & <0.0001 \\ \text { Office diastolic blood pressure }(\mathrm{mmHg}) & 69 \pm 22 & 82 \pm 23 & <0.0001 \\ \text { Office pulse pressure (mmHg) } & 123 \pm 18 & 119 \pm 14 & 0.18 \\ \text { Office mean arterial pressure }(\mathrm{mmHg}) & 91 \pm 39 & 69 \pm 24 & <0.0001 \\ \text { Creatinine clearance (ml/min) } & 11 & 18 & 0.14 \\ \text { Diabetes mellitus }(\%) & 27 & 54 & <0.0001 \\ \text { Hypercholesterolemia (\%) } & 26 & 59 & <0.0001 \\ \text { Extra-renal atherosclerosis (\%) } & & & \end{array}$

Within the diagnostic groups, patients with symmetry (SYM) and asymmetry (ASYM) of flow did not differ substantially regarding patient characteristics. ASYM ARAS- subjects suffered somewhat less often from hypercholesterolemia (19\% vs. $33 \%, p=0.03)$ and extra-renal atherosclerosis $(18 \%$ vs. $33 \%, p=0.01)$ than the SYM ARAS-. ASYM ARAS+ had, on average, a higher body mass index than SYM ARAS+ (28 (27-32) vs. 25 (23$28), p=0.005)$. During the flow measurements blood pressure remained constant in all patient groups. 


\section{Prevalence of Asymmetry}

The prevalence of asymmetry was almost similar in the group without abnormalities $(45 \%)$ and in the group with unilateral renal artery stenosis (50\%). Subjects with FMD had less often apparent differences between the kidneys regarding flow (unilateral FMD $31 \%$, bilateral FMD 39\%) whereas subjects with bilateral atherosclerotic renal artery stenosis had the highest prevalence of asymmetry (62\%)

\section{Grade of luminal reduction and asymmetry}

A greater degree of luminal reduction coincided with a larger difference in MRBF between the kidney with the stenotic renal artery and the non-stenotic one. Yet, kidneys with unilateral ARAS did not always have a lower MRBF than the non-stenotic kidney. In fact, in half of the patients with stenosis $>50 \%$, blood flow per unit mass of tissue through the kidney on the side of the renal artery stenosis was similar $(43 \%)$ to or even higher $(7 \%)$ than that through the non-stenotic kidney. (Figure 6.1)

Figure 6.1 Asymmetry index in relation to lumen reduction (\%).

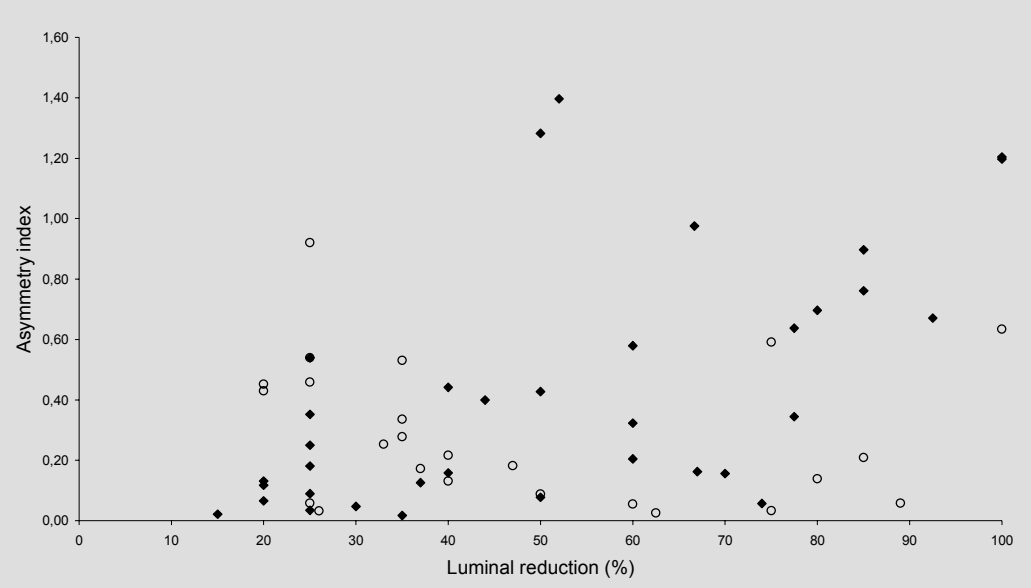

- MRBF of the kidney with unilateral ARAS < MRBF of the contralateral kidney; O MRBF of the kidney with unilateral ARAS > MRBF of the contralateral kidney. This figure depicts the grade of stenosis versus the asymmetry index.

\section{Split renal function}

Figure 6.2 presents MRBF, APRC ratio and creatinine ratio per kidney for the three diagnosis groups, ARAS- is depicted for reasons of comparison only. 
Figure 6.2 Split renal function.
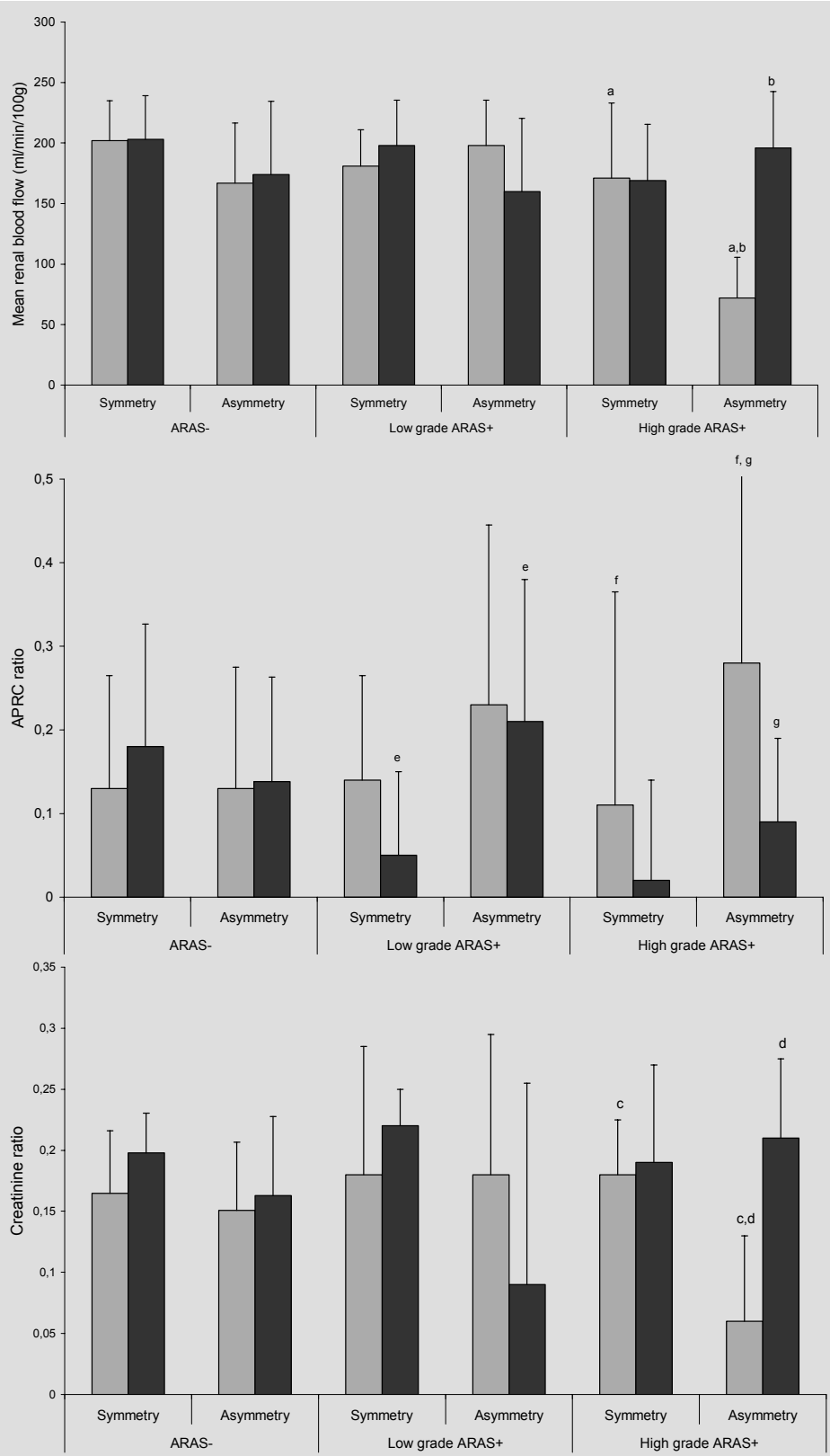

Values are presented as means and interquartile range. ${ }^{\mathrm{a}},{ }^{\mathrm{b}},{ }^{\mathrm{c}}$ and ${ }^{\mathrm{d}}$ denote $\mathrm{p}$-value $<0.05$ and ${ }^{\mathrm{e}}$, ${ }^{\mathrm{f}}$ and ${ }^{g}$ denote $p$-value $0.05-0.10$ for comparisons between (or within) groups. This figure shows MRBF (upper panel), APRC (middle panel) and creatinine ratio (lower panel) through kidneys with unilateral ARAS ( $\square$ ) and the non-stenotic kidney ( $\square)$ (or through left ( $\square$ ) and right ( $\square$ ) kidneys in ARAS-). 


\section{High grade unilateral ARAS}

ASYM had a lower MRBF through the stenotic kidney. APRC values differed to a great extent between patients. The APRC tended to be higher in the stenotic kidneys of ASYM as compared to SYM, the difference was, however, not statistically significant $(p=0.09)$. The extraction of creatinine was significantly lower in the stenotic kidneys of ASYM as compared to SYM. When the two kidneys of ASYM were compared, the stenotic kidney received less flow $(p=0.001)$, had a higher APRC ratio $(p=0.06)$ and extracted less creatinine $(p=0.03)$ than the non-stenotic kidney.

\section{Low grade unilateral ARAS}

Patients with low grade stenosis showed an intermediate pattern: patients with ASYM seemed, on average, to have slightly lower flow through the stenotic kidney and higher flow to non-stenotic kidney compared to SYM, but this was not statistically significant. The APRC ratio in the non-stenotic kidney was higher in ASYM $(p=0.09)$, but the extraction of creatinine was similar.

\section{Multivariable analysis}

In the group of patients without renal artery stenosis, only mean arterial pressure was weakly related to the asymmetry index. $\left(R^{2}=0.04, p=0.01\right)$. In the group of patients with renal artery stenosis, $28 \%$ of the variance of the asymmetry index was explained by three variables: body mass index, grade of stenosis (\%), the APRC ratio of the kidney contralateral to the stenosis $(p<0.0001)$.

\section{Clinical consequences of asymmetric perfusion}

Twenty-three out of the 28 patients with a high grade stenosis underwent renal revascularisation (two surgical reconstruction, 21 percutaneous transluminal renal angioplasty (with the insertion of a stent in 14). Judged by (immediate) postinterventional angiography films, PTRA was technically successful in all cases. Four patients who did not undergo PTRA were treated with medication only; in one of these because of occlusion of the vessel, in one because the non-stenotic kidney produced APRC whereas APRC production was depressed in the stenotic kidney, in one patient there was a good clinical response to medication and in one patient angiographic abnormalities had been judged to be not severe enough to impede blood flow the time of the angiography (whereas the panel of radiologists of this study judged differently). One other patient died before the scheduled date of the PTRA. During follow-up period, one patient died two days after surgical revascularisation and of one patient no blood pressure measurements were available during a visit to our the outpatient clinic while using medication. Analysis of the clinical outcome after three months, was, therefore, performed on data of 21 patients. Four patients underwent 
intervention less than a year ago, therefore the outcome after one year was evaluated in 17 patients. Figure 6.3 shows that blood pressure had decreased after the intervention.

Figure 6.3 Change of blood pressure after three months and one year after revascularisation.
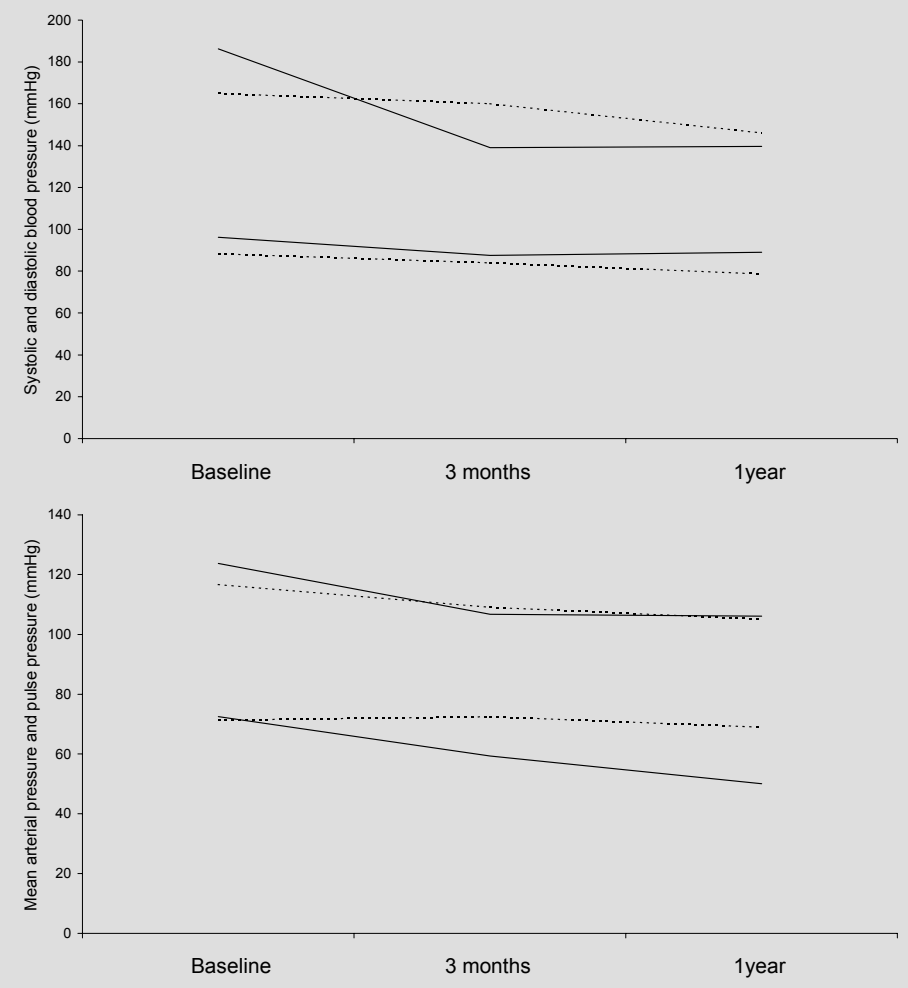

This figure shows blood pressure response (mean arterial pressure and pulse pressure) three months $(n=21)$ and one year $(n=17)$ after revascularisation in patients with symmetry $(-)$ and asymmetry (..........). Reduction of mean arterial pressure was similar between these two groups, however, pulse pressure only fell in patients with symmetric perfusion.

SYM and ASYM had about comparable reduction of the mean arterial pressure at three months $(16(-2-28)$ vs. $8(-3-13) \mathrm{mmHg}, \mathrm{p}=0.12)$ and at twelve months $(25(2-36)$ vs. 11 $(0-25) \mathrm{mmHg}, \mathrm{p}=0.34)$. However, in SYM the reduction in pulse pressure tended to be more pronounced than in ASYM both at three months (16 (4-27) vs. $-1(-10-15) \mathrm{mmHg}$, $p=0.07)$ and at twelve months $(20(6-40)$ vs. $3(-7-19), p=0.06)$ (Figure 6.3). The two groups did not differ with respect to the number of prescribed antihypertensive drugs. (SYM vs. ASYM: at baseline 2.0 (1.8-3.0) vs. 2.0 (2.0-2.0) drugs, at three months 2.0 (1.0-3.0) vs. 2.0 (2.0-3.0) drugs and at one year 2.0 (1.3-3.0) vs. 2.0 (2.0-3.0) drugs)). Possibly because of low numbers, none of the differences between responders and 
non-responders reached statistical significance threshold. Patients whose outcome was classified as failure were characterised by a higher asymmetry index $((0.53)(0.16-0.75)$ vs. $0.16(0.07-0.46), p=0.09)$ and a lower MRBF on the stenotic side (111 (63-162) vs. $154(107-260) \mathrm{ml} / \mathrm{min} / 100 \mathrm{~g}, \mathrm{p}=0.18)$ than responders. Non-stenotic MRBF was however similar (195 (133-222) vs. $194(125-242) \mathrm{ml} / \mathrm{min} / 100 \mathrm{~g}, \mathrm{p}=0.83)$. APRC ratios of nonresponders and responders were $0.27(0.08-1.23)$ and $0.12(-0.04-0.63)(p=0.23)$ in the stenotic kidney and $0.07(-0.01-0.14)$ and $0.03(-0.13-0.12)(p=0.32)$ in the non-stenotic kidney.

\section{Discussion}

The present study focused on the comparison of the two kidneys in the presence of unilateral atherosclerotic renal artery stenosis and hypertension. Higher grades of luminal reduction coincided with an increased difference in MRBF between the stenotic and the non-stenotic kidney. However, in half of the patients with high grade stenosis, flow through the kidneys was similar or even higher in the kidney with renal artery stenosis than in the contralateral one. Since we measured flow per 100 grams of kidney mass, it may very well be that, in absolute terms, patients with a symmetric flow have a clear-cut difference in absolute RBF between both kidneys when these have different volumes. Differences in flow in this study relate to actual differences of flow per unit of functional mass. Whether a reduction in flow is focal or more uniformly distributed throughout the kidney, cannot be determined from our data.

Most human research has focused less on differences between the stenotic and the contralateral kidney per patient than on average flow rates through the stenotic and contralateral kidneys in groups of patients with renovascular disease. For example, Kimura et al. ${ }^{14}$ found that the average RBF and GFR were lower in the unilaterally stenotic kidney and higher in the contralateral kidney compared to kidneys of patients without renal artery stenosis. Further, they calculated that the contralateral kidney must suffer from glomerular hypertension and hyperfiltration. In our study, perfusion rate through a kidney supplied by an artery with a high grade stenosis, was lower than the average value of the group of patients without renal artery stenosis. However, blood flow through the other kidney was similar to the average value of subjects without stenosis. This kidney extracted higher amounts of creatinine per $100 \mathrm{~g}$ of kidney mass than the stenotic one. Renal APRC ratio was higher in the stenotic than in the contralateral kidney (of borderline statistical significance). Hence, at first glance, our results are completely consistent with the common view on unilateral flow reduction in renal artery stenosis. ${ }^{14}$ At closer examination, however, this pattern was not seen in the half of the patients displaying symmetry of flow. Reasons for this do not become clear from our data since patients with and without symmetry were remarkable similar with respect to patient characteristics. Multivariable analysis revealed only the degree of 
stenosis, renin activity and BMI as independent predictors of the grade of asymmetry. Considering the central role of the renin-angiotensin system in the (initial) elevation of blood pressure in animals with lumen artery restriction, we hypothesise that the presence of (a)symmetry may be reflecting whether a renal artery stenosis is the origin of hypertension and renal dysfunction in a patient or just an innocent bystander. As previously reported, in the group of patients without any renal artery abnormalities, $45 \%$ presented with clear-cut asymmetry. This was, however, unrelated to renin activity, possibly suggesting a different etiology. ${ }^{6}$ Strikingly, patients with unilateral renal artery stenosis caused by fibromuscular dysplasia had the lowest prevalence of asymmetric perfusion (31\%). Unfortunately, the number of patients with FMD was too small to allow any meaningful statistical testing.

Also, few experimental studies have investigated differences between both kidneys per animal. Sigmon et al. ${ }^{15}$ studied differences in RBF between the kidneys of $2 \mathrm{~K}, 1 \mathrm{C}$ rats. They intended to induce unilateral renal artery stenosis of less than $80 \%$ by clipping the blood supply to one of the two kidneys of rats in a series of experiments. They used clips with different internal diameters. Due to the impossibility to accurately measure luminal reduction in rats, they calculated the weight-corrected ratio of the non-clipped to the clipped RBF as a functional index. They defined a ratio $\leq 1.25$ as mild stenosis and a ratio $>1.30$ as moderate stenosis. Both groups were hypertensive and had similar blood pressure, cardiac output and total peripheral resistance. Absolute flow through the clipped kidney and mass was always reduced four weeks after clipping. However, moderate stenotic rats had a 2.6 fold higher plasma renin activity fifteen minutes after endothelial nitric oxide synthase inhibition with L-NAME than mildly stenotic ones. They assumed that their functional classification paralleled anatomical luminal obstruction, despite the absence of any correlation between clip size and blood pressure or degree of stenosis. We found that even in patients with stenosis $>50 \%$, only the half showed a lower blood flow through the stenotic kidney than through the contralateral one. So, in the light of our findings, it is very tempting to interpret the data of Sigmon et al. on the absence of a clear-cut relation between clip size (and thus degree of lumen obstruction) and differences between the kidneys in renal blood flow, as, indeed, circumstantial evidence that the phenomenon we observed is also apparent in experimentally induced $2 \mathrm{~K} 1 \mathrm{C}$ hypertension. Interestingly, in more recent work they studied $2 \mathrm{~K} 1 \mathrm{C}$ hypertension in thirteen weeks old rats. Only $50 \%$ of the clipped rats survived until thirteen weeks and none of them presented with asymmetric perfusion pattern. ${ }^{16}$ They hypothesized that adaptation to a certain "normal" flow value, might be pivotal for rats in order to survive.

The results of renal revascularisation are disappointing in many patients with atherosclerotic renal artery stenosis. This has been the incentive for the search for predictors of a beneficial outcome. In this respect, the degree of renin activation and renal hemodynamics have received a lot of attention. Initially, a profound role for the determination of renin activity was suggested, ${ }^{17}$ but later studies have tempered this 
enthusiasm. ${ }^{18,19}$ In our study, (high) renin activity was a predictor of asymmetry. So, since asymmetric patients responded worse to revascularisation, if anything, renin activity was associated with worse outcome. This confirms findings from the group of Radermacher et al. who reported that an increased plasma renin activity was associated with a worsening of renal function after revascularisation, although this result did not sustain in multivariable analysis. ${ }^{20}$ For the renal hemodynamic variables, the discriminative abilities of flow through the stenotic kidney and the contralateral kidney have been studied. Volume-normalized blood flow through stenosed kidneys did not differ between responders and non-responders, ${ }^{21}$ whereas (absolute) blood flow through the kidney contralateral to unilateral stenosis was on average found to be lower in responders, although with considerable overlap between categories. ${ }^{22}$ However, the interpretation of the latter study is complicated. In that study, only patients with either an asymmetric scintigram or an (unilateral) abnormal excretatory urogram were subjected to intra-arterial angiography. Further, in $20 \%$ of their patients surgical revascularisation was performed because of segmental renal artery stenosis (as diagnosed by functional studies) in the absence of (main) renal artery stenosis on intra-arterial angiography. Therefore, selection bias may have been present. In the present study, responders and non-responders did differ with respect to flow through the kidney supplied by an artery with (high grade) stenosis, however this result was not statistically significant. Considering the great interindividual variability of renal blood flow between patients with hypertension, ${ }^{23}$ the low predictive value does not seem very surprising. It has been known for a long time that an unilateral decrease in perfusion pressure lead to changes in hemodynamics and volume not only in the ipsilateral but also in the contralateral kidney. Furthermore, it has been suggested that the contralateral kidney is exposed to systemic hypertension, while the kidney on the stenotic side is "protected" by the stenosis. ${ }^{24,25}$ Whether the kidneys are adapting to these changes may be reflected by differences with respect to volume-corrected flow between the kidneys. We found that asymmetry of renal blood flow may have adverse functional consequences since blood pressure responses to revascularisation seemed to be less marked in patients with asymmetry than in patients with a symmetric perfusion pattern. Findings from other groups studying indices of both kidneys, lend some support to this hypothesis. Studies with captopril renography found that severe asymmetry regarding relative clearance values by the kidneys was more common in non-responders than in responders, ${ }^{18}$ and if both kidneys display a (very) high intra-renal resistance, the change of a favourable outcome after renal revascularisation is low. ${ }^{20}$

\section{Conclusion}

In patients with renal artery stenosis, asymmetry of renal blood flow was not as consistently present as commonly assumed and neither necessarily at the expense of the stenotic kidney. Asymmetry was associated with functional consequences: higher renin activation, lower extraction of creatinine and a less favorable outcome after 
revascularisation. Asymmetric perfusion, rather than the stenosis per se, may be associated with adverse functional consequences. Further studies into mechanisms of asymmetry of renal blood flow may provide new insights for the role of renal revascularisation in atherosclerotic renal artery stenosis. 


\section{References}

1. Schoenberg SO, Bock M, Kallinowski F, Just A. Correlation of hemodynamic impact and morphologic degree of renal artery stenosis in a canine model. J Am Soc Nephrol. 2000; 11:2190-8.

2. Imanishi M, Akabane S, Takamiya M, Kawamura M, Matsushima Y, Kuramochi M, Omae T. Critical degree of renal arterial stenosis that causes hypertension in dogs. Angiology. 1992;43:833-42.

3. Lerman LO, Schwartz RS, Grande JP, Sheedy PF, Romero JC. Noninvasive evaluation of a novel swine model of renal artery stenosis. J Am Soc Nephrol. 1999;10:1455-65.

4. Schreij G, Ritsema GH, Vreugdenhil G, de Leeuw PW. Stenosis and renographic characteristics in renovascular disease. J Nucl Med. 1996;37:594-7.

5. Vasbinder GBC, Nelemans PJ, Kessels AG, Kroon AA, De Leeuw PW, Van Engelshoven JM. Diagnostic tests for renal artery stenosis in patients suspected of having renovascular hypertension: a meta-analysis. Ann Intern Med. 2001;135:401-11.

6. van Onna M, Houben AJ, Kroon AA, Wierema TK, Koster D, van Engelshoven JM, de Leeuw PW. Asymmetry of renal blood flow in patients with moderate to severe hypertension. Hypertension. 2003;41:108-13.

7. Blaufox MD, Fromowitz A, Gruskin A, Meng CH, Elkin M. Validation of use of xenon 133 to measure intrarenal distribution of blood flow. Am J Physiol. 1970;219:440-444.

8. Hollenberg NK, Mangel R, Fung HY. Assessment of intrarenal perfusion with radioxenon: a critical review of analytical factors and their implications in man. Semin NuCl Med. 1976; 6:193-216.

9. de Leeuw PW, Birkenhager WH. Use of the xenon-133 washout technique in diagnosing renovascular disease in hypertension. Nephron. 1986;44:5-7.

10. Wierema TK, Houben AJ, Kroon AA, Koster D, van der Zander K, van Engelshoven JM, de Leeuw PW. Nitric oxide dependence of renal blood flow in patients with renal artery stenosis. J Am Soc Nephrol. 2001;12:1836-43.

11. Simon D, Hartmann DJ, Badouaille G, Caillot G, Guyenne TT, Corvol P, Pau B, Marchand J. Two-site direct immunoassay specific for active renin. Clin Chem. 1992;38:1959-62.

12. Cockcroft DW, Gault MH. Prediction of creatinine clearance from serum creatinine. Nephron. 1976;16:31-41.

13. May AG, Van de Berg L, DeWeese JA, Rob CG. Critical artery stenosis. Surgery. 1963; 54:250-9.

14. Kimura G, London GM, Safar ME, Kuramochi M, Omae T. Glomerular hypertension in renovascular hypertensive patients. Kidney Int. 1991;39:966-72.

15. Sigmon DH, Beierwaltes $\mathrm{WH}$. Degree of renal artery stenosis alters nitric oxide regulation of renal hemodynamics. J Am Soc Nephrol. 1994;5:1369-77.

16. Sigmon DH, Beierwaltes WH. Influence of nitric oxide in the chronic phase of two-kidney, one clip renovascular hypertension. Hypertension. 1998;31:649-56.

17. Vaughan ED, Jr., Buhler FR, Laragh JH, Sealey JE, Baer L, Bard RH. Renovascular hypertension: renin measurements to indicate hypersecretion and contralateral suppression, estimate renal plasma flow, and score for surgical curability. Am J Med. 1973;55:402-14.

18. Helin KH, Lepantalo M, Edgren J, Liewendahl K, Tikkanen T, Tikkanen I. Predicting the outcome of invasive treatment of renal artery disease. J Intern Med. 2000;247:105-10.

19. Rossi GP, Cesari M, Chiesura-Corona M, Miotto D, Semplicini A, Pessina AC. Renal vein renin measurements accurately identify renovascular hypertension caused by total occlusion of the renal artery. J Hypertens. 2002;20:975984.

20. Radermacher J, Chavan A, Bleck J, Vitzthum A, Stoess B, Gebel MJ, Galanski M, Koch KM, Haller $\mathrm{H}$. Use of Doppler ultrasonography to predict the outcome of therapy for renal-artery stenosis. N Engl J Med. 2001;344:410-7.

21. Binkert CA, Debatin JF, Schneider E, Hodler J, Ruehm SG, Schmidt M, Hoffmann U. Can MR measurement of renal artery flow and renal volume predict the outcome of percutaneous transluminal renal angioplasty? Cardiovasc Intervent Radiol. 2001;24:233-9. 
22. Palmer JM. Prognostic value of contralateral renal plasma flow in renovascular hypertension. Analysis of 55 surgically treated patients with proved unilateral lesions. Jama. 1971;217: 794-802.

23. Hollenberg NK, Adams DF. The renal circulation in hypertensive disease. Am $J$ Med. 1976;60:773-84.

24. Ubara Y, Hara S, Katori H, Yamada A, Morii H. Renovascular hypertension may cause nephrotic range proteinuria and focal glomerulosclerosis in contralateral kidney. Clin Nephrol. 1997;48:220-3.

25. Tullis MJ, Zierler RE, Caps MT, Bergelin RO, Cantwell-Gab K, Strandness DE, Jr. Clinical evidence of contralateral renal parenchymal injury in patients with unilateral atherosclerotic renal artery stenosis. Ann Vasc Surg. 1998;12:122-7. 


\section{Chapter}

The effect of restoring renal patency on renal function in patients atherosclerotic renal artery stenosis. A systematic review 


\section{Abstract}

The goals of restoration of renal artery patency are usually two-fold: to improve blood pressure control and to stabilize (or even improve) renal function. The effect on renal function is, however, largely unknown since results of studies are conflicting, possibly as a result of small study sizes and differences in study design and patient selection. The large number of studies that have been published raises the opportunity to study by meta-anlysis the effect of intervention on renal function in a large congregate of patients. A comprehensive search of MEDLINE and EMBASE was undertaken to identify all studies that evaluated the effect of renal patency restoration on serum creatinine in patients with hypertension and renal artery stenosis with a clinical follow-up of at least three months. Although average change of creatinine after intervention differed greatly between the individual studies, serum creatinine concentration after intervention fell by $5 \mu \mathrm{mol} / / \mathrm{l}(p=0.04)$. Renal patency restoration in patients with atherosclerotic renal artery stenosis and hypertension may lead to a modest improvement of renal function. Whether this effect has clinical relevance is uncertain. 


\section{Introduction}

Atherosclerotic renal artery stenosis (ARAS) is associated with hypertension and renal dysfunction and is a progressive over time. In a study examining the natural history of ARAS by duplex scanning, $49 \%$ of arteries with high grade ARAS ( $\geq 60 \%$ stenosis) showed progressive reduction of the arterial lumen and $56 \%$ of the arteries with low grade ARAS (<60\% stenosis) progressed to the category of high grade stenosis within three years despite of the use of antihypertensive medication. ${ }^{1}$ The goals of restoration of renal artery patency, therefore, are usually two-fold: to improve blood pressure control and to stabilize (or even improve) renal function. Since the introduction of PTRA, a large number of (mainly observational) studies has been published on the clinical outcome after various forms of intervention. The results of these studies are, however, conflicting, possibly as a result of small study sizes and differences in study design and patient selection. Nevertheless, the availability of these studies raises the opportunity to asses by meta-analysis the effect of intervention on renal function in a large congregate of patients in a longer time frame. Here we report on the results of such a metaanalysis. Further this meta-analysis aimed to explore which factors determined differences in outcome between studies.

\section{Materials and methods}

\section{Search strategy}

A comprehensive search of MEDLINE and EMBASE was performed using the following terms: (hypertension, renovascular OR reno* hypertension* OR renal artery obstruction OR renal artery stenosis OR atherosclerotic renal disease OR atherosclerotic renovascular disease) AND (PTRA OR percutaneous transluminal renal angioplasty OR angioplasty, balloon OR stent* $\mathrm{OR}$ vascular surgical procedure $\mathrm{OR}$ surgical revascularisation $\mathrm{OR}$ revascularisation $\mathrm{OR}$ revascularisation). No starting date was specified and the search was updated until February $1^{\text {th }} 2005$. Only studies published in English, German, French or Dutch were selected. Abstracts of the selected manuscripts were screened for inclusion- and exclusion criteria. If relevant, or if the abstract did not contain sufficient information to decide about the relevance, the full text of the article was retrieved. Further, the reference lists of the obtained articles were screened for potentially eligible studies. 


\section{Study selection}

Studies were included in the meta-analysis when they met the following criteria:

1. The intervention consisted of either surgical revascularisation or percutaneous transluminal renal angioplasty with/without the insertion of a stent, or a combination of these.

2. The inclusion of patients (whether prospective or retrospective) was consecutive and thus the study population did not represent a subgroup of any kind.

3. Absolute creatinine concentrations or calculated creatinine clearances and the appropriate standard deviation values measured before and after intervention were given, or these values could be derived from the data or obtained on written request to the corresponding author.

4. Follow-up after the intervention was at least three months.

5. At least $95 \%$ of the patients was hypertensive (blood pressure $\geq 140 / 90$ or the use of antihypertensive medication) before intervention.

\section{Studies were excluded when:}

1. In $\geq 5 \%$ of the cases, renal artery stenosis was

- detected incidentally during angiography for other reasons than suspicion of renovascular hypertension, and/or

- of non-atheromatous etiology, and/or

- caused by re-stenosis of previously successfully treated lesion(s), and/or

- located in a transplant kidney

2. The report consisted of less than 20 patients who completed follow-up of three months

3. A more recent study of the same researcher or research group was available, unless it was beyond doubt that a different patient sample had been used.

\section{Data extraction}

Relevant data from included studies were abstracted by two of us by use of a standardized form. If available, data were abstracted regarding patient source, (primary) indication for intervention, lesion characteristics, the applied intervention(s), renal function (creatinine or calculated creatinine clearance (with appropriate standard deviation(SD))), and potential confounders (age, gender, presence of diabetes mellitus, blood pressure and body weight). Discrepancies in the extracted data were resolved by the two authors examining the articles together.

\section{Assessment of study quality}

The quality of the studies was evaluated by use of the checklist by Downs and Black that allows the assessment of both randomised and non-randomised studies. ${ }^{2}$ This 
score assesses reporting, external validity, bias, confounding and power. With respect to power, this score needs to be customized. Since it would take 170 patients in order to detect a difference of serum creatinine $\geq 5 \mu \mathrm{mol} / \mathrm{l}$, (assuming a SD of 20, $\alpha=0.05$ and $1-\beta=0.90), 0$ points were given when the number of patients at follow-up was less than 169, 1 point when between 170 and 339,2 points between 340 and 509,3 points between 510 and 679,4 points between 680 and 849 and 5 points when more than 850 patients were included. We omitted items that were not applicable to any of the studies (numbers 1, 10, 14, 18, 21, 22 and 25). Quality was assessed by two of us and in case of disagreement discussed until consensus was reached.

\section{Data synthesis and analysis}

For each study, the difference (Diff) in average creatinine at baseline $\left(\mathrm{C}_{0}\right)$ and average creatinine at twelve months after intervention $\left(C_{1}\right)$ was calculated as: Diff $=C_{0}-C_{1}$. If no measurement at twelve months was provided we used, in order of preference, the measurement after six, three or 24 months. The SE of Diff was calculated as:

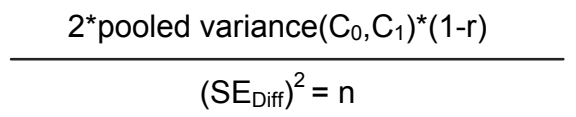

Where $\mathrm{n}$ is the number of patients, (when number of patients at baseline did not equal the number of patients at follow-up, the average of that was used) and with $r$ the correlation between $\mathrm{C}_{0}$ and $\mathrm{C}_{1}$. Since this correlation is generally not reported, this was estimated to approximate the intra-individual day-to-day variability ${ }^{3}$, and a value of $0.9^{4,5}$ was imputed for all studies. In two studies ${ }^{6,7}$ SE in stead of SD was reported and in these cases, SD was calculated as SE * $\sqrt{ }$. In two studies in which standard deviations were not provided, we imputed the SD of another (included) study that did provide SD and was most comparable with respect to number of participants and methodology. ${ }^{8,9}$ It should be noted that imputation of SD affects the weight of the study but not the effect measure.

The overall effect of intervention on renal function was calculated as the weighted average of the Diff of all studies by the inverse variance weight method. The presence of heterogeneity between studies was assessed by the $Q$ statistic ( $p$-value for significance: 0.1 ) and the forest plot. In case of heterogeneity between studies we applied a random effects model, otherwise a fixed effect model was used. The following potential sources of heterogeneity were considered upfront: publication year, type of intervention, lesion characteristics (unilateral vs. bilateral, ostial vs. non-ostial disease), certain patient characteristics (age, blood pressure, presence of diabetes mellitus), $\%$ lost to follow up). The amount of missing data prevented the sensible application of a mixed effect model with potential sources of heterogeneity as covariables. ${ }^{10}$ Therefore, 
heterogeneity was further explored through stratified analysis. Analyses were performed with StataSE 8.0.

\section{Results}

We identified 2768 published studies and 190 among these were potentially eligible after screening the abstract and the full text of these articles was obtained. Of twelve papers, we could not retrieve the full text. We approached the corresponding authors of 21 manuscripts for additional information, of whom nine responded and six were able to supply the requested data. The present analysis includes 28 studies. Table 7.1 provides reasons for exclusion of studies. A complete reference list of the excluded papers can be obtained from the authors. The most frequent cause for rejection of a patients series was that the effect of intervention on renal function was not reported in sufficient detail to allow pooling for this analysis. Usually, the more recent studies described the renal outcome, but expressed the effect either as percentage of all patients who had benefited from the intervention (which was not uniformely defined), or only in patients with pre-existing renal failure. Older studies usually did not evaluate the effect on renal function.

Table 7.1 Reasons for exclusion.

Reason for exclusion (not mutually exclusive) Number of publications (\%)

\begin{tabular}{lc}
\hline No consecutive inclusion of patients & $23(14)$ \\
Renal function was not reported in sufficient detail, or only for & $90(56)$ \\
subgroups & $10(6)$ \\
Duration of follow-up less than three months & $6(4)$ \\
At least $5 \%$ of patients was normotensive & $20(12)$ \\
At least $5 \%$ of the patients had any of the following: & \\
$\quad$ - accidentally detected renal artery stenosis & \\
- non-atheromateous etiology & \\
- re-stenosis of previously successfully treated lesion(s) & $5(3)$ \\
- stenosis in a transplant kidney & $12(7)$ \\
A more recent study was available from the same research group & $10(6)$ \\
Lull text article could not be retrieved & $6(4)$ \\
Article did not report a patient series of revascularization & \\
Total & 162
\end{tabular}

\section{Characteristics of studies}

Characteristics of patients and procedures and the outcome of the included studies are summarized in Table 7.2. 
Table 7.2 Characteristics of included studies.

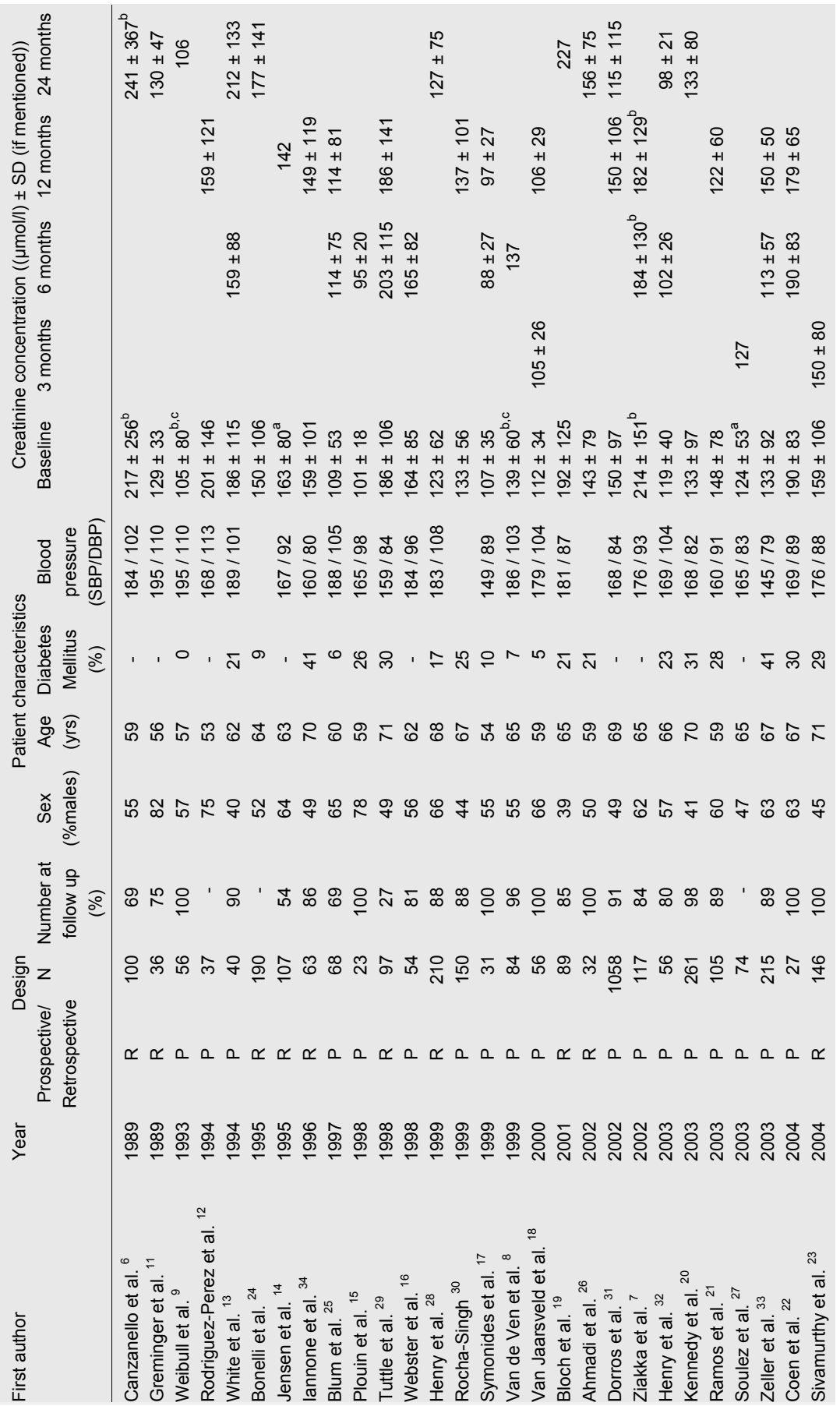


(continued)

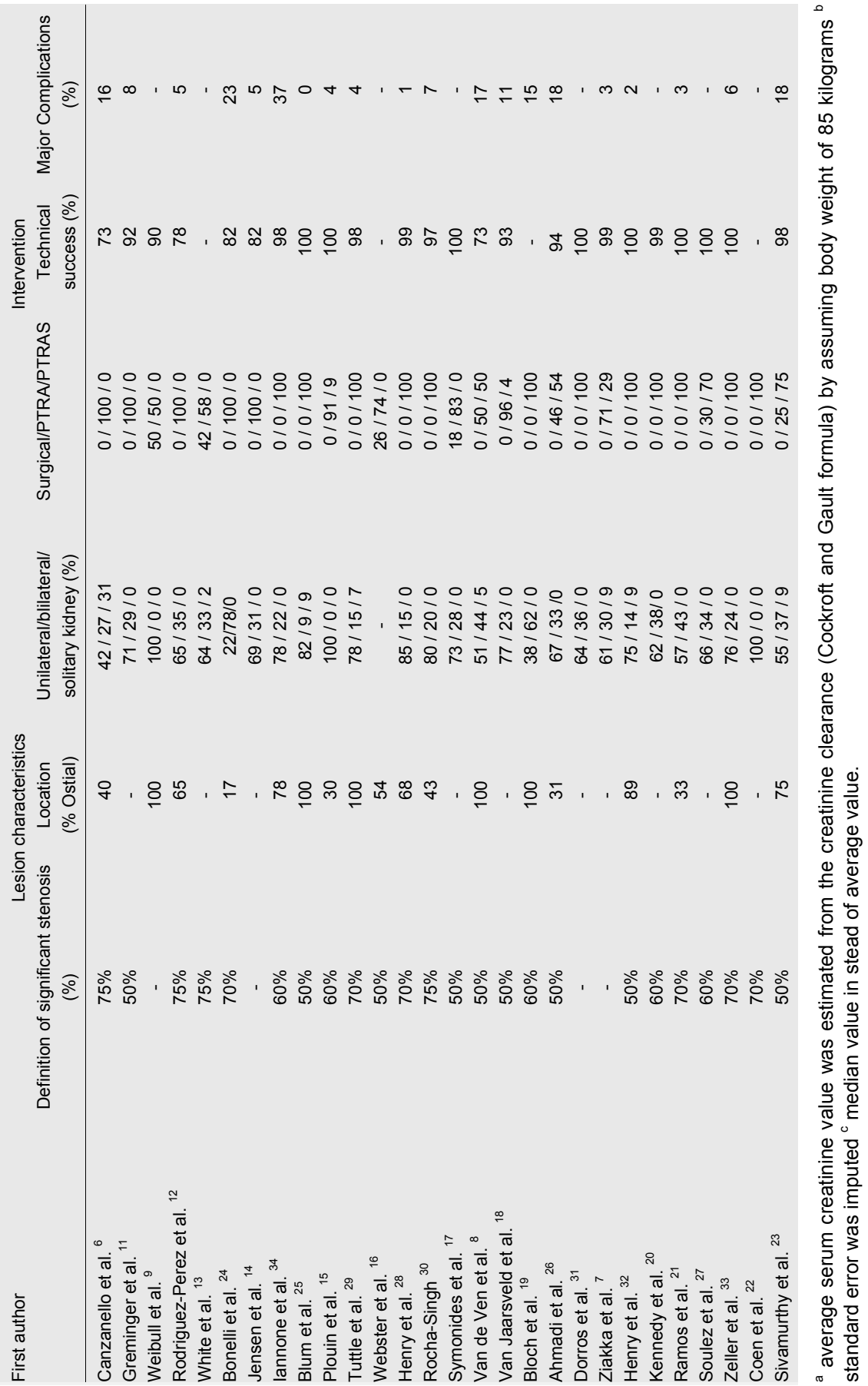




\section{Setting}

All but three studies were performed in departments of internal medicine/ nefrology ${ }^{6,8,11-22}$ or radiology. ${ }^{23-27}$ In five studies this information was lacking. ${ }^{28-33} \mathrm{We}$ qualified three studies as $\mathrm{RCT},{ }^{8,15,18}$ fifteen as prospective observational $^{7,9,12,13,16,17,20-22,25,27,30-33}$ (among which the study by Webster and all. ${ }^{16}$ which is sometimes refered to as a RCT eventhough not all patients were randomised) and ten as retrospective studies. $6,11,14,19,23,24,26,28,29,34$

\section{Patients}

The average age of the patients in the included studies varied between 53 and 71 years. In most reports males and females were about evenly presented, although in ten $65 \%$ (or more) of the participants were male., $, 111,12,14,15,18,25,29,32$ Average serum creatinine before intervention varied from high-normal to evidently increased (101-216 $\mu \mathrm{mol} / \mathrm{l})$. According to the average blood pressure values at baseline, blood pressure was reasonably controlled $(R R<160 / 90)$ in four studies, ${ }^{17,29,33,34}$ in all others, however, blood pressure was markedly elevated. The indication for the intervention was usually hypertension ${ }^{13,15,16,19}$ or a combination of hypertension and the prevention of renal function decline; ${ }^{6,17,18,20,23,28,31,33}$ in three studies a minority of patients $(9-23 \%)$ underwent intervention because of flash pulmonary edema. ${ }^{20,23,31}$ The prevalence of diabetes mellitus varied between 0 and $41 \%$; rather remarkably, in nearly $25 \%$ of the included studies the prevalence of this main competitive explanation of renal function deterioration was not reported. $6,7,11,12,14,16,27,31$

\section{Lesion characteristics}

In all studies intervention was done only when the renal artery lumen was reduced by at least $50 \%$. The actual cut-off, however, varied between 50 and $75 \%$, and in seven cases additional abnormalities were required like a certain renal artery pressure gradient $^{19-21,30,34}$ or some indication of decrease of function of the affected kidney. ${ }^{15,33}$ Three studies excluded bilateral disease, ${ }^{9,15,22}$ all others included both patients with unilateral and bilateral renal artery stenosis, the prevalence of bilateral disease varying between 6 and $62 \%$. Intervention of renal artery stenosis in a solitary kidney was not often applied in most series (0-9\%), with the exception of the study by Canzanello et al. in which in $31 \%$ of the participants a procedure in diseased renal artery supplying a solitary kidney was performed. ${ }^{6}$ In thirteen out of the nineteen series that subdivided lesions according to their location within the artery (ostial vs. non-ostial), ostial lesions were more prevalent. 


\section{Procedures}

The procedures applied in the series were PTRA in four, $6,11,12,14$ PTRA plus stenting in six, ${ }^{21,25,29,31,33,34}$ primary stenting in five, ${ }^{19,20,22,30,32}$ PTRA or primary stenting or a combination of PTRA and stenting in eight ${ }^{7,8,15,18,23,26,27,32}$ and PTRA or surgery in four studies. $^{9,16,17,35}$ Primary success rate was below $85 \%$ in four reports ${ }^{6,8,12,24}$ and above $90 \%$ in the rest, exceeding $95 \%$ in the majority of these. The definition of primary success differed widely presumably affecting the reported rates. Restenosis has been actively looked for in thirteen out of the 28 studies, and was present in $11-48 \%$ of the cases. Again, also the definition of restenosis varied. Adverse event rates were reported to be $15 \%$ or less, three studies excepted with rates of $17 \%{ }^{8}, 23 \%{ }^{34}$ and $29 \%{ }^{24}$

\section{Follow-up measurements}

Follow-up measurements were performed at regular intervals in 21 studies $^{7-9,12-18,20,22,23,25,27,29-34}$ and only at begin and end in seven studies. . $^{6} 11,19,21,24,26,28$ Two studies explicitly mentioned only to have performed follow-up measurements in patients in whom the intervention was qualified as technically satisfactory. ${ }^{6,21}$ The percentage of participants in which follow-up was obtained was rather low $(<85 \%)$ in many studies. $6,7,11,14,16,25,29,32$ Even more distressingly, a number of studies did not mention the number at follow-up at all, or only provided values of participants that had completed follow-up. ${ }^{12,24,27}$

\section{Quality of study}

The quality of studies varied a great deal, with a median quality score of twelve (interquartile range 11-14). (Table 7.3). None of the studies obtained the maximum score of 25 , mainly because of loss of points in the categories "internal validity-bias" (which contained issues like blinding , statistical analysis and outcome measurement), "internal validity-confounding" (which contained items on selection and allocation of patients, confounding and (handling of) lost-to follow up) and "power". All three RCT's obtained scores above the median.

\section{Clinical outcome}

The average effect of intervention on renal function as estimated by random effect modelling was a decrease of serum creatinine concentration by $5(0-10) \mu \mathrm{mol} / / \mathrm{l}$ (Figure 7.1). However, the change of creatinine after intervention differed greatly between the individual studies and the $Q$ statistic provided evidence of heterogeneity between studies $(p=0.01)$. The effect of study characteristics on the effect of intervention is shown in Figure 7.2. The length of the follow-up period and study design (prospective vs. retrospective) seemed to affect the estimation of the effect of intervention on renal function. However, since in our dataset retrospective studies usually had a longer follow-up period, it is impossible to untangle these two effects. Renal function at 
baseline (serum creatinine above or below median of $143 \mu \mathrm{mol} / \mathrm{l}$ ), type of intervention (in $\geq 60 \%$ of the patients PTRA only vs. $\geq 60 \%$ of the patients primary stenting or PTRAassisted stenting), publication year (before or after the median year 1998), number of participants (more or less than the median number of 62), and study quality (more or less than the median score of twelve) seemed to have no effect on the estimation, although the precision of the estimation seemed higher in studies with a higher quality score. Because of incomplete figures on prevalence of diabetes mellitus, lesion characteristics (ostial vs. non-ostial disease, unilateral vs. bilateral involvement) and restenosis, we were unable to investigate the role of these variables in heterogeneity of the effect size.

Table 7.3 Quality Index Score.

\begin{tabular}{|c|c|c|c|c|c|c|}
\hline \multirow[t]{2}{*}{ Author } & \multirow[t]{2}{*}{ Reporting } & \multirow{2}{*}{$\begin{array}{l}\text { External } \\
\text { validity }\end{array}$} & \multicolumn{2}{|c|}{ Internal validity } & \multirow[t]{2}{*}{ Power } & \multirow{2}{*}{$\begin{array}{l}\text { Overall } \\
\text { score }\end{array}$} \\
\hline & & & bias & confounding & & \\
\hline Canzanello et al. ${ }^{6}$ & 6 & 1 & 2 & 0 & 0 & 19 \\
\hline Greminger et al. $^{11}$ & 5 & 1 & 2 & 0 & 0 & 8 \\
\hline Weibull et al. $^{9}$ & 9 & 0 & 3 & 2 & 0 & 14 \\
\hline Rodriguez-Perez et al. ${ }^{12}$ & 6 & 3 & 1 & 0 & 0 & 10 \\
\hline White et al. ${ }^{13}$ & 6 & 0 & 3 & 0 & 0 & 9 \\
\hline Bonelli et al. $^{24}$ & 8 & 3 & 2 & 0 & 0 & 13 \\
\hline Jensen et al. ${ }^{14}$ & 6 & 1 & 2 & 0 & 0 & 9 \\
\hline lannone et al. ${ }^{34}$ & 7 & 3 & 2 & 0 & 0 & 12 \\
\hline Blum et al. ${ }^{25}$ & 6 & 1 & 2 & 0 & 0 & 9 \\
\hline Plouin et al. ${ }^{15}$ & 9 & 2 & 4 & 2 & 0 & 17 \\
\hline Tuttle et al. $^{29}$ & 6 & 2 & 3 & 0 & 0 & 11 \\
\hline Webster et al. ${ }^{16}$ & 7 & 1 & 4 & 0 & 0 & 12 \\
\hline Henry et al. ${ }^{28}$ & 8 & 3 & 2 & 0 & 1 & 14 \\
\hline Rocha-Singh. ${ }^{30}$ & 7 & 3 & 2 & 0 & 0 & 12 \\
\hline Symonides et al. ${ }^{17}$ & 8 & 0 & 3 & 1 & 0 & 12 \\
\hline Van de Ven et al. ${ }^{8}$ & 8 & 2 & 4 & 2 & 0 & 16 \\
\hline Van Jaarsveld et al. ${ }^{18}$ & 9 & 2 & 4 & 2 & 0 & 17 \\
\hline Bloch et al. ${ }^{19}$ & 5 & 3 & 2 & 0 & 0 & 10 \\
\hline Ahmadi et al. ${ }^{26}$ & 7 & 3 & 2 & 1 & 0 & 13 \\
\hline Dorros et al. ${ }^{31}$ & 6 & 3 & 2 & 0 & 5 & 16 \\
\hline Ziakka et al. $^{7}$ & 6 & 3 & 2 & 0 & 0 & 11 \\
\hline Henry et al. ${ }^{32}$ & 8 & 0 & 3 & 0 & 0 & 11 \\
\hline Kennedy et al. ${ }^{20}$ & 6 & 2 & 3 & 1 & 1 & 13 \\
\hline Ramos et al. ${ }^{21}$ & 9 & 1 & 2 & 1 & 0 & 13 \\
\hline Soulez et al. ${ }^{27}$ & 6 & 1 & 3 & 1 & 0 & 11 \\
\hline Zeller et al. ${ }^{33}$ & 9 & 2 & 4 & 1 & 1 & 17 \\
\hline Coen et al. ${ }^{22}$ & 7 & 2 & 3 & 0 & 0 & 12 \\
\hline Sivamurthy et al. ${ }^{23}$ & 9 & 3 & 3 & 1 & 0 & 16 \\
\hline Maximum obtainable & 9 & 3 & 5 & 3 & 5 & 25 \\
\hline Median (IQR) & $7(6-8)$ & $2(1-3)$ & $3(2-3)$ & $0(0-1)$ & $0(0-0)$ & $12(11-14)$ \\
\hline
\end{tabular}


Figure 7.1 The change of creatinine after intervention.

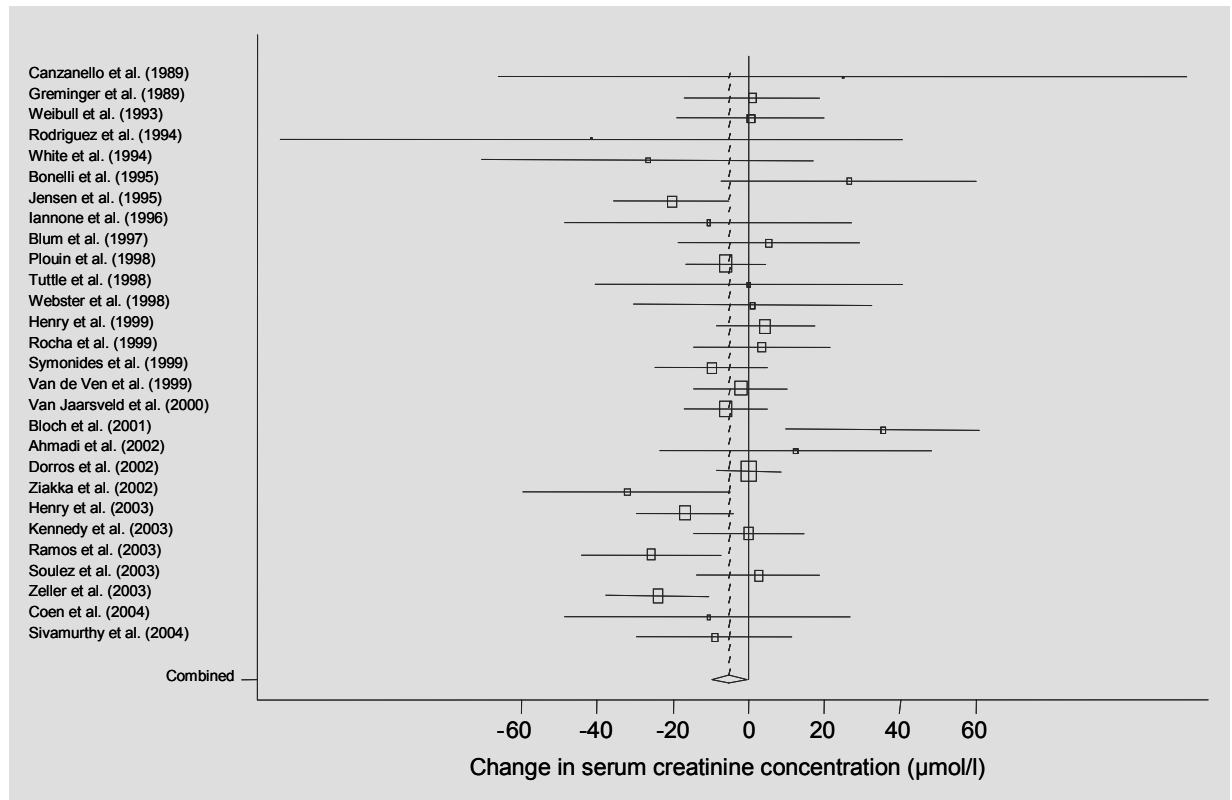

Figure 7.2 Influence of study characteristics on the estimation of the effect of renal outcome after intervention.

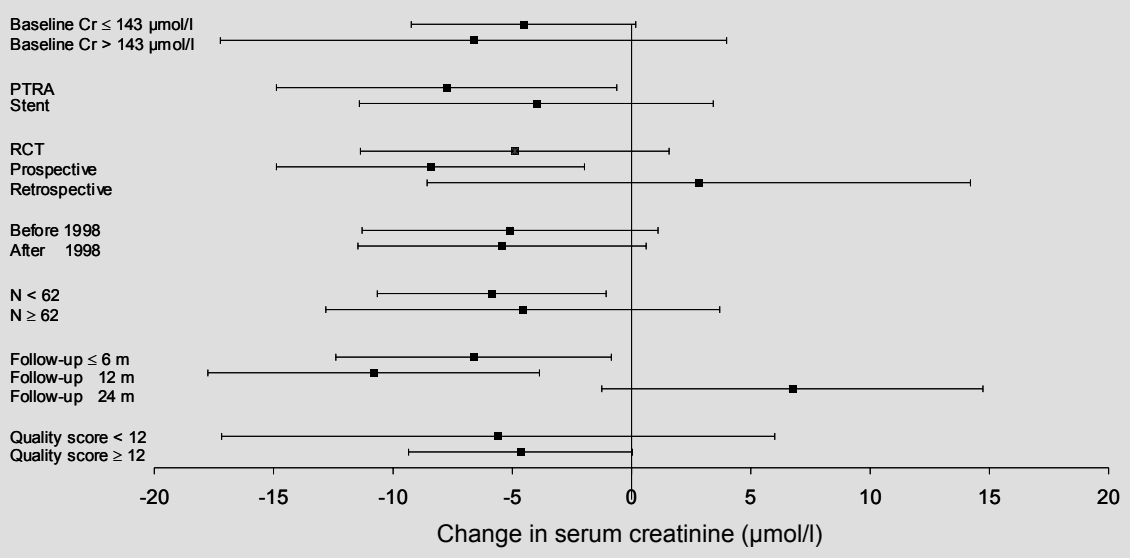

Unfortuanately, creatinine clearance was estimated in only ten studies and could therefore not be investigated. In Figure 7.3 the effect size (difference in creatinine) is plotted against the standard error, a so-called the funnel plot, which aims to investigate 
the likelihood of publication bias. Considering the symmetric appearance of the "funnel", the chance of (important) publication bias seems low.

Figure 7.3 Funnel plot.

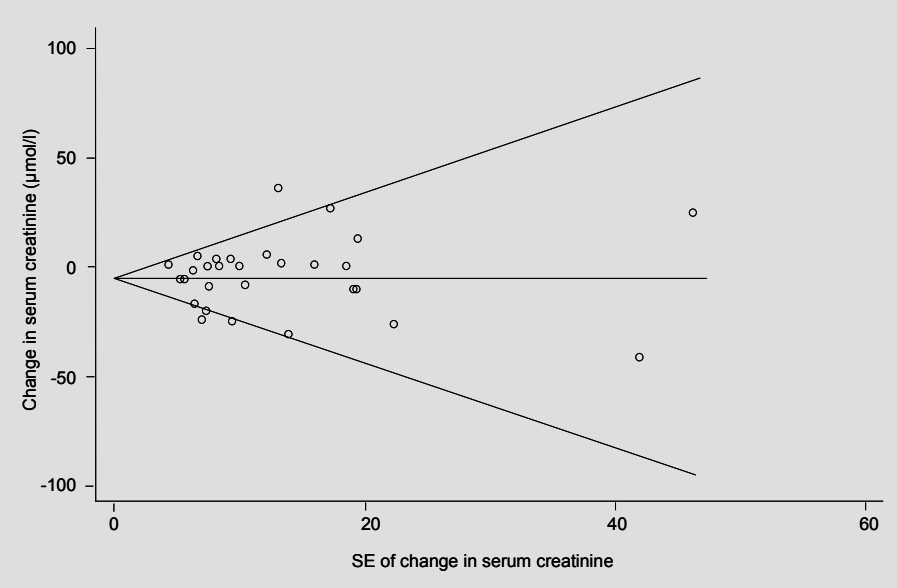

The effect size (difference in creatinine) is plotted against the standard error of the effect size with pseudo $95 \%$ confidence limits.

\section{Discussion}

Since the introduction of renal artery patency restoration, a large number of observational studies have been published on the clinical outcome after various interventions. Salvage of renal function is a relatively new aim of revascularisation and was often not evaluated in older series. Many studies that did evaluate renal function and were potentially suitable for our meta-analysis, reported data in a way that prevented evaluation of change of serum creatinine. Review of 28 published series, with a total of 3582 subjects, indicated that renal patency restoration in patients with atherosclerotic renal artery stenosis and hypertension may lead to a small, yet statistically significant, improvement of renal function. The clinical relevance of this benefit, if any, remains to be established.

In order to value our findings some methodological issues need to be discussed. Firstly, in any meta-analysis point estimates of the effect size from the included studies will differ. This can occur by "sampling error" (i.e. the true effect is the same in the each study), but usually variability exceeds that expected from sampling error only. In the latter case, effect size estimates are said to be "heterogeneous". Heterogeneity in systematic reviews may occur because of differences, artefactual or real, in treatment 
effect across the different studies. Whereas artefactual differences only hamper interpretation, real differences provide the possibility of studying effect-modification. In the present report, heterogeneity proved to be non-negligible despite stringent inclusion criteria. Presumably, this should be attributed, at least in part, to artefactual differences, since there was substantial variation in baseline renal function, lesion characteristics, procedures, follow-up period and numbers lost to follow up. Subgroup analyses provided no convincing evidence that type of intervention, year of publication or study design (retrospective vs. prospective) were involved in heterogeneity. It should be noted, however, that many other predefined variables (like for example presence of diabetes mellitus and numbers lost to follow up) could not be studied because they were inadequately, if at all, reported. It would therefore be desirable for future pooling of data that authors would conform to reporting standards, such as the guidelines formulated by the American Heart Association. ${ }^{36}$ It is well known that extreme heterogeneity may prevent meaningful conclusions although there is no consensus as to which degree of heterogeneity is still acceptable. By the use of random effects modeling, which allows variation in the underlying effect size between studies to be taken into account, we diminished this effect at least partly. The estimation of the size of the effect, however, should be interpreted cautiously especially since the quality of many studies was rather disappointing. Secondly, from a methodological point of view, it would have been better to compare the change in creatinine between patients (randomly allocated) to active treatment and patients treated with (maximum) conservative therapy in stead of change of serum creatinine in an active treatment group only. However, only three such trials exist. ${ }^{15,16,18} \mathrm{Up}$ to date, two meta-analyses have been published that considered these reports. ${ }^{37,38}$ The study by Nordmann et al. could not pool data with respect to renal function because they were reported in different ways in the original reports. The study by Ives et al. gathered data from the authors and found (by pooling data from the studies by Plouin et al. and Van Jaarsveld et al.) a small advantage of angioplasty over conservative treatment (difference in serum creatinine of $6 \mu \mathrm{mol} / \mathrm{l}$ ). This difference was, however, not statistically significant. The analysis was, however, seriously underpowered since it was based on a comparison between 75 patients who underwent angioplasty and 72 who were treated by drugs only (of which 22 or $31 \%$ had undergone angioplasty within the follow-up period). Although inferior from a methodological point of view, observational studies do have a specific role in medical effectiveness research. Patients enrolled in RCT's often differ from the average patients in clinical practice. Indeed, the setting and the applied intervention in a observational study probably approximate clinical practice better than that in the RCT. In accord with the metaanalysis by Ives et al., ${ }^{38}$ our analysis, showed a small, yet statistically significant, benefit with respect to renal function. 


\section{Conclusion}

This systematic review adds a new piece to the existing evidence suggesting that it is unlikely that intervention will lead to major improvement of renal function. 


\section{References}

1. Caps MT, Perissinotto C, Zierler RE, Polissar NL, Bergelin RO, Tullis MJ, Cantwell-Gab K, Davidson RC, Strandness DE, Jr. Prospective study of atherosclerotic disease progression in the renal artery. Circulation. 1998;98:2866-72.

2. Downs SH, Black N. The feasibility of creating a checklist for the assessment of the methodological quality both of randomised and non-randomised studies of health care interventions. J Epidemiol Community Health. 1998;52:377-84.

3. Lipsey MW, Wilson DB. Selecting, computing and coding the effect size statistic. In: Practical meta-analysis. Thousand Oaks: SAGE Publications, Inc; 2001:34-71.

4. Rosano TG, Brown HH. Analytical and biological variability of serum creatinine and creatinine clearance: implications for clinical interpretation. Clin Chem. 1982;28:2330-1.

5. Pekelharing JM. Handboek klinisch-chemische tests. Utrecht: Bunge; 1995.

6. Canzanello VJ, Millan VG, Spiegel JE, Ponce PS, Kopelman RI, Madias NE. Percutaneous transluminal renal angioplasty in management of atherosclerotic renovascular hypertension: results in 100 patients. Hypertension. 1989;13:163-72.

7. Ziakka S, Belli AM, Kong TK, MacGregor GA, Missouris CG. Percutaneous transluminal renal artery angioplasty: who benefits most? Int J Clin Pract. 2002;56:649-54.

8. van de Ven PJ, Kaatee R, Beutler JJ, Beek FJ, Woittiez AJ, Buskens E, Koomans HA, Mali WP. Arterial stenting and balloon angioplasty in ostial atherosclerotic renovascular disease: a randomised trial. Lancet. 1999;353:282-6.

9. Weibull H, Bergqvist D, Bergentz SE, Jonsson K, Hulthen L, Manhem P. Percutaneous transluminal renal angioplasty versus surgical reconstruction of atherosclerotic renal artery stenosis: a prospective randomized study. J Vasc Surg. 1993;18:841-52.

10. Thompson SG, Sharp SJ. Explaining heterogeneity in meta-analysis: a comparison of methods. Stat Med. 1999;18:2693-708.

11. Greminger P, Steiner A, Schneider E, Kuhlmann U, Steurer J, Siegenthaler W, Vetter W. Cure and improvement of renovascular hypertension after percutaneous transluminal angioplasty of renal artery stenosis. Nephron. 1989;51:362-6.

12. Rodriguez-Perez JC, Plaza C, Reyes R, Pulido-Duque JM, Palop L, Ferral H, Maynar M, Castaneda-Zuniga WR. Treatment of renovascular hypertension with percutaneous transluminal angioplasty: experience in Spain. J Vasc Interv Radiol. 1994;5:101-9.

13. White WB, Mansoor GA, Singh S, Ramsby GS, Ruby ST. Determinants of long-term blood pressure reponse to renal revascularization. J Vasc Med Biol. 1994;5:16-21.

14. Jensen G, Zachrisson BF, Delin K, Volkmann R, Aurell M. Treatment of renovascular hypertension: one year results of renal angioplasty. Kidney Int. 1995;48:1936-45.

15. Plouin PF, Chatellier G, Darne B, Raynaud A. Blood pressure outcome of angioplasty in atherosclerotic renal artery stenosis: a randomized trial. Essai Multicentrique Medicaments vs Angioplastie (EMMA) Study Group. Hypertension. 1998;31:823-9.

16. Webster J, Marshall F, Abdalla M, Dominiczak A, Edwards R, Isles CG, Loose H, Main J, Padfield P, Russell IT, Walker B, Watson M, Wilkinson R. Randomised comparison of percutaneous angioplasty vs continued medical therapy for hypertensive patients with atheromatous renal artery stenosis. Scottish and Newcastle Renal Artery Stenosis Collaborative Group. J Hum Hypertens. 1998;12:329-35.

17. Symonides B, Chodakowska J, Januszewicz A, Lapinski M, Januszewicz M, Rowinski O, Szmidt J, Kuch-Wocial A, Kurzyna M, Malek G, Berent H, Szmigielski C, Januszewicz W. Effects of the correction of renal artery stenosis on blood pressure, renal function and left ventricular morphology. Blood Press. 1999;8:141-50.

18. Van Jaarsveld BC, Krijnen P, Pieterman H, Derkx FH, Deinum J, Postma CT, Dees A, Woittiez AJ, Bartelink AK, Man in 't Veld AJ, Schalekamp MA. The effect of balloon angioplasty on hypertension in atherosclerotic renal-artery stenosis. Dutch Renal Artery Stenosis Intervention Cooperative Study Group. N Engl J Med. 2000;342:1007-14.

19. Bloch MJ, Trost DA, Whitmer J, Pickering TG, Sos TA, August P. Ostial renal artery stent placement in patients 75 years of age or older. Am J Hypertens. 2001;14:983-8. 
20. Kennedy DJ, Colyer WR, Brewster PS, Ankenbrandt M, Burket MW, Nemeth AS, Khuder SA, Thomas WJ, Shapiro JI, Cooper CJ. Renal insufficiency as a predictor of adverse events and mortality after renal artery stent placement. Am J Kidney Dis. 2003;42:926-35.

21. Ramos F, Kotliar C, Alvarez D, Baglivo H, Rafaelle P, Londero H, Sanchez R, Wilcox CS. Renal function and outcome of PTRA and stenting for atherosclerotic renal artery stenosis. Kidney Int. 2003;63:276-82.

22. Coen G, Moscaritolo E, Catalano C, Lavini R, Nofroni I, Ronga G, Sardella D, Zaccaria A, Cianci R. Atherosclerotic renal artery stenosis: one year outcome of total and separate kidney function following stenting. BMC Nephrol. 2004;5:15.

23. Sivamurthy N, Surowiec SM, Culakova E, Rhodes JM, Lee D, Sternbach Y, Waldman DL, Green RM, Davies MG. Divergent outcomes after percutaneous therapy for symptomatic renal artery stenosis. J Vasc Surg. 2004;39:565-74.

24. Bonelli FS, McKusick MA, Textor SC, Kos PB, Stanson AW, Johnson CM, Sheedy PF, 2nd, Welch TJ, Schirger A. Renal artery angioplasty: technical results and clinical outcome in 320 patients. Mayo Clin Proc. 1995;70:1041-52.

25. Blum U, Krumme B, Flugel P, Gabelmann A, Lehnert T, Buitrago-Tellez C, Schollmeyer P, Langer M. Treatment of ostial renal-artery stenoses with vascular endoprostheses after unsuccessful balloon angioplasty. N Eng/ J Med. 1997;336:459-65.

26. Ahmadi R, Schillinger M, Sabeti S, Loewe C, Mlekusch W, Haumer M, Banyai M, Lammer J, Minar E. Renal artery PTA and stent implantation: immediate and late clinical and morphological outcome. Wien Klin Wochenschr. 2002;114:21-7.

27. Soulez G, Therasse E, Qanadli SD, Froment D, Leveille M, Nicolet V, Turpin S, Giroux MF, Guertin MC, Oliva VL. Prediction of clinical response after renal angioplasty: respective value of renal Doppler sonography and scintigraphy. AJR Am J Roentgenol. 2003;181:1029-35.

28. Henry M, Amor M, Henry I, Ethevenot G, Tzvetanov K, Courvoisier A, Mentre B, Chati Z. Stents in the treatment of renal artery stenosis: long-term follow-up. $J$ Endovasc Surg. 1999;6:42-51.

29. Tuttle KR, Chouinard RF, Webber JT, Dahlstrom LR, Short RA, Henneberry KJ, Dunham LA, Raabe RD. Treatment of atherosclerotic ostial renal artery stenosis with the intravascular stent. Am J Kidney Dis. 1998;32:611-22.

30. Rocha-Singh KJ, Mishkel GJ, Katholi RE, Ligon RA, Armbruster JA, McShane KJ, Zeck KJ. Clinical predictors of improved long-term blood pressure control after successful stenting of hypertensive patients with obstructive renal artery atherosclerosis. Catheter Cardiovasc Interv. 1999;47:167-72.

31. Dorros G, Jaff M, Mathiak L, He T. Multicenter Palmaz stent renal artery stenosis revascularization registry report: four-year follow-up of 1,058 successful patients. Catheter Cardiovasc Interv. 2002;55:182-8.

32. Henry M, Henry I, Klonaris C, Polydorou A, Rath P, Lakshmi G, Rajacopal S, Hugel M. Renal angioplasty and stenting under protection: the way for the future? Catheter Cardiovasc Interv. 2003;60:299-312.

33. Zeller T, Frank U, Muller C, Burgelin K, Sinn L, Bestehorn HP, Cook-Bruns N, Neumann FJ. Predictors of improved renal function after percutaneous stent-supported angioplasty of severe atherosclerotic ostial renal artery stenosis. Circulation. 2003;108:2244-9.

34. lannone LA, Underwood PL, Nath A, Tannenbaum MA, Ghali MG, Clevenger LD. Effect of primary balloon expandable renal artery stents on long-term patency, renal function, and blood pressure in hypertensive and renal insufficient patients with renal artery stenosis. Cathet Cardiovasc Diagn. 1996;37:243-50.

35. White CJ, Ramee SR, Collins TJ, Jenkins JS, Escobar A, Shaw D. Renal artery stent placement: utility in lesions difficult to treat with balloon angioplasty. J Am Coll Cardiol. 1997;30:1445-50.

36. Rundback JH, Sacks D, Kent KC, Cooper C, Jones D, Murphy T, Rosenfield K, White C, Bettmann M, Cortell S, Puschett J, Clair D, Cole P. Guidelines for the reporting of renal artery revascularization in clinical trials. American Heart Association. Circulation. 2002;106: 1572-85. 
37. Nordmann AJ, Woo K, Parkes R, Logan AG. Balloon angioplasty or medical therapy for hypertensive patients with atherosclerotic renal artery stenosis? A meta-analysis of randomized controlled trials. Am J Med. 2003;114:44-50.

38. Ives NJ, Wheatley K, Stowe RL, Krijnen P, Plouin PF, van Jaarsveld BC, Gray R. Continuing uncertainty about the value of percutaneous revascularization in atherosclerotic renovascular disease: a meta-analysis of randomized trials. Nephrol Dial Transplant. 2003;18:298-304. 


\section{Chapter 8}

Main findings in perspective 



\section{Main findings in perspective}

The studies in this thesis describe several diagnostic (Chapter 2 and 3), pathophysiological (Chapters 4, 5 and 6) and therapeutic aspects (Chapter 7) of renal artery stenosis. The current chapter links up these different aspects and discusses how our findings add to existing knowledge on the discrimination between patients with and without renal artery stenosis and on the functional significance of renal artery stenosis.

\section{Why should renal artery stenosis be diagnosed?}

Before discussing in greater detail how to diagnose renal artery stenosis, it is necessary to highlight why we should diagnose renal artery stenosis in the first place. Clinically, this is useful only when the management of the condition is beneficial, i.e. when the pros outweigh the cons. Whether this is the case for renal artery stenosis is not beyond dispute. Advantages of timely diagnosing any disease may be that the institution of therapy directed specifically at the cause relieves symptoms or that undesirable consequences are averted. Disadvantages include costs and the possibility of complications related to the diagnostic procedures.

To what extent is the timely diagnosis and treatment of renal artery stenosis beneficial? Fairly seldom, renal artery stenosis may present as hypertensive urgency of emergency or, in case of bilateral (near-) occlusive renal artery disease, acute ("flash") pulmonary edema. In most cases, however, the only signs of renal artery stenosis are systemic hypertension and renal dysfunction. Both are independent risk factors for morbidity and mortality. The effect of mechanical treatment of the stenosis on hard end points like end-stage renal disease and cardiovascular morbidity and mortality, has not been investigated so far. While this information is urgently needed, the conduct of a welldesigned randomised clinical trial with adequate power and follow-up will require an enormous effort. Recently, such a trial (the CORAL study) has been approved and is currently undertaken although its results will not become available in the near future. ${ }^{1}$ With respect to the effect on blood pressure, early reports of percutaneous angioplasty in renal artery stenosis already indicated that the chance of clinical success of revascularisation depends on the etiology of renal artery stenosis. In Caucasians, the abnormality is usually caused by atherosclerosis (75\%) or fibromuscular dysplasia (25\%). Other causes, like Takayasu's arteritis, external compression and radiation injury, comprise less than one percent. The reported cure rates of treatment in patients with fibromuscular dysplasia reaches $50 \%$ and in many other patients blood pressure falls to such an extent that less medication is needed. ${ }^{2}$ In atherosclerosis, however, results are less marked. The effect of renal artery patency restoration in atherosclerotic disease on blood pressure and renal dysfunction has been compared to non-specific therapy (use of antihypertensive drugs) in three randomised trials. ${ }^{3-5}$ Meta-analysis of these studies found that patients who underwent revascularisation had a slightly lower 
blood pressure and somewhat reduced antihypertensive drug requirement as compared to patients who were treated conservatively. No effect was found on renal function. ${ }^{6,7}$ These trials have been criticized for non-negligible cross-over between groups and the fact that they did not apply stents, that may decrease the change of restenosis. ${ }^{3-5}$ Apart from these criticisms, the individual randomised clinical trials (and the meta-analyses) had a short follow-up and probably did not have enough power to draw conclusions with respect to renal function. Currently, a randomised clinical trial is being conducted in order to elucidate this issue, but it will take some time before its results will be disclosed. $^{8}$ Therefore, we performed a meta-analysis (Chapter 7 ) of all available evidence the results of which suggests that with the current practice of patient selection it is unlikely that intervention will lead to major improvement of renal function within one to two years after intervention. However, it was not possible to evaluate long-term effects. It seems too early, therefore, to give a definite answer to the question whether revascularisation therapy can avert undesirable (long term) consequences.

Pursuing a diagnosis of renal artery stenosis may also provide disadvantages. Renal angiography, which is still the gold standard for diagnosis, is expensive amounting $€ 1721$ according to a recent calculation. ${ }^{9}$ More importantly, it brings about a (small) chance of potentially serious complications like atheromatous embolization of the kidneys, renal artery dissection, radiocontrast-induced nephropathy, retroperitoneal bleeding, femoral pseudoaneurysm and hematoma requiring surgical exploration. The chance of such serious complications is low and depends also on underlying morbidity. In the RADISH study, which compared computed tomographic angiography (CTA) and magnetic resonance angiography (MRA) to conventional digital subtraction angiography (DSA), the rate of serious adverse events was $0.7 \%$ (three out of 402 patients, one died within 30 days after DSA, one allergic reaction to contrast agent and one dissection of the renal artery necessitating nephrectomy). ${ }^{10}$

The question thus remains whether it is worthwhile to diagnose renal artery stenosis. This question is of course difficult to answer in general, because it depends also on the clinical context, like the number of functioning kidneys or structure and function of the poststenotic kidney(s). Yet, in our opinion, with the current state of knowledge, the answer should be a cautious "yes". First of all, not diagnosing renal artery stenosis would imply that also patients with FMD, who have a fair chance of cure, are denied causative therapy. As described in Table 1.1 in Chapter 1, these patients cannot be readily distinguished from patients with atherosclerotic disease on the basis of simple characteristics like age and sex. Secondly, in patients with atherosclerotic disease revascularisation may lead, on average, to a modest fall in blood pressure and some reduction in medication use. The effect on long-term renal outcome, however, is not yet known. The effect of this slight improvement in blood pressure and reduced need for medication, may be greater than suggested by absolute numbers. It is a well known phenomenon that results from clinical trials should be translated cautiously to every day 
practice. Clinical trials tend to be executed in specialized institutions. Such institutions may treat a highly selected group of patients that may contain more unusual or difficult "cases" who may have different a priori chances of success. On the other hand, the achievements of interventional radiologists (or vascular surgeons) in these centres may be better compared to those in routine clinical practice. Participants in clinical trials are under tighter control than would normally occur in general care. Further, consenting patients may have different values with respect to health than refusers. ${ }^{11}$ In which direction the sum of these factors will influence treatment outcomes outside trials, is hard to tell. Presumably, both the tighter control and the restriction of the evaluation of outcome to patients who consented to participation in a clinical trial, lead to a better compliance with therapy in clinical trials than in every day practice. The effect of revascularisation is compliance-independent, whereas the use of drugs is obviously not. Adherence to treatment declines with the number of prescribed pills. ${ }^{12,13}$ It may therefore very well be that in general practice the reduced need for antihypertensive drugs after mechanical treatment results in greater differences in blood pressure between patients treated with revascularisation and those conservatively treated than in clinical trials. This may be of importance since in the prevention of cardiovascular disease and renal failure, millimetres do count. ${ }^{14,15}$ Of course, before advocating an invasive treatment solely on the basis of this argument, patient preferences, alternative strategies (for example, the promotion of therapy-adherence and/or lifestyle modification) and cost-effectiveness considerations will have to be taken into account. Thirdly, revascularisation may have beneficial effects on vascular and renal function through mechanisms other than blood pressure reduction or renal function salvage. In studies investigating split renal function in unilateral disease, revascularisation reversed the state of hypoperfusion through the poststenotic kidney and the hyperperfusion through the kidney contralateral to the stensosis without any change in average kidney function. ${ }^{3,16-18}$ Furthermore, endothelial function may improve after angioplasty. ${ }^{19}$ Provided that these changes prove to be long-lasting, they may affect long-term outcome.

\section{How to identify patients with renal artery stenosis?}

Usually, suspicion of renal artery stenosis is raised by the presence of severe hypertension in association with one or more clinical clues. ${ }^{20,21}$ When there is a high degree of suspicion, renal angiography may be performed. During the past decade, most research concerning the diagnosis of renal artery stenosis has focused on the development of accurate non-invasive diagnostic tests to be used in patients with some degree of clinical suspicion of renal artery stenosis. Initial reports regarding the value of MRA, CTA and renal duplex ultrasonography for this purpose ranged from positive to very enthusiastic. ${ }^{22}$ As a consequence, consensus guidelines had already qualified MRA, CTA and renal duplex as acceptable screening tests before evidence from welldesigned trials became available. ${ }^{23,24}$ To our knowledge, only one large prospective 
observer-blinded study compared MRA and CTA to the gold standard, and found that these tests do not live up to their expectations and should not be used, neither as diagnostic tests nor as screening tests. This conclusion is based primarily on the low sensitivity of the diagnostic modalities (CTA 64\%, MRA 62\%); specificity was adequate (CTA 92\%, MRA 84\%). ${ }^{10}$

Based on the disappointing performance of non-invasive tests, we studied whether the efficiency of the use of renal angiography for the diagnosis of renal artery stenosis could be improved on the basis of clinical clues and risk factors easily obtainable through anamnesis, physical examination or simple diagnostic tests. (Chapter 2) To this end, we assessed the prevalence of clinical clues and risk factors for cardiovascular disease in general in patients who underwent renal angiography because of a high clinical suspicion. This restricts the validity of our findings to patients who present with similar conditions, i.e. patients between 18 and 75 years of age with persistent hypertension and with at least one clinical clue ${ }^{a}$ who are referred to secondary/tertiary care centres. In this group of patients, the prevalence of renal artery stenosis was $20 \%$. Of all clues and risk factors, only a higher age, a high pulse pressure, recent onset of hypertension (within the two years before referral), smoking, widespread atherosclerosis and a length difference between the kidneys independently predicted the presence of renal artery stenosis. Four out of the six predictors (age, pulse pressure, smoking and atherosclerosis elsewhere) are among the usual clues for any manifestation of atherosclerosis, which is in the majority of patients the cause of the disease under study. The other two predictors seem to be more related to the specific features of renovascular hypertension (more sudden onset of hypertension) and consequences (reduction of renal volume). When these factors are known, the probability of renal artery stenosis can be predicted through summation of the scores associated with the presence (and the magnitude) of the predictors. This probability may be used as a guide to whom one should refer for angiography. One may decide to perform angiography only when the probability of renal artery stenosis is higher than a certain threshold value. For example, when a threshold value of $10 \%$ is chosen, sensitivity and specificity for the detection of stenosis are $93 \%$ and $38 \%$ respectively. Would this rule have been applied to our cohort, the number of angiographies could have been reduced by $38 \%$. More efficient use of renal angiography may lead to a reduction in costs, and possibly also absolute complication rate. Inevitably, some patients with renal artery stenosis are

\footnotetext{
${ }^{a}$ persistent hypertension despite (at least) two antihypertensive drugs or diastolic blood pressure $>120 \mathrm{mmHg}$ on single-drug therapy, malignant or accelerated hypertension, sudden onset or worsening of hypertension, severe hypertension before the age of 30 or after the age of 60 , unexplained (or sudden onset of ) renal dysfunction, angiotensin converting enzyme (ACE)-inhibitor induced renal function deterioration, renal bruit, unilateral small kidney, extensive (atherosclerotic) occlusive disease, suspicion of restenosis after previously successfully treated renal artery lesions, smoking, high serum cholesterol concentration, low serum potassium concentration, and/or left ventricular hypertrophy
} 
missed when using this cut-off value. Would the rule have been applied to our cohort, $11 \%$ of the stenosis would have been missed. Whether this is a major disadvantage, depends on whether this concerns patients who would benefit from specific treatment, which is currently unknown. Two published models proposing a diagnostic strategy as to whom should be subjected to angiography, namely the prediction rule from the DRASTIC study ${ }^{25}$ and the Mann-Pickering strategy, ${ }^{21}$ are commonly referred to. Our model differs from these two in several respects. The application of the DRASTIC rule is restricted to patients with therapy resistant hypertension (defined in that study as hypertension despite the use of at least one drug in patients under the age of forty and at least two drugs above the age of forty) and/or patients with ACE-inhibitor induced renal function deterioration. We believe that clinicians are inclined to suspect renal artery stenosis in broader categories of patients with hypertension. The implementation of the Mann-Pickering strategy is hampered by the need for high quality screening tests which are, as discussed previously, currently unavailable. In this respect, our model may be better fitted for daily practice in the physicians office than previous models although we do acknowledge that our model still has to be validated prospectively. Such evaluation should preferably also include the benefit of the diagnostic strategy. In other words: how many of the patients in whom renal artery stenosis is detected by the prediction model (and confirmed by angiography) benefit from the diagnosis (through a favourable response to revascularisation), and how does this relate to the number of patients who benefited from angiography but were not detected by the prediction model.

Before proceeding to external validation, we wanted to test whether the diagnostic performance could be further improved. Firstly, we evaluated whether the measurement of renal lengths was indispensable for accurate prediction. In order to obtain this information, additional testing, namely renal ultrasound examination, has to be performed with associated costs. Ultrasound data, however, did not improve the model substantially, because this test was usually only abnormal (i.e. a length difference between the kidneys was found) in patients already labelled as high-risk on the basis of the other five predictors. Secondly, we tested whether replacing rather crude measurements of predictors by their more refined counterparts would improve diagnostic accuracy. (Chapter 3 ) We substituted the number of vascular beds with overt atherosclerotic involvement (as assessed by anamnesis, medical history and physical examination) for IMT of the common carotid artery and/or PWV. It has been shown repeatedly that both IMT and PWV are associated with cardiovascular disease and traditional risk factors. ${ }^{26-30}$ Office pulse pressure (as calculated from the three measurements of systolic and diastolic blood pressure during a visit to the outpatient clinic) was replaced by ambulatory measurements (ABP) of pulse pressure (PP) during 24 hours, which have higher reproducibility than office blood pressure measurements and predict cardiovascular risk over and above conventional blood pressure measurements. ${ }^{31}$ Each of the newly considered predictors was related to renal artery 
stenosis, and they were also interrelated. Somewhat to our surprise, however, none of these more sophisticated measurements improved the predictive accuracy of the model.

Our findings indicate that in a pre-selected population with an a priori risk of $20 \%$, it is feasible to detect patients with a low change $(<10 \%)$ of renal artery stenosis on the basis of easily obtainable predictors, provided that our model will prove to be valid in other populations. These patients may then be exempted from angiography. Further improvements in diagnosing renal artery stenosis may be obtained if a screening test test with good test characteristics would become available. Specifically, a low negative likelihood ratio is essential, because this will affect the decision (not) to perform angiography, by decreasing (post-test) probability of renal artery stenosis in case of a negative test result. A high positive likelihood ratio, on the other hand, increases (in the event of a positive screening test) the (post-test) chance of renal artery stenosis. Yet, assuming that that our model is valid, the selection of patients by our model may lead to a pre-(screening)test chance that may be as high as $30 \%$. Since angiography will presumably also be performed in case of $30 \%$ chance of renal artery stenosis, the use of such screening test, which further increases the chance of renal artery stenosis, will not affect the decision to perform angiography,

\section{The functional significance of atherosclertic renal artery stenosis}

One of the most intriguing and still unresolved issues in the pathophysiology of atherosclerotic renal artery stenosis is why even advanced disease does not always have clinical consequences. In experimental models of renovascular hypertension, the relationship between renal artery stenosis and hypertension is straightforward. Narrowing of the renal artery beyond a certain threshold leads to fall in blood flow through the kidney. This is associated with increased renin secretion, which, via the effector hormone angiotensin II leads to relative preservation of the glomerular filtration rate in the affected kidney, abeit, at the cost of high blood pressure. ${ }^{32,33}$ In humans, the relationships between atherosclerotic renal artery stenosis, hypertension and renal dysfunction are complex. Renal artery stenosis may occur alone, or in association with either hypertension or renal dysfunction, or both. ${ }^{34}$ Consequently, the mere fact that a renal artery stenosis is detected in a patient with hypertension does not automatically mean that that person is suffering from renovascular hypertension. Several scenarios may pertain: the renal artery stenosis may cause and sustain hypertension, or the patient may have been suffering from essential hypertension and on top of that a renal artery stenosis has developed. In fact, it is even more complicated: long-standing hypertension irrespective of its cause leads to secondary changes which may sustain hypertension even when the causative factor is removed. The same line of reasoning applies to renal dysfunction and leads to diametrically opposed opinions on the significance of renal artery stenosis. Based on the fact that many patients reaching endstage renal disease with renal artery stenosis have no (other) apparent explanation for 
renal failure, some authors argue that it is likely that renal artery stenosis accounts for $14-20 \%$ of the patients entering dialysis programs. ${ }^{35}$ Others say that it is an innocent bystander with little effect on renal function since in retrospective series remarkably few patients with incidentally detected renal artery stenosis seem to progress to advanced renal failure. ${ }^{36,37}$ With these considerations as a starting point, we will now evaluate the pathophysiological aspects that we studied and reported in this thesis. In experimental models, activation of the renin-angiotensin system and reduction of renal blood flow are cornerstones in the pathophysiology of renovascular hypertension. In the search for an explanation why renal artery stenosis seems to lack functional consequences in a sizable proportion of patients, we decided to concentrate on these aspects in humans.

In the study presented in chapter 4 , we were interested in genetic aspects. Besides the renin-angiotensin system, also endothelial derived nitric oxide may be important in renal blood flow regulation in patients with renal artery stenosis ${ }^{38}$ and therefore we investigated polymorphisms in genes from the renin-angiotensin system (angiotensinogen Met235Thr (AGT M235T), the angiotensin converting enzyme insertion/deletion (ACE I/D) and the angiotensin II type 1 receptor $A 1166 C\left(A T_{1} R\right.$ $\mathrm{A} 1166 \mathrm{C}$ ) polymorphisms) and from the endothelial nitric oxide synthase enzyme (eNOS Glu298Asp polymorphism). Polymorphisms are frequently occurring variations in the human genome. By definition, the frequency of the allele with the lowest prevalence is higher than $1 \%$, but the occurrence rate may vary between populations. In chapter 4 we report an association between the Asp allele of the eNOS Glu298Asp polymorphism with atherosclerotic artery disease. However, the prevalence of this allele differed also significantly between patients with patent renal arteries and both normotensive and hypertensive controls. Therefore, it is likely that the Asp-allele predisposes not so much to renovascular disease in particular, but more to systemic atherosclerosis. Association studies, like the one we performed, may provide evidence of a relationship between a polymorphism and a disease. This (statistical) relationship can be brought upon by three situations. First, the allele itself directly affects the expression of the phenotype. Second, the allele is in linkage disequilibrium with another allele (in the same gene or one at close distance) that is responsible for the effect. Third, the association is brought about by confounding or selection bias. The fact that the association was also apparent in the two control groups, makes the third situation unlikely. Our results do not allow to differentiate between the first and the second situation. Nevertheless, considering the importance of endothelium-derived nitric oxide in atherosclerosis, our findings fit very well in the theoretical framework of atherosclerotic disease and merit further research into the mechanisms whereby altered nitric oxide production in conjunction with other cardiovascular risk factors may cause systemic atherosclerosis. The polymorphisms in genes of the renin-angiotensin system that we studied, were not associated with renal artery disease. Although our findings may provide valuable insights into the pathophysiology of systemic atherosclerosis, the investigated genetic variations do not seem to be involved in pathophysiology of renal artherosclerotic disease per se. 
In order to assess whether renal blood flow studies and key-players of the reninangiotensin system are involved in determining the functional consequences of renal artery disease, we must first be informed about the normal situation. In other words, the situation in patients without any renal artery abnormalities, must be known. Preferably, we would have liked to investigate young, healthy, normotensive patients. However, in order to assess renal blood flow and hormonal activity per kidney, selective arterial and venous catherisation will have to be performed. Obviously, it is not ethical to subject patients without any suspicion of renal artery stenosis to the risks of such an invasive procedure. The second best control group, consisting of patients with hypertension without any renal artery abnormalities, was investigated in chapter 5 . We showed that differences in renal blood flow between the two kidneys were common but unrelated to the level of activity of the renin-angiotensin system. The implicit assumption underlying some diagnostic tests for renal artery stenosis, like renal scintigraphy, is that, in the absence of a hemodynamically significant renal artery stenosis, blood flow through the kidneys will be roughly symmetrical. Indeed, we found that the presence of a clear-cut difference of renal blood flow between the kidneys was associated with a false-positive result of renal scintigraphy and this may provide a partial explanation for the poor predictive ability of renal scintigraphy for the diagnosis of renal artery stenosis. In chapter 6 we examined selective renal blood flow in patients with unilateral atherosclerotic disease. In these patients, at least in the ones with high grade disease, asymmetry was expected. Strikingly, we found that the prevalence of asymmetry in these patients was virtually similar to that in patients without any renal artery abnormalities. Further, in patients with asymmetry, the activity of the renin-angiotensin system was higher, both in patients with low and high grade disease. Both studies in patients with and without renal artery stenosis indicate that the relationship between (grade of) stenosis in the renal artery, renal blood flow and activity of renin angiotensin system is more complex than hitherto assumed. Only in patients with renal artery stenosis, asymmetry was associated with increased activity of the renin angiotensin system. Possibly, this indicates that the determinants of asymmetry are, at least in part, dissimilar. The great majority of patients with high grade renal artery stenosis underwent revascularisation. Only in patients with symmetry of MRBF pulse pressure dropped whereas the decrease in mean arterial pressure after revascularisation was similar in the two groups. Since pulse pressure reflects arterial stiffness, this may indicate that in patients with asymmetry structural changes in the arterial vasculature may prevent changes in pulse pressure. It is probable that in atherosclerotic renal artery disease, structural and/or functional changes in the microvasculature are present. Whereas revascularisation may eliminate arterial luminal reduction, it is less likely that this will affect the pathophysiological conditions in smaller vessels.

From a pathogenetic point of view atherosclerotic renal artery stenosis and fibromuscualar dysplastia represent entirely different diseases. Whereas atherosclerosis 
affects both large and small vessels, FMD is considered to affect only large arteries. FMD may, therefore, be a "better model" for the development of renovascular hypertension (and renal dysfunction) than atherosclerotic renal artery stenosis. The prevalence of FMD, on the other hand, is lower. When an adequately sized group of patients with FMD is assembled in the Maastricht angiography cohort, renal blood flow studies in these patients might shed new light on the issue of asymmetry of renal blood flow.

At the present time, the distinction between functional and non-functional stenoses does not seem to be possible. With this consideration in mind, it may be argued that we should aim at detecting only those patients with renal artery stenosis who have the potential to benefit from treatment. Research into this issue is complicated by the fact that revascularisation sometimes fails because of technical reasons, restenosis may interfere with the outcomes under study and the diagnosis may sometimes be reached at a time when secondary changes have already diminished the chance of a favourable outcome. While detecting reversible renovascular disease does not seem feasible, the reverse, i.e. identifying factors that preclude benefit in terms of blood pressure and renal function from therapy, may be a more achievable goal. Renal length less than nine centimetres and a (bilateral) high resistance index may be among such factors. ${ }^{39,40}$ Future research will have to determine whether the presence of asymmetry of renal blood flow may be added to this list. The routine examination of these factors in a way easily applicable in clinical practice, may further reduce the number of patients subjected to angiography, while not precluding the chance of clinical benefit in others.

The landscape of atherosclerotic renal artery disease has markedly changed over the past decade due to the new imaging modalities and improvements of revascularisation techniques. In spite of this, or perhaps because of this, the discussion on the optimal management causes much controversy among clinicians. This continuing dispute can only be settled by research into appropriate diagnostic and therapeutic strategies, and the studies described in this thesis may have added to this. 


\section{References}

1. Cooper CJ, Murphy TP, Matsumoto A, Steffes M, Cohen DJ, Jaff M, Kuntz R, Jamerson K, Reid D, Rosenfield K, Rundback J, D'Agostino R, Henrich W, Dworkin L. Stent revascularization for the prevention of cardiovascular and renal events among patients with renal artery stenosis and systolic hypertension: rationale and design of the CORAL trial. $\mathrm{Am}$ Heart J. 2006;152:59-66.

2. Ramsay LE, Waller PC. Blood pressure response to percutaneous transluminal angioplasty for renovascular hypertension: an overview of published series. Bmj. 1990;300:569-72.

3. Van Jaarsveld BC, Krijnen P, Pieterman H, Derkx FH, Deinum J, Postma CT, Dees A, Woittiez AJ, Bartelink AK, Man in 't Veld AJ, Schalekamp MA. The effect of balloon angioplasty on hypertension in atherosclerotic renal-artery stenosis. Dutch Renal Artery Stenosis Intervention Cooperative Study Group. N Eng/ J Med. 2000;342:1007-14.

4. Plouin PF, Chatellier G, Darne B, Raynaud A. Blood pressure outcome of angioplasty in atherosclerotic renal artery stenosis: a randomized trial. Essai Multicentrique Medicaments vs Angioplastie (EMMA) Study Group. Hypertension. 1998;31:823-9.

5. Webster J, Marshall F, Abdalla M, Dominiczak A, Edwards R, Isles CG, Loose H, Main J, Padfield P, Russell IT, Walker B, Watson M, Wilkinson R. Randomised comparison of percutaneous angioplasty vs continued medical therapy for hypertensive patients with atheromatous renal artery stenosis. Scottish and Newcastle Renal Artery Stenosis Collaborative Group. J Hum Hypertens. 1998;12:329-35.

6. Nordmann AJ, Woo K, Parkes R, Logan AG. Balloon angioplasty or medical therapy for hypertensive patients with atherosclerotic renal artery stenosis? A meta-analysis of randomized controlled trials. Am J Med. 2003;114:44-50.

7. Ives NJ, Wheatley K, Stowe RL, Krijnen P, Plouin PF, van Jaarsveld BC, Gray R. Continuing uncertainty about the value of percutaneous revascularization in atherosclerotic renovascular disease: a meta-analysis of randomized trials. Nephrol Dial Transplant. 2003;18:298-304.

8. Bax L, Mali WP, Buskens E, Koomans HA, Beutler JJ, Braam B, Beek FJ, Rabelink TJ, Postma CT, Huysmans FT, Deinum J, Thien T, Schultze Kool LJ, Woittiez AJ, Kouwenberg JJ, van den Meiracker AH, Pattynama PM, van de Ven PJ, Vroegindeweij D, Doorenbos CJ, Aarts JC, Kroon AA, de Leeuw PW, de Haan MW, van Engelshoven JM, Rutten MJ, van Montfrans GA, Reekers JA, Plouin PF, La Batide Alanore A, Azizi M, Raynaud A, Harden PN, Cowling M. The benefit of STent placement and blood pressure and lipid-lowering for the prevention of progression of renal dysfunction caused by Atherosclerotic ostial stenosis of the Renal artery. The STAR-study: rationale and study design. J Nephrol. 2003;16:807-12.

9. van Helvoort-Postulart D, Dirksen CD, Kroon AA, Nelemans PJ, de Leeuw PW, Kessels AG, van Engelshoven JM, Hunink MG. Cost analysis of procedures related to the management of renal artery stenosis from various perspectives. Eur Radiol. 2006;16: 154-60.

10. Vasbinder GB, Nelemans PJ, Kessels AG, Kroon AA, Maki JH, Leiner T, Beek FJ, Korst MB, Flobbe K, de Haan MW, van Zwam WH, Postma CT, Hunink MG, de Leeuw PW, van Engelshoven JM. Accuracy of computed tomographic angiography and magnetic resonance angiography for diagnosing renal artery stenosis. Ann Intern Med. 2004;141:674-82.

11. Pocock SJ. Clinical trials: a practical approach. Chichester: Wiley \& Sons; 1983.

12. Eisen SA, Miller DK, Woodward RS, Spitznagel E, Przybeck TR. The effect of prescribed daily dose frequency on patient medication compliance. Arch Intern Med. 1990;150:1881-4.

13. Wetzels GE, Nelemans P, Schouten JS, Prins MH. Facts and fiction of poor compliance as a cause of inadequate blood pressure control: a systematic review. J Hypertens. 2004;22: 1849-55.

14. Staessen JA, Wang JG, Thijs L. Cardiovascular protection and blood pressure reduction: a meta-analysis. Lancet. 2001;358:1305-15.

15. Sarnak MJ, Greene T, Wang X, Beck G, Kusek JW, Collins AJ, Levey AS. The effect of a lower target blood pressure on the progression of kidney disease: long-term follow-up of the modification of diet in renal disease study. Ann Intern Med. 2005;142:342-51. 
16. Mounier-Vehier C, Cocheteux B, Haulon S, Devos P, Lions C, Gautier C, Carre A, Beregi JP. Changes in renal blood flow reserve after angioplasty of renal artery stenosis in hypertensive patients. Kidney Int. 2004;65:245-50.

17. La Batide-Alanore A, Azizi M, Froissart M, Raynaud A, Plouin PF. Split renal function outcome after renal angioplasty in patients with unilateral renal artery stenosis. J Am Soc Nephrol. 2001;12:1235-41.

18. Leertouwer TC, Derkx FH, Pattynama PM, Deinum J, Van Dijk LC, Schalekamp MA. Functional effects of renal artery stent placement on treated and contralateral kidneys. Kidney Int. 2002;62:574-9.

19. Higashi Y, Sasaki S, Nakagawa K, Matsuura H, Oshima T, Chayama K. Endothelial function and oxidative stress in renovascular hypertension. N Engl J Med. 2002;346:1954-62.

20. Detection, evaluation, and treatment of renovascular hypertension. Final report. Working Group on Renovascular Hypertension. Arch Intern Med. 1987;147:820-9.

21. Mann SJ, Pickering TG. Detection of renovascular hypertension. State of the art: 1992. Ann Intern Med. 1992;117:845-53.

22. Vasbinder GBC, Nelemans PJ, Kessels AG, Kroon AA, De Leeuw PW, Van Engelshoven JM. Diagnostic tests for renal artery stenosis in patients suspected of having renovascular hypertension: a meta-analysis. Ann Intern Med. 2001;135:401-11.

23. Rundback JH, Sacks D, Kent KC, Cooper C, Jones D, Murphy T, Rosenfield K, White C, Bettmann M, Cortell S, Puschett J, Clair D, Cole P. Guidelines for the reporting of renal artery revascularization in clinical trials. American Heart Association. Circulation. 2002;106:1572-85.

24. 2003 European Society of Hypertension-European Society of Cardiology guidelines for the management of arterial hypertension. J Hypertens. 2003;21:1011-53.

25. Krijnen P, Van Jaarsveld BC, Steyerberg EW, Man in 't Veld AJ, Schalekamp MA, Habbema JD. A clinical prediction rule for renal artery stenosis. Ann Intern Med. 1998;129:705-11.

26. O'Leary DH, Polak JF, Kronmal RA, Manolio TA, Burke GL, Wolfson SK, Jr. Carotid-artery intima and media thickness as a risk factor for myocardial infarction and stroke in older adults. Cardiovascular Health Study Collaborative Research Group. N Engl J Med. 1999; 340:14-22.

27. Raitakari OT, Juonala M, Kahonen M, Taittonen L, Laitinen T, Maki-Torkko N, Jarvisalo MJ, Uhari M, Jokinen E, Ronnemaa T, Akerblom HK, Viikari JS. Cardiovascular risk factors in childhood and carotid artery intima-media thickness in adulthood: the Cardiovascular Risk in Young Finns Study. Jama. 2003;290:2277-2283.

28. Laurent S, Boutouyrie P, Asmar R, Gautier I, Laloux B, Guize L, Ducimetiere P, Benetos A. Aortic stiffness is an independent predictor of all-cause and cardiovascular mortality in hypertensive patients. Hypertension. 2001;37:1236-41.

29. Blacher J, Asmar R, Djane S, London GM, Safar ME. Aortic pulse wave velocity as a marker of cardiovascular risk in hypertensive patients. Hypertension. 1999;33:1111-7.

30. Simons PC, Algra A, Bots ML, Grobbee DE, van der Graaf Y. Common carotid intima-media thickness and arterial stiffness: indicators of cardiovascular risk in high-risk patients. The SMART Study (Second Manifestations of ARTerial disease). Circulation. 1999;100:951-7.

31. Staessen JA, Thijs L, Fagard R, O'Brien ET, Clement D, de Leeuw PW, Mancia G, Nachev C, Palatini P, Parati G, Tuomilehto J, Webster J. Predicting cardiovascular risk using conventional vs ambulatory blood pressure in older patients with systolic hypertension. Systolic Hypertension in Europe Trial Investigators. Jama. 1999;282:539-46.

32. Anderson WP, Ramsey DE, Takata M. Development of hypertension from unilateral renal artery stenosis in conscious dogs. Hypertension. 1990;16:441-51.

33. Imanishi M, Akabane S, Takamiya M, Kawamura M, Matsushima Y, Kuramochi M, Omae T. Critical degree of renal arterial stenosis that causes hypertension in dogs. Angiology. 1992;43:833-42.

34. Safian RD, Textor SC. Renal-artery stenosis. N Engl J Med. 2001;344:431-42.

35. Scoble JE. The epidemiology and clinical manifestations of atherosclerotic renal disease. In: Novick AC, Scoble JE, Hamilton G, eds. Renal Vascular Disease. London: WB Saunders Co Ltd.; 1996:303-14. 
36. Chabova V, Schirger A, Stanson AW, McKusick MA, Textor SC. Outcomes of atherosclerotic renal artery stenosis managed without revascularization. Mayo Clin Proc. 2000;75:437-44.

37. Leertouwer TC, Pattynama PM, van den Berg-Huysmans A. Incidental renal artery stenosis in peripheral vascular disease: a case for treatment? Kidney Int. 2001;59:1480-3.

38. Wierema TK, Houben AJ, Kroon AA, Koster D, van der Zander K, van Engelshoven JM, de Leeuw PW. Nitric oxide dependence of renal blood flow in patients with renal artery stenosis. J Am Soc Nephrol. 2001;12:1836-43.

39. Radermacher J, Chavan A, Bleck J, Vitzthum A, Stoess B, Gebel MJ, Galanski M, Koch KM, Haller $\mathrm{H}$. Use of Doppler ultrasonography to predict the outcome of therapy for renal-artery stenosis. N Engl J Med. 2001;344:410-7.

40. Soulez G, Therasse E, Qanadli SD, Froment D, Leveille M, Nicolet V, Turpin S, Giroux MF, Guertin MC, Oliva VL. Prediction of clinical response after renal angioplasty: respective value of renal Doppler sonography and scintigraphy. AJR Am J Roentgenol. 2003;181:1029-35. 


\section{Chapter 9}

Summary 



\section{Summary}

Renovascular hypertension is considered to be the second most common cause of secondary hypertension. About one percent of all individuals with elevated blood pressure in the general population has a stenosis in one or more of their renal arteries. The presence of renal artery stenosis may lead to renovascular hypertension and renal dysfunction. Restoration of renal artery patency may, at least potentially, reverse hypertension and prevent (further) deterioration of kidney function. The relatively high prevalence of renal artery stenosis contrasts with our rather poor understanding of the interrelationships between renal artery lumen reduction, renal perfusion pressure and renal blood flow on the one hand and their alleged consequences hypertension and renal dysfunction, on the other. The studies that form the main body of this thesis investigated diagnostic ((chapter 2 and 3), pathophysiological (chapters 4, 5 and 6) and therapeutic (chapter 7) aspects of renal artery stenosis. These studies were designed and conducted to assess whether these aspects could 1) distinguish patients with renal artery stenosis from with moderate to severe hypertension without such a lesion and 2) shed light on the pathophysiology of renal artery stenosis and/or renovascular hypertension. The current chapter summarizes main findings from these studies.

In chapter 2, we assessed the predictive power of established clinical clues ${ }^{1,2}$ and traditional cardiovascular risk factors for the diagnosis of renal artery stenosis in our cohort. We found that the majority of clinical clues had only limited predictive value. Six predictors were identified: age, pulse pressure, current or past smoking behaviour, onset of hypertension within two years, extra-renal atherosclerosis and a difference in kidney lengths. A model incorporating these six predictors achieved an optimismcorrected area under the receiver-operating characteristic curve of 0.75 . Hence, even though the value of established clinical clues for prediction of renal artery stenosis in common clinical practice is low, the number of patients undergoing renal angiography may be reduced with our model. Of course, before the use of our model can be recommended for clinical practice, the model should be validated in other population(s).

In chapter 3, we investigated the hypothesis that the precision of predictions by the model presented in chapter 2 could be increased by replacing crude measurements of the predictors pulse pressure and extra-renal atherosclerosis by more refined counterparts (24-hour ambulatory pulse pressure and intima-media thickness (IMT) of the common carotid artery and/or aortic pulse wave velocity (PWV) respectively). Each of the newly considered predictors was related to renal artery stenosis, and they were also interrelated. Somewhat to our surprise, however, none of these more sophisticated measurements improved the predictive accuracy of the model. Our results, with respect to PWV and IMT, corroborate findings in populations at high risk as well as at low risk with respect to various manifestations of cardiovascular disease. IMT and PWV seem to 
have no additional value in the prediction of cardiovascular disease over and above easily obtainable traditional risk factors.

In chapter 4, we report a study on the association of four polymorphisms in candidate genes with atherosclerotic renal artery disease. It concerns the angiotensinogen Met235Thr, the angiotensin converting enzyme insertion/deletion, the angiotensin II type 1 receptor A1166C and the eNOS Glu298Asp polymorphisms. In this chapter, normotensive and hypertensive patients without a history of cardiovascular disease from a family practice served as external controls. No differences were found with respect to the polymorhisms in genes of the renin-angiotensin system. The Asp-allele of the endothelial nitric oxide synthase Glu298Asp polymorphism on the other hand, was associated with atherosclerotic renal artery stenosis with an odds ratio (95\% confidence interval) of 1.44 (1.00-2.09) versus hypertensives with angiographically-proven patent arteries, of 1.89 (1.24-2.87) versus hypertensive family practice controls and of 2.09 (1.29-3.38) versus normotensive family practice controls. However, this allele differed also significantly between patients with patent renal arteries and both normotensive and hypertensive controls. Since atherosclerotic renovascular disease is generally viewed upon as a local manifestation of systemic atherosclerotic disease our data support earlier observations in this respect. The absence of atherosclerotic lesions in renal arteries, namely, does not at all exclude the presence of atherosclerosis elsewhere in the vascular system and, indeed, nearly a quarter of the patients whose arteries turned out to be patent (after angiography) had experienced signs of extrarenal atherosclerosis. Moreover, it is likely that normotensive and hypertensive patients in a general care practice have lower amounts of (extra-renal) atherosclerosis than patients in an outpatient clinic of a secondary/tertiary referral hospital who are suspected of having renal artery stenosis. Thus, we hypothesize that the Asp-allele of the eNOS Glu298Asp polymorphism predisposes to (systemic) atherosclerosis.

In chapter 5, we studied selective renal blood flow in the patients in our cohort without any renal artery abnormality. We found clear-cut left-right differences ("asymmetry") in $51 \%$ of the patients. Subjects with and without asymmetry did not differ in age, body mass index, blood pressure, creatinine clearance, renal volume or activity of the reninangiotensin system. Preliminary data suggest that there may be a relation between asymmetry and renal sympathetic nerve activity, which might point to the fact that the differences may be, at least partially, functional. The presence of asymmetry coincided with an increased rate of false-positive results on renal scintigraphy, a screening test for renal artery stenosis that is largely abandoned nowadays because of unfavourable test characteristics. The fact that asymmetry of renal blood flow is a frequent finding in severe hypertension underscores the importance of studying the function of both kidneys separately, since it cannot be assumed that they are functionally equal. 
In chapter 6, we assessed selective renal blood flow in patients with unilateral atherosclerotic renal artery disease of varying severity (15-100\% stenosis). Higher grades of luminal reduction coincided with an increased difference in mean renal blood flow (MRBF) between the stenosed kidney and the contralateral kidney. However, in half of the patients with high grade stenosis, flow through the kidneys was similar or even higher in the kidney with the stenosis than in the contralateral one. Further, patients with asymmetry had greater activity of the renin-angiotensin system regardless of the degree of stenosis. The great majority of patients with high grade disease underwent revascularisation. The decrease in mean arterial pressure after this procedure was similar in both groups, but only in patients with symmetry this was accompanied by a reduction of pulse pressure.

In chapter 7, we performed a meta-analysis of all available evidence on the effect of revascularisation on intermediate renal outcome as measured by a change of creatinine. Review of 29 published series, with a total of 3701 subjects, indicated that although average change of creatinine after intervention differed greatly between the individual studies, renal patency restoration in patients with atherosclerotic renal artery stenosis and hypertension may lead to a small, yet statistically significant, benefit with respect to renal function. It is however unlikely that intervention will lead to major improvement of renal function.

In chapter 8 the results of the studies mentioned above are discussed and put in perspective. 


\section{References}

1. Detection, evaluation, and treatment of renovascular hypertension. Final report. Working Group on Renovascular Hypertension. Arch Intern Med. 1987;147:820-9.

2. Mann SJ, Pickering TG. Detection of renovascular hypertension. State of the art: 1992. Ann Intern Med. 1992;117:845-53. 


\section{Chapter 10}

Samenvatting 



\section{Samenvatting}

Van alle personen met hypertensie ('hoge bloeddruk') in de algemene bevolking heeft ongeveer één procent een stenose ('vernauwing') in een of meer van hun nierarteriën ('nierslagaderen'). De aanwezigheid van zo'n nierarteriestenose kan leiden tot (renovasculaire) hypertensie en vermindering van de nierfunctie. Het is mogelijk de doorgankelijkheid van de aangedane nierarterie te herstellen, met als doel de bloeddrukregulatie te verbeteren en te voorkomen dat de nierfunctie (verder) verslechtert.

De relatief hoge prevalentie van nierarteriestenose staat in scherp contrast met de hiaten in onze kennis van enerzijds de relaties tussen afname van het lumen van de nierarterie, de perfusiedruk en de nierdoorbloeding en anderzijds de uiteindelijke consequenties, te weten hypertensie en afname van de nierfunctie. De studies welke in dit proefschrift worden beschreven, bestudeerden diagnostische (hoofdstukken 2 en 3), pathofysiologische (hoofdstukken 4, 5 en 6) en therapeutische (hoofdstuk 7) aspecten van nierarteriestenose. Deze studies werden opgezet en uitgevoerd om te weten te komen of met behulp van deze aspecten: 1 . in een ziekenhuispopulatie van patiënten met matig tot ernstige hypertensie een onderscheid kan worden gemaakt tussen degenen met een nierarteriestenose en degenen die deze afwijking niet hebben en 2. meer inzicht verkregen kan worden in de pathofysiologie van nierarteriestenose en renovasculaire hypertensie.

In het tweede hoofdstuk wordt de studie beschreven waarin de waarde van veel gebruikte klinische kenmerken ${ }^{1,2}$ en de traditionele cardiovasculaire risicofactoren werd bepaald bij het voorspellen van het vóórkomen van nierarteriestenose. De analyses lieten zien dat de overgrote meerderheid van deze wijd aanvaarde klinische kenmerken nagenoeg geen voorspellende waarde hadden. Het vóórkomen van nierarteriestenose bleek wel samen te hangen met de volgende zes variabelen: leeftijd, polsdruk, roken of in het verleden gerookt hebben, recent (<2 jaar) ontstaan van hypertensie, atherosclerose in andere vaatbedden en een verschil in lengte tussen de twee nieren. Een model met deze zes variabelen behaalde een gecorrigeerde oppervlakte onder de receiver-operating characteristic curve van 0.75 . Aldus lijkt het met behulp van dit model mogelijk een redelijke uitspraak te doen over welke patiënten een zeer lage kans hebben op een nierarteriestenose en bij gebruik van het model kan het aantal patiënten dat een nierangiografie moet ondergaan sterk gereduceerd worden. Echter, voordat dit model in de praktijk gebruikt kan worden, moet het gevalideerd worden in andere populaties.

In het derde hoofdstuk hebben we getracht het model dat gepresenteerd werd in hoofdstuk 2 te verbeteren door verfijndere technieken te gebruiken om de polsdruk en extra-renale atherosclerose te kwantificeren. Hiervoor gebruikten we respectievelijk 
ambulante bloeddrukregistratie en metingen van intima-media dikte (IMT; a. carotis communis) en pulse wave velocity (PWV; traject a. carotis communis - a. femoralis). Alhoewel deze metingen gerelateerd waren aan het vóórkomen van nierarteriestenose, verbeterden ze de voorspellingen van het model uit hoofdstuk twee niet. Dit is in lijn met recente studies waarin IMT en PWV cardiovasculaire ziekten niet beter lijken te voorspellen dan veel eenvoudiger te bepalen traditionele risicofactoren.

In hoofdstuk 4 werd de associatie tussen nierarteriestenose op basis van atherosclerose en vier genetische polymorfismen in kandidaat-genen getoetst. Het betroffen polymorfismen in de angiotensinogeen (Met235Thr), the angiotensin converting enzyme (insertion/deletion), the angiotensine II type 1 receptor (A1166C) and the eNOS (Glu298Asp) genen. Normotensieve en hypertensieve patiënten uit een huisartspraktijk dienden als extra controle groepen. Er werd geen associatie gevonden tussen de bestudeerde polymorfismen in de genen van het renine-angiotensine systeem. Het Asp-allel van het eNOS Glu298Asp polymorfisme was geassocieerd met atherosclerotische nierarteriestenose: met een odds ratio $(95 \%$ betrouwbaarheidsinterval) van 1.44 (1.00-2.09) versus patiënten met hypertensie en middels angiografie bewezen gave arteriën, van 1.89 (1.24-2.87) versus patiënten met hypertensie in een huisartspraktijk en van 2.09 (1.29-3.38) versus patiënten zonder hypertensie in een huisartspraktijk. Echter, dit allel was eveneens significant vaker aanwezig bij patiënten met hypertensie en middels angiografie bewezen gave arteriën dan bij patiënten in de huisartspraktijk (zowel met als zonder hypertensie). Een kwart van de patiënten welke een nierangiografie ondergingen en geen nierarteriestenose bleken te hebben had elders in het lichaam uitingen van atherosclerose. Mogelijk is het Asp-allel of the eNOS Glu298Asp polymorfisme niet zo zeer specifiek geassocieerd met atherosclerotische nierarteriestenose als wel met atherosclerose sec.

In het vijfde hoofdstuk bestudeerden we de nierdoorbloeding van beide nieren afzonderlijk bij patiënten in het angiografiecohort zonder afwijkingen van de nierarteriën. Bij $51 \%$ van deze patiënten vonden we duidelijke verschillen (asymmetrie) tussen de twee nieren. Patiënten met en zonder asymmetrie verschilden niet in leeftijd, body mass index, bloeddruk, geschatte creatinine klaring, niervolume of de mate van activiteit van het renine-angiotensine systeem. Mogelijk is er wel een relatie tussen asymmetrie en renale sympaticus activiteit, en dit is een aanwijzing dat deze verschillen deels of geheel functioneel zouden kunnen zijn. Patiënten met een asymmetrische nierdoorbloeding hadden vaker een vals-positieve uitkomst bij nierscintigrafie, een screeningstest welke op basis van onvoldoende diagnostische nauwkeurigheid weinig meer gebruikt wordt. Het frequente voorkomen van aanzienlijke verschillen in nierdoorbloeding tussen de twee nieren onderstreept het belang van het bestuderen van beide nieren afzonderlijk aangezien niet op voorhand aangenomen kan worden dat ze vanuit functioneel opzicht gelijk zijn. 
In hoofdstuk 6 werd selectief de nierdoorbloeding gemeten bij patiënten met een unilaterale atherosclerotische nierarteriestenose, welke in ernst varieerde tussen de 15 en $100 \%$. Hieruit bleek dat de mate van stenosering een duidelijke relatie had met het voorkomen van asymmetrie in nierdoorbloeding tussen de twee nieren. Echter, patiënten met een ernstige nierarteriestenose hadden niet vaker asymmetrie in nierdoordoorbloeding dan patiënten zonder nierarteriestenose. Opmerkelijk was dat bij ongeveer de helft van de patiënten met een ernstige stenose er een betere doorbloeding was van de nier met de stenose dan de nier zonder de stenose. De overgrote meerderheid van patiënten met een ernstige stenose onderging revascularisatie. Hoewel bij patiënten met symmetrie en asymmetrie een gelijke daling van de gemiddelde bloeddruk gezien werd, daalde alleen bij patiënten met symmetrie ook de polsdruk.

Hoofdstuk 7 beschrijft een meta-analyse van 29 studies (met in totaal 3701 patiënten) naar het effect van revascularisatie bij atherosclerotische nierarteriestenose op de nierfunctie op de middellange termijn. Hieruit concludeerden we dat het niet waarschijnlijk is dat revascularisatie zal leiden tot een belangrijke verbetering van de nierfunctie.

De bovengenoemde studies worden in perspectief geplaatst in hoofdstuk 8. 


\section{References}

1. Detection, evaluation, and treatment of renovascular hypertension. Final report. Working Group on Renovascular Hypertension. Arch Intern Med. 1987;147:820-9.

2. Mann SJ, Pickering TG. Detection of renovascular hypertension. State of the art: 1992. Ann Intern Med. 1992;117:845-53. 
Dankwoord 



\section{Dankwoord}

Als $u$ op deze pagina bent beland door alle voorgaande pagina's te lezen zult $u$ begrijpen dat ik ze onmogelijk allemaal heb kunnen schrijven zonder de hulp en inspiratie van anderen. Vele personen hebben bijgedragen aan de totstandkoming van dit proefschrift. Sommigen heel concreet. Anderen, niet minder essentieel, door regelmatig belangstelling te tonen voor het onderzoek, verhalen aan te horen, kopjes koffie in te schenken op momenten dat het even allemaal tegenzat, en met name in de laatste fase, vooral ook niet te vaak te vragen naar de vorderingen. Allen bedankt! Een aantal personen wil ik graag in het bijzonder noemen.

Allereerst alle patiënten die belangeloos meewerkten aan de beschreven onderzoeken.

Prof. dr. Peter de Leeuw, ruim acht jaar geleden klopte ik bij je aan met de vraag of er misschien projecten waren waar ik mijn wetenschapstage op zinvolle wijze aan zou kunnen besteden. Met enthousiasme vertelde je me over de uiteenlopende projecten waar nog talloze onderzoeksvragen lagen te wachten. Dit enthousiasme bleek later kenmerkend voor je te zijn, en hiermee, in combinatie met je enorme kennis van de literatuur en (patho)fysiologie, wist je me voortdurend te motiveren nieuwe wegen in te slaan wanneer ik weer eens even vast zat. Ik bewaar goede herinneringen aan de schrijfweken in Vals, niet alleen vanuit wetenschappelijk oogpunt zeer effectief, maar ook een leuke manier om je eens van een andere, niet-wetenschappelijke kant te leren kennen.

Dr. Boy Houben, jij hebt vanaf mijn start als frisse wetenschaps-co de dagelijkse begeleiding op je genomen. Nadat je me eerst de basis van enkele basisvaardigheden hebt moeten aanleren ("Kijk, dit is een computer en met deze knop gaat hij aan") gingen we voortvarend aan de slag. De resultaten van deze stage hebben geleid tot hoofdstuk 5 en een presentatie in de grote congreszaal in Milaan. Als bijwerking merkte ik hoe leuk ik het doen van onderzoek bleek te vinden en jouw enthousiaste wijze van begeleiding heeft daar een belangrijke rol in gespeeld. Als co-promotor bleef je nauw betrokken bij mijn onderzoeken en was je vaak degene die het gemakkelijkst te bereiken was voor overleg. Graag wil ik jou ook bedanken voor de gezelligheid tijdens de congressen en andere bijeenkomsten, al zal ik je padvindersinstinct node missen.

Dr. Bram Kroon, nadat duidelijk werd dat de gestarte onderzoeken in mijn wetenschapsstage uitgebouwd zouden gaan worden tot een promotieonderzoek, kwam jij als co-promotor het "promotieteam" versterken. De clinicus in je zorgde dat de klinische relevantie van het onderzoek, waar nodig, bewaakt werd. Ik heb het gewaardeerd dat je me de ruimte en de vrijheid gaf mijn eigen plannen en ideeën te volgen. 
Claudia de Haan, Monique Fuss-Lejeune en Dorien Bleeker wil ik bedanken voor hun inzet en het meedenken bij het uitvoeren van de diverse onderzoeken.

Wanneer een proefschrift zowel "diagnostische strategieën" als "nierarteriestenose" in de titel heeft, is het duidelijk dat dit alleen tot stand kan komen door veel inspanningen op radiologisch vlak. Ik wil de afdeling radiologie van het azM, en in het bijzonder Prof dr. Jos van Engelshoven, drs. Derk Koster, dr. Kiam Oei, dr Boudewijn Vasbinder en dr. Michiel de Haan van harte bedanken voor de goede samenwerking.

Drs. ir. Fons Kessels, dr. Patty Nelemans en dr. Karin Flobbe wil ik bedanken voor de vele constructieve methodologische en statistische discussies die we voerden gedurende het ontwikkelen van het predictiemodel uit hoofdstuk 2. De data welke gebruikt werden als controlegroepen in hoofdstuk 5 werden beschikbaar gesteld door de onderzoekers van de HIPPOCRATES studie, en in het bijzonder drs. Léon Henskens, drs. Arian Plat en dr. Henri Stoffers. Dr. Maurice Zeegers en Dr. Marinus van Kroonenburgh leverden essentiële bijdragen aan respectievelijk hoofdstuk 5 en 6.

De leden van de beoordelingscommissie, prof dr. J.M.A van Engelshoven, prof dr. P.J.E.H.M. Kitslaar, prof dr. H.A. Koomans en prof. dr. M.H. Prins, wil ik van harte danken voor het kritisch beoordelen van mijn manuscript. I wish to thank prof dr. P.F. Plouin for his willingness to review my thesis.

Er waren congressen, schrijfweken, kerstdiners, vele kopjes koffie al dan niet met vlaai, etentjes, uitstapjes per step en kano, methodologische- en computerproblemen, feesten: collega's van "'t circulatielab" Esther Beeks, Jörgen ten Berge, Claudia Beyrus, Dorien Bleeker, Stella Brand, Monique Fuss-Lejeune, Claudia de Haan, Léon Henskens, Marian Hommels, Heidi Jongen, Melanie van der Klauw, Marrigje NabuursFransen, Roger Renneberg, Sergio Rodriguez, Ingrid Scheffers, Wilko Spiering, Suuske Vasbinder, Willem Verberk, Thomas Wierema en Kim van der Zander van harte bedankt voor het delen van wetenschappelijke en overige hoogte-en dieptepunten!

Tiny Wouters, ik wil je heel hartelijk bedanken voor je enorme inzet om mijn boekje er zo mooi uit te laten zien. Ik heb meermaals bewonderend staan kijken dat je in enkele minuten zoveel goeds kon doen voor een figuur waar ik dan al uren aan had gewerkt. Je weet precies wat er allemaal moet gebeuren voor je uiteindelijk voor de corona staat, hetgeen voor mij een hele geruststelling was bij het voorbereiden van de promotie vanaf zekere afstand.

Maaike Vreeburg en Jennifer van Leur, lieve vriendinnen, het geeft me een goed gevoel jullie, ook nu weer, bij dit hoogtepunt, als paranimfen naast me te hebben staan.

Ik wil eindigen met het bedanken van alle mensen die strikt genomen niets met mijn proefschrift te maken, maar wiens steun onmisbaar was. Ach wat, ik vertel het jullie liever persoonlijk... 
Curriculum vitae 



\section{Curriculum vitae}

Marieke van Onna werd geboren op 25 mei 1976 te Sittard. In 1994 behaalde ze haar VWO diploma aan het Liemers College te Zevenaar. Aansluitend begon zij aan de studie Geneeskunde aan de Universiteit Maastricht en zij behaalde het artsexamen in december 2000 (cum laude). Vanaf maart 2001 tot en met april 2005 werkte zij als artsonderzoeker bij de werkgroep algemene interne geneeskunde van het academisch ziekenhuis Maastricht. Tijdens deze periode werd de postinitiële master-opleiding Epidemiologie aan de Vrije Universiteit Medisch Centrum in Amsterdam succesvol afgerond. Per 1 mei 2005 is zij gestart met de opleiding Interne Geneeskunde, de eerste twee jaren werkte zij in de Gelre Ziekenhuizen te Apeldoorn (opleider dr. J.M. Smit en later dr. C.G. Schaar) en de opleiding wordt vervolgd in het Universitair Medische Centrum Utrecht (opleider Prof. dr. E. van der Wall). 